This thesis presents the development of a new human machine interface for controf of exos lesetons vila neuromusculos el etal model driven in real time bs experimental ENGs; and oint positions recorded from the user to predigt oint torgue This predicted oint for que is then us d to give assistance to the user via the ex oskeleton.

Albout the atithon:

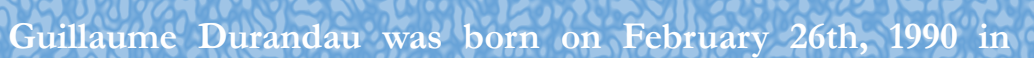

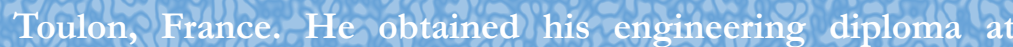

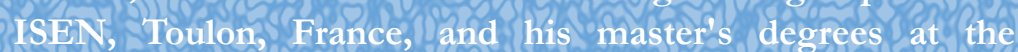

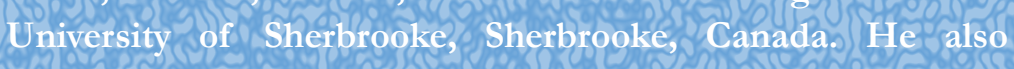
obtained during his master the Mitacs Globalink Research Awards At the beginning of 2014 , he started as a research assistant at the Institute of Neurorehabilitation Systems, UMG, Gottingen Germany under the supervision of Prof 20 is $\mathrm{PhD}$ at the Institute 0

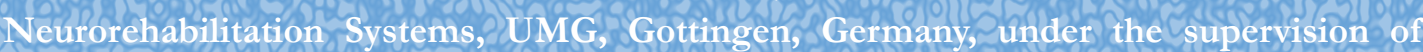
Prof, Dario Farina and D. M assimo saxtorit and then moved in 2014 to the University of Twente, Enschede, the Netherlands to continute his $P$ PD undert the supervision of Prof Herman van der Kooil and Ass of . P rof. Massimo Sartori. During his PhD, he wont the Best Demo Award in 2018 at the 7 th HEEE International Conferences on Biomedica

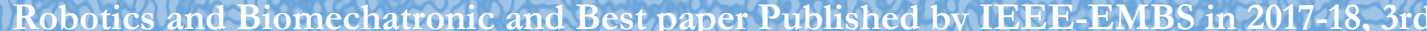
place at the 7 th Dutch Blo-medical Engineering Conference. He is curtently a Posta researcher at the Department of B Bomechanical Engineering fat thes Univerts ity of Ivente, Evischede, the Netherlands 13\%

TSBN: $978-946421,122-1$ DOI: $0.3990 \% 1.9789464211221$

\section{Towards the next Generation}

in Human-machine-interfacing:

Controlling Wearable Robots via

Neuromusculoskeletal Modelling

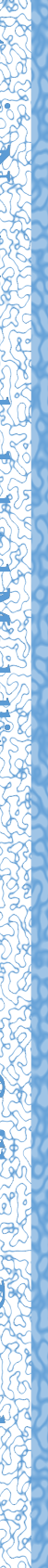

Towards the next Generaton in Human J machine-interfacingt Controlling IKeatable Robots via

Neuromisculos keletal Modelling

Guillaume Durandau

Prof. dr. G. Berkhoff Zaal Waaijer

Friday 6 November 2020 at $10.45 \mathrm{~h}$

Pafanymohs Ronald V an t Veld Simone Fricke

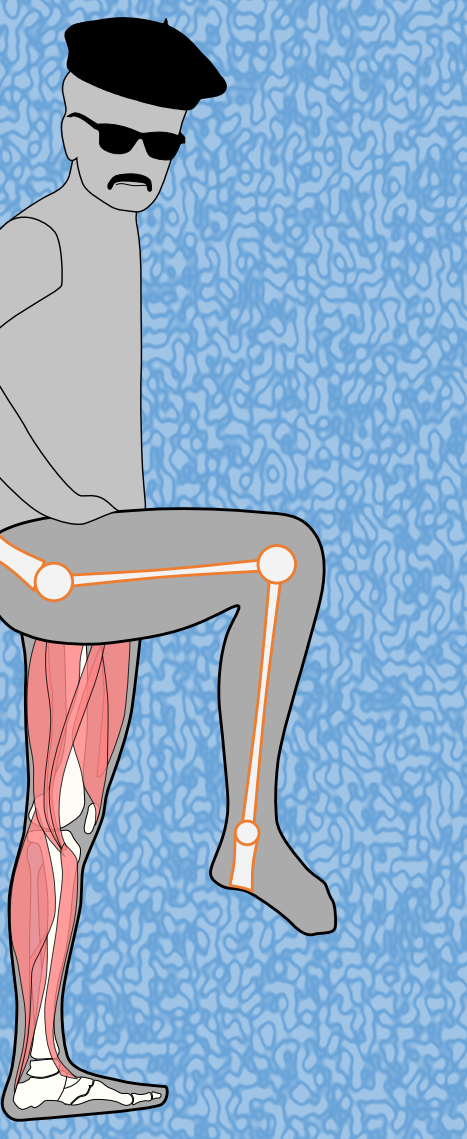





\section{Towards the next Generation}

in Human-machineinterfacing: Controlling Wearable Robots via Neuromusculoskeletal Modelling

Guillaume Durandau 



\title{
Towards the next Generation in Human-machine-interfacing: Controlling Wearable Robots via Neuromusculoskeletal Modelling
}

\author{
DISSERTATION
}

to obtain

the degree of doctor at the Universiteit Twente, on the authority of the rector magnificus,

Prof.dr. T.T.M. Palstra, on account of the decision of the Doctorate Board to be publicly defended on Friday 6 November 2020 at 10.45

by

Guillaume Vincent Durandau

Born the $26^{\text {th }}$ of February 1990 in Toulon, France 


\section{This dissertation has been approved by:}

Promotor: Prof.dr.ir. Herman van der Kooij

Co-promotor \& Supervisor: Dr. Massimo Sartori

Cover design: Ir. Kyrian Staman and Guillaume Durandau

Printed by: Ipskamp Printing

Lay-out: Guillaume Durandau

ISBN: 978-94-6421-122-1

DOI: $10.3990 / 1.9789464211221$

C 2020 Guillaume Vincent Durandau, The Netherlands. All rights reserved. No parts of this thesis may be reproduced, stored in a retrieval system or transmitted in any form or by any means without permission of the author. Alle rechten voorbehouden. Niets uit deze uitgave mag worden vermenigvuldigd, in enige vorm of op enige wijze, zonder voorafgaande schriftelijke toestemming van de auteur. 


\section{Graduation Committee:}

Chairman / Secretary:

Prof.dr.ir. H.F.J.M. Koopman, University of Twente

\section{Promotor:}

Prof.dr.ir. Herman van der Kooij, Department of Biomechanical Engineering, University of Twente and Department of Biomechanical Engineering, Delft University of Technology

\section{Co-promotor \& Supervisor:}

Dr. Massimo Sartori, Department of Biomechanical Engineering, University of Twente

\section{Committee:}

Prof.dr. Dario Farina, Department of Bioengineering, Imperial College London, UK

Dr. Ajay Seth, Department of Biomechanical Engineering, Delft University of Technology

Prof.dr. Jaap van Dieën, department of Human Movement Sciences, VU Amsterdam

Prof.dr.MD Hans (J.S.) Rietman, Department of Biomechanical Engineering, University of Twente

Prof.dr.ir. Gijs (J.M.) Krijnen, Department of Robotics and Mechatronics, University of Twente 



\section{CONTENTS}

Biography 2

$\begin{array}{ll}\text { Awards } & 4\end{array}$

$\begin{array}{ll}\text { Publications } & 4\end{array}$

Peer-reviewed Journal Publications 4

Conference Publications 4

$\begin{array}{ll}\text { Summary } & 6\end{array}$

$\begin{array}{ll}\text { Introduction } & 9\end{array}$

1.1 Motivation 9

1.2 Approach 12

1.3 Goal 14

1.4 Outline of this dissertation 16

Robust Real-Time Musculoskeletal Modelling Driven By Electromyograms 18

2.1 Introduction 20

2.2 Real-time EMG-Driven Modelling 22

2.3 Experimental Procedures 28

2.4 Results 29

2.5 Discussion 32

2.6 Conclusion 34

Toward Muscle-Driven Control of Wearable Robots: a Real-Time Framework for the Estimation of Neuromuscular States During Human-Exoskeleton Locomotion Tasks

3.1 Introduction 39

3.2 Methods 41

3.3 Experiments $\quad 45$

3.4 Results 46 
$\begin{array}{llr}3.5 & \text { Discussion } & 47 \\ 3.6 & \text { Conclusion } & 50\end{array}$

Voluntary Control of Wearable Robotic Exoskeletons by Patients with Paresis via Neuromechanical Modeling 53
4.1 Introduction
55
4.2 Methods
58
4.3 Results
69
4.4 Discussion
75
4.5 Conclusion

Voluntary and Continuous Control of Robotic Exoskeletons during a broad Repertoire of Locomotion Conditions

5.1 Introduction 83

5.2 Methods 84

5.3 Experiment 89

5.4 Results 91

$\begin{array}{lll}5.5 & \text { Discussion } & 94\end{array}$

5.6 Conclusion 96

$\begin{array}{ll}\text { Conclusion } & 98\end{array}$

6.1 Summary of Key Findings 99

6.2 Impact and Relevance of the Scientific Contributions 101

6.3 Limitations and Directions for Future Research 102

6.4 Final Conclusion 104

$\begin{array}{ll}\text { References } & 106\end{array}$

$\begin{array}{ll}\text { Appendix } & 119\end{array}$

7.1 Appendix to Introduction 119

$\begin{array}{lll}7.2 & \text { Appendix to Chapter 2 } & 127\end{array}$

7.3 Appendix to Chapter 3 128 
7.4 Appendix to Chapter 4

7.5 Appendix to chapter 5

Acknowledgements 


\section{B IOGRAPHY}

Guillaume Vincent Durandau was born on February 26 ${ }^{\text {th }}, 1990$ in Toulon, France. He obtained his engineering diploma at ISEN, Toulon, France, and his master's degrees at the University of Sherbrooke, Sherbrooke, Canada. The subject of his master was EMG Processing for Control of Robotic System under the supervision of Prof. Wael Suleiman. During his master's, he spent four months at the CINESTAV, Mexico working on human-robot interaction using IMU and EMG signals. He also obtained during his master, the Mitacs Globalink Research Award. At the beginning of 2014, he

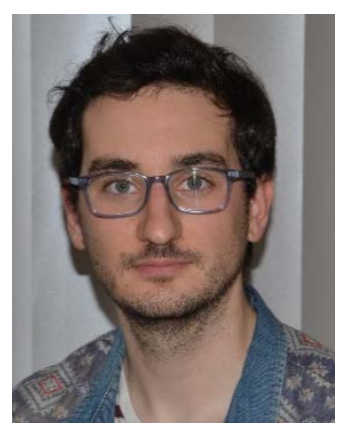
started as a research assistant at the Institute of Neurorehabilitation Systems, UMG, Gottingen, Germany, under the supervision of Prof. Dario Farina and Dr Massimo Sartori. In 2016, he started his $\mathrm{PhD}$ at the Institute of Neurorehabilitation Systems, UMG, Gottingen, Germany, under the supervision of Prof. Dario Farina and Dr Massimo Sartori and then moved in 2017 to the University of Twente, Enschede, the Netherlands to continue his $\mathrm{PhD}$ under the supervision of Prof. Herman van der Kooij and Assoc. Prof. Massimo Sartori. During his PhD, he won the Best Demo Award in 2018 at the 7th IEEE International Conference on Biomedical Robotics and Biomechatronic and Best paper Published by IEEE-EMBS in 2017-18, $3^{\text {rd }}$ place at the $7^{\text {th }}$ Dutch Bio-medical Engineering Conference. He is currently a Postdoc researcher at the Department of Biomechanical Engineering at the University of Twente, Enschede, the Netherlands. 


\section{ORCID}

https://orcid.org/0000-0001-6951$\underline{776 \mathrm{X}}$
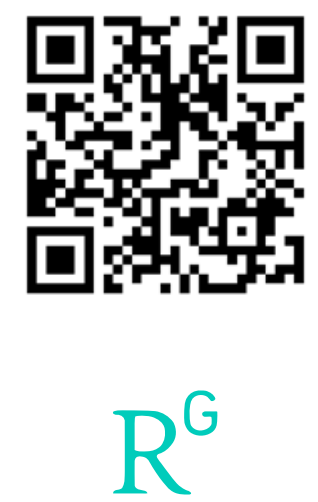

https://www.researchgate.net/profile https://publons.com/researcher/3184 /Guillaume Durandau

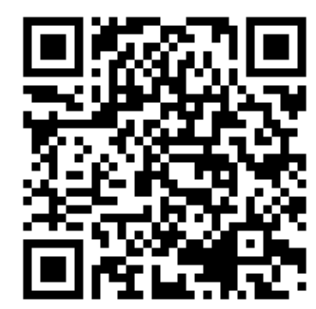

\section{Google Scholar}

https://scholar.google.nl/citations?use $\underline{\mathrm{r}=W u S j V n 0 A A A A J \& h l=\text { en\&oi }=a \mathrm{o}}$
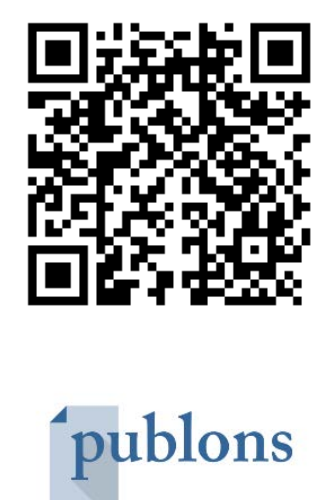

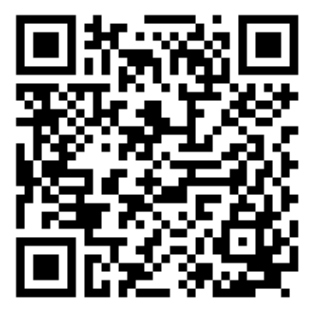




\section{AWARDS}

1. Best paper Published by IEEE-EMBS in $2017-18,3^{\text {rd }}$ place, $7^{\text {th }}$ Dutch Bio-medical Engineering Conference.

2. Best Demo Award, 2018 7th IEEE International Conference on Biomedical Robotics and Biomechatronics (Biorob).

\section{PUBLICATIONS}

\section{Peer-reviewed Journal Publications}

1. Durandau, G., Farina, D., \& Sartori, M. (2017). Robust real-time musculoskeletal modeling driven by electromyograms. IEEE transactions on biomedical engineering, 65(3), 556-564.

2. Sartori, M., Durandau, G., Došen, S., \& Farina, D. (2018). Robust simultaneous myoelectric control of multiple degrees of freedom in wrist-hand prostheses by real-time neuromusculoskeletal modeling. Journal of neural engineering, 15(6), 066026.

3. Durandau, G., Farina, D., Asín-Prieto, G., Dimbwadyo-Terrer, I., Lerma-Lara, S., Pons, J. L., ... \& Sartori, M. (2019). Voluntary control of wearable robotic exoskeletons by patients with paresis via neuromechanical modeling. Journal of neuroengineering and rehabilitation, 16(1), 91.

4. Lotti, N., Xiloyannis, M., Durandau, G., Galofaro, E., Sanguineti, V., \& Sartori, M. (2020). Adaptive model-based myoelectric control for a soft wearable arm exosuit: A new generation of wearable robot control. IEEE Robotics \& Automation Magazine.

\section{Conference Publications}

1. Romanato, M., Sartori, M., Durandau, G., Volpe, D., \& Sawacha, Z. (2019). An EMG-informed modelling approach for the prediction of internal variables during locomotion in Parkinson's disease patients: a feasibility study. Gait \& Posture, 74, 32-33.

2. Esteban, A. M., van't Veld, R. C., Cop, C. P., Durandau, G., Sartori, M., \& Schouten, A. C. (2019, July). Estimation of Time-Varying Ankle Joint Stiffness Under Dynamic Conditions via System Identification Techniques. In 2019 41st Annual International Conference of the IEEE Engineering in Medicine and Biology Society (EMBC) (pp. 2119-2122). IEEE.

3. Cop, C. P., Durandau, G., Esteban, A. M., van't Veld, R. C., Schouten, A. C., \& Sartori, M. (2019, July). Model-based estimation of ankle joint stiffness during dynamic tasks: a validation-based approach. In 201941 st Annual International 
Conference of the IEEE Engineering in Medicine and Biology Society (EMBC) (pp. 41044107). IEEE.

4. Sartori, M., Durandau, G., Dosen, S., \& Farina, D. (2018, October). Decoding phantom limb neuro-mechanical function for a new paradigm of mind-controlled bionic limbs. In International Conference on NeuroRebabilitation (pp. 54-57). Springer, Cham.

5. Sartori, M., Durandau, G., van der Kooij, H., \& Farina, D. (2018, October). Multiscale modelling of the human neuromuscular system for symbiotic human-machine motor interaction. In International Conference on NeuroRebabilitation (pp. 167-170). Springer, Cham.

6. Durandau, G., van der Kooij, H., \& Sartori, M. (2018, October). A computational framework for muscle-level control of bi-lateral robotic ankle exoskeletons. In International Symposium on Wearable Robotics (pp. 325-328). Springer, Cham.

7. Durandau, G., Rampeltshammer, W., Van Der Kooij, H., \& Sartori, M. (2018, August). Toward Muscle-Driven Control of Wearable Robots: A Real-Time Framework for the Estimation of Neuromuscular States During HumanExoskeleton Locomotion Tasks. In 2018 7th IEEE International Conference on Biomedical Robotics and Biomechatronics (Biorob) (pp. 683-688). IEEE.

8. Sartori, M., Durandau, G., \& Farina, D. (2017). Neuromusculoskeletal Models of Human-Machine Interaction in Individuals Wearing Lower Limb Assistive Technologies. In Converging Clinical and Engineering Research on Neurorehabilitation II (pp. 827-831). Springer, Cham.

9. Durandau, G., Sartori, M., Bortole, M., Moreno, J. C., Pons, J. L., \& Farina, D. (2017). Real-time modeling for lower limb exoskeletons. In Wearable Robotics: Challenges and Trends (pp. 127-131). Springer, Cham.

10. Durandau, G., Sartori, M., Bortole, M., Moreno, J. C., Pons, J. L., \& Farina, D. (2016). EMG-driven models of human-machine interaction in individuals wearing the $\mathrm{H} 2$ exoskeleton. IFAC-papersonline, 49(32), 200-203. 


\section{SUMMARY}

To this day, robotic rehabilitation has not met its promise. It did not revolutionize rehabilitation of patients after stroke or spinal cord injury yet. One of the challenges hampering this goal is the control and communication interface between the human and the machine. Currently, commercial exoskeletons replay pre-defined gait patterns, while research exoskeletons replay optimized torque profiles or assist proportionally to electromyograms (EMG) signals. In most cases, the dynamics of the human musculoskeletal system is ignored, simplified or considered as a black-box. This dissertation goal is to endow wearable robots with numerical representations of the human body to enable robust and intuitive human control of wearable robots. To achieve this goal, a change of paradigm in control of wearable robots is proposed in this dissertation, going away form pure robotic control where the human is driven by the robotic device to a new paradigm where the human drives the robotic device.

This dissertation presents the development of a new human-machine interface (HMI) for control of exoskeletons via a neuromusculoskeletal model driven in real-time by experimental EMGs and joint positions recorded from the user to predict joint torques. These predicted joint torques are then used to assist the user via exoskeletons.

First, the development of a real-time version of the HMI previously created by Sartori et al was accomplished by incorporating a B-spline algorithm for real-time computation of the of muscle-tendon lengths and moment arms from joint positions. Furthermore, the computational efficiency of the HMI was increased so that computational time was brought below the muscle electromechanical delay (i.e. $<50 \mathrm{~ms}$ ). Further work was done to create real-time inverse kinematics and inverse dynamics pipelines informed by experimentally recorded marker positions and ground reaction forces. This was tested on five healthy subjects where results showed that the developed HMI could estimate muscle-tendon forces and joint torques online with direct validation against inverse dynamics (gold standard). Results indicated that our HMI could extrapolate across new movements and new degrees of freedom that were not used to calibrate the model.

Secondly, the developed HMI was employed to enable torque control of wearable exoskeletons. Tests were done on stroke and spinal cord injury patients performing seated rehabilitation motor tasks. Results demonstrated that the HMI translated human bioelectrical muscle activity in exoskeleton control commands leading to reductions in EMG amplitudes as well as variability in the patients' group.

Thirdly, further tests were conducted on locomotion tasks with different modalities (speeds and/or elevations) with healthy users. This experiment proved the possibility to assist positively (i.e. reductions of EMGs and biological torques) across different locomotion tasks 
and transitions across tasks. Results showed that the total human + exoskeleton torques were always similar between the exoskeleton assisting mode and when the exoskeleton was in minimal impedance (i.e. transparent mode). This means that force transfers between the human and the exoskeleton were created where the provided assistance was fully integrated by the human thereby lowering biological joint torque levels by the same amount as the received torques from the exoskeleton.

The development of this new HMI offers new possibilities for control of robotic devices as well as opens new avenues in assistive wearable robotics. 


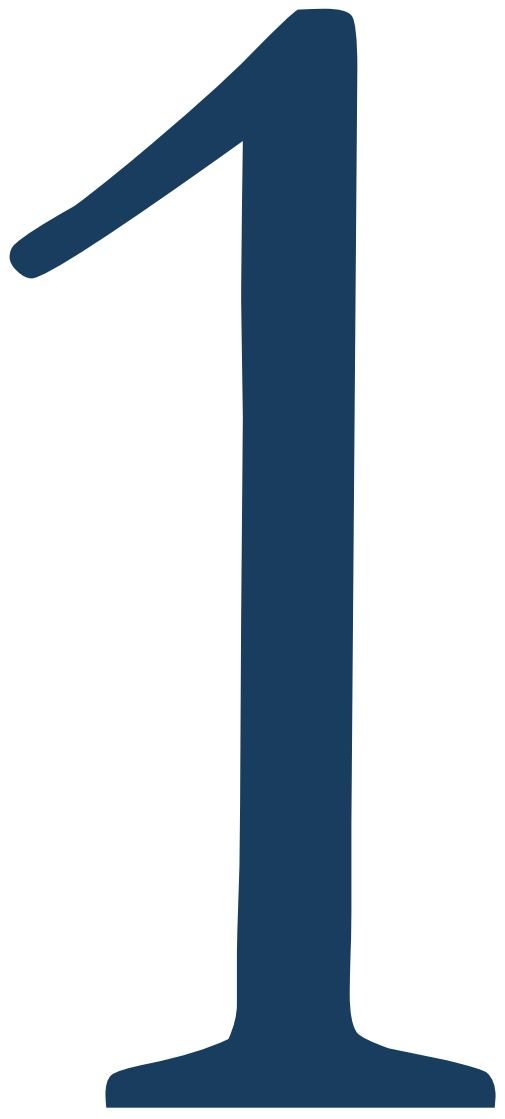




\subsection{Motivation}

Each year 6,3 million people worldwide [1] and 174.000 people in the Netherlands ${ }^{1}$ suffer from a stroke episode with a total of 42,4 million survivors of strokes worldwide. Of those who have a stroke in the USA, 15\% do not survive. From the survival group, only $10 \%$ regain the full functionality and the rest will have to live with some kind of disability ${ }^{2}$. The impact of rehabilitation after stroke is still limited and the success of rehabilitation procedures is mainly dependent on the skill of the medical expert ${ }^{3}$. Furthermore, between 250.000 and 500.000 persons suffer from a spinal cord injury (SCI) worldwide ${ }^{4}$. SCI patients have 2 to 5 times more chances to die prematurely than non-SCI.

Moreover, there are additional causes of motor impairment such as amputation, after a traumatic accident (3\% [2]), oncology linked diseases (3\% [2]) and more importantly, vascular linked diseases (94\% [2]) (for the lower-limb, data for the north of the Netherlands). Unfortunately, only $66 \%$ return to work after amputation for lower-limb and 53\% to $100 \%$ for upper-limb [3]. The return to work after amputation can be halted by the restricted mobility inherent to prostheses [3].

Finally, in factory settings, work-related injuries may lead to musculoskeletal disorders, loss of quality of life and have a negative economic impact. The $12^{\text {th }}$-month prevalence of musculoskeletal disorder ranges from 2.3 to $41 \%$ and the lifetime prevalence can be up to 29\% [4] (for upper-limb).

Neuromusculoskeletal injury's impact on the quality of life could be mitigated by assistive wearable robotic devices. These robotic devices can take the form of exoskeletons, as well as prostheses for amputees and allow to provide forces in parallel (i.e. exoskeletons) or in series (i.e. prostheses) to a joint. For rehabilitation, they can be fully ambulatory like the EksoNR $^{5}$ (Ekso Bionic, USA) or Rewalk Personal ${ }^{6}$ (Rewalk, USA). They can also be nonambulatory with a fixed support base like the Lokomat ${ }^{7}$ (Hocoma, Switzerland). For prostheses, the most common and technologically advanced upper-limb prostheses are the

\footnotetext{
1 https://www.hersenstichting.nl/alles-over-hersenen/hersenaandoeningen/cijfers-overpatienten

2 http://www.stroke.org/we-can-help/stroke-survivors/just-experienced-stroke/rehab

${ }^{3}$ https://www.healthline.com/health/stroke/recovery\#outlook6

4 https://www.who.int/news-room/fact-sheets/detail/spinal-cord-injury

${ }^{5}$ https://eksobionics.com/eksohealth/

${ }^{6}$ https:/ / rewalk.com/rewalk-personal-3/

7 https://www.hocoma.com/solutions/lokomat/
} 
Michelangelo ${ }^{8}$ hands and Bebionic ${ }^{9}$ hand from Ottobock, Germany. For the knee, the Genium ${ }^{10}$ and for the ankle, the empower ${ }^{11}$, are the most advanced lower-limb prostheses also developed by Ottobock. Finally, in an industrial setting, most of the proposed exoskeletons are aimed for the back and are passive like the Laevo V2 ${ }^{12}$ (Laevo, the Netherlands) and the EskoVest ${ }^{13}$ (Ekso Bionic, USA). See part 7.1.1 of the Appendix for a presentation of the current state of the art on the research wearable devices used in this dissertation.

Assistive wearable robotic devices are already in use but suffer from limitations. Assistive robotic devices for rehabilitation allow higher intensity training than classic rehabilitation without robotic devices and demand less manpower from physiotherapists. However, each stroke patient is unique and most of the robotic devices do not account for that. Moreover, rehabilitation relies on the patient's voluntary involvement in the task to activate neuroplasticity, something current robotic devices do not easily enable as the user does not fully voluntarily control them. Furthermore, for prostheses, on average $25 \%$ of myoelectric prostheses users stopped or will stop using their prostheses because their quality of life does not improve [5] (for upper-limb). For the industrial setting, passive exoskeletons severely restrict the number of tasks that can be efficiently assisted as the springs that compose most of these passive devices and their line of action cannot be dynamically changed to adapt to new tasks. Moreover, active exoskeletons could potentially assist a wide variety of tasks but are limited by their controllers.

The aforementioned limitations are due to the human-machine interface (HMI) of assistive robotic devices that cannot adapt to multiple tasks and do not offer voluntary control. HMI represents the connection between the human and the machine via kinematics, kinetics and bio-signals data recorded from the human. It also represents the connection between the machine and the human via the assistance provided.

HMIs did not advance as much as their mechatronic counterpart (i.e., motor, computer power ...) in assistive wearable robotics or as the electrode design and recording techniques (i.e., bio-signal recording, nerve interface ...). For exoskeletons, a major paradigm shift on

\footnotetext{
8https://www.ottobockus.com/prosthetics/upper-limb-prosthetics/solutionoverview/michelangelo-prosthetic-hand/

'https://www.ottobockus.com/prosthetics/upper-limb-prosthetics/solutionoverview/bebionic-hand/

${ }^{10}$ https://www.ottobockus.com/prosthetics/lower-limb-prosthetics/solutionoverview/genium-above-knee-system/

${ }^{11}$ https://www.ottobockus.com/prosthetics/lower-limb-prosthetics/solutionoverview/empower-ankle/

12 https:/ / laevo-exoskeletons.com/laevo-v2

13 https://eksobionics.com/eksoworks/
} 
mechatronic design happened with advances like soft exoskeleton [6] that promises to deliver a lightweight and more discrete exoskeleton (i.e. placed under clothes), and series-elastic actuation [7] that offers better torque or stiffness control. New electrode designs and bioelectrical signal processing like high-density electromyography (EMG) recording [8] allow us to better understand motor control by the estimation of motor neuron activities. Also, surgical advances such as muscle reinnervation surgery [9], allow recording electrical impulses from previously cut nerves (i.e. connecting the missing muscles of the amputated limb). Tactile biofeedback via muscle reinnervation [10] promises to reroute tactile feedback from amputated limbs to cut nerves.

For a long time, researches in HMI for wearable devices have considered the body as a blackbox, where biomechanical processes underlying human movement were ignored or simplified. Namely, the control and design of exoskeletons were mostly based upon mechanical inputs (i.e. mechanical assistance) -outputs (i.e. metabolic consumption, interaction forces between devices and users) of the user and ignored the internal properties of the human body that are influenced by the device. An instance of this black-box problem can be seen in the current state of the art approaches. Pre-recorded gait patterns are used to control exoskeletons for rehabilitation in the state of the art of commercially available devices (i.e., Lokomat, Hocoma, Switzerland). State of the art in exoskeleton control has proposed methods based on impedance control [11], pre-recorded torque pattern [6], and optimized torque pattern [12]. These solutions are limited in terms of flexibility and do not enable support of large sets of motor tasks, i.e. they do not allow other tasks than treadmill walking with the gait constrained in speed and do not allow start and stop of the gait. Moreover, current solutions have only had a modest impact on clinical scenarios involving neurologically impaired individuals. These modest impacts can partially be explained by the black-box paradigm used. In myoelectric prostheses, there are two main types of controllers, a direct EMG controller using two electrodes with one electrode controlling the positive ways (flexion) and the other the negative one (extension) from one degree of freedom (DOF). To change the controlled DOF the user has to produce a muscle co-contraction. The second kind of controller is based on machine learning algorithms, where EMG is used to classify movements. The first method allowed to achieve robust control but requires training of the user and allows control only one DOF at the time. The second method allowed a high level of recognition success $(>90 \%)$ but is limited in the number of tasks or DOFs and is highly sensitive to changes in body-posture [13].

As we have seen, in HMI, kinematics or torque reference pattern based controllers are still in predominance limiting extrapolation of the controllers on untrained tasks. Biological signal-based controls do not investigate internal mechanisms of the human body limiting biomechanical benefices. As an alternative to current research on HMI that considers human as a black-box, a new class of methods using biomechanical modelling can be developed. This method is called neuromusculoskeletal modelling as it allows a biomechanical model 
(i.e., the mechanical part) to estimate the user's intention through EMG processing (i.e., surrogate of neural drive to muscles). This method is based on EMG-driven modelling, which is the virtual representation of the human body (i.e., kinematic and dynamic parameters) [14]. See Part 7.1.2 and 7.1.3 of the Appendix for a presentation of the human muscle physiology and EMG-driven modelling. This method was chosen because it offers to compute a large set of biomechanical outputs such as joint torques, muscle forces [14], joint contact forces [15], and joint stiffnesses [16] with a more wearable set of sensors than classic methods (i.e. inverse dynamics with static optimization). This large set of mechanical outputs is central for better understanding the impact of the wearable (i.e., the exoskeleton) on the wearer (i.e., the user) and thus offering better controllers. It also offers the advantage to be personalized [17] through calibration and scaling, which is of first importance for patients with multiple and different impairments or deficits. Moreover, this method is not bound to any task and does not need any further algorithm to switch between states to adapt to other tasks in contrary to other pre-computed torque or position patterns. Finally, this HMI solution can easily be adapted to other exoskeletons or body parts as only the substitution of the model to a new one needs to be done.

Experiments and results presented in this dissertation were mainly conducted with exoskeletons on the lower-limbs. Nevertheless, as presented in this section, the issues of wearable robotic devices (prostheses and exoskeletons, for upper and lower-limbs) are common between them. The proposed HMI was tested on an upper-limb prosthesis [18] and an upper-limb soft exosuit [19] presenting the same biomechanical benefits as shown in this dissertation.

\subsection{Approach}

All along with this dissertation, the main tool for the developed HMI is a neuromusculoskeletal model, which is driven by EMG also referred to as EMG-driven modelling. This method is based on the Hill-type muscle model which is a numerical representation of the actual muscle, done by A. V. Hill [20] (See part 7.1.3.1 from the Appendix for the equations). In Fig. 1-1, a schematic representation of the developed HMI is shown. From the user, two signals were recorded, EMG signals and joint positions (Fig. 1-1, in grey). These signals went first into an input stage (Fig. 1-1, in red) where they were filtered to remove artefacts and noises. The EMGs were further processed by normalization against the maximum voluntary contractions recorded offline. The filtered joint positions were sent to a surrogates stage (Fig. 1-1, in green) where muscle-tendon lengths and moment arms of the muscles were computed using cubic B-splines algorithm [21]. The muscle activations obtained after normalization of the EMGs and the muscle-tendon lengths were send to a musculo-tendon dynamics stage (Fig. 1-1, in blue) where muscle forces were computed. This stage is based on the Hill-type muscle model and previous works from Lloyd et al. [22] and Sartori et al. [14]. The muscle forces and moment arms were sent to a moment 


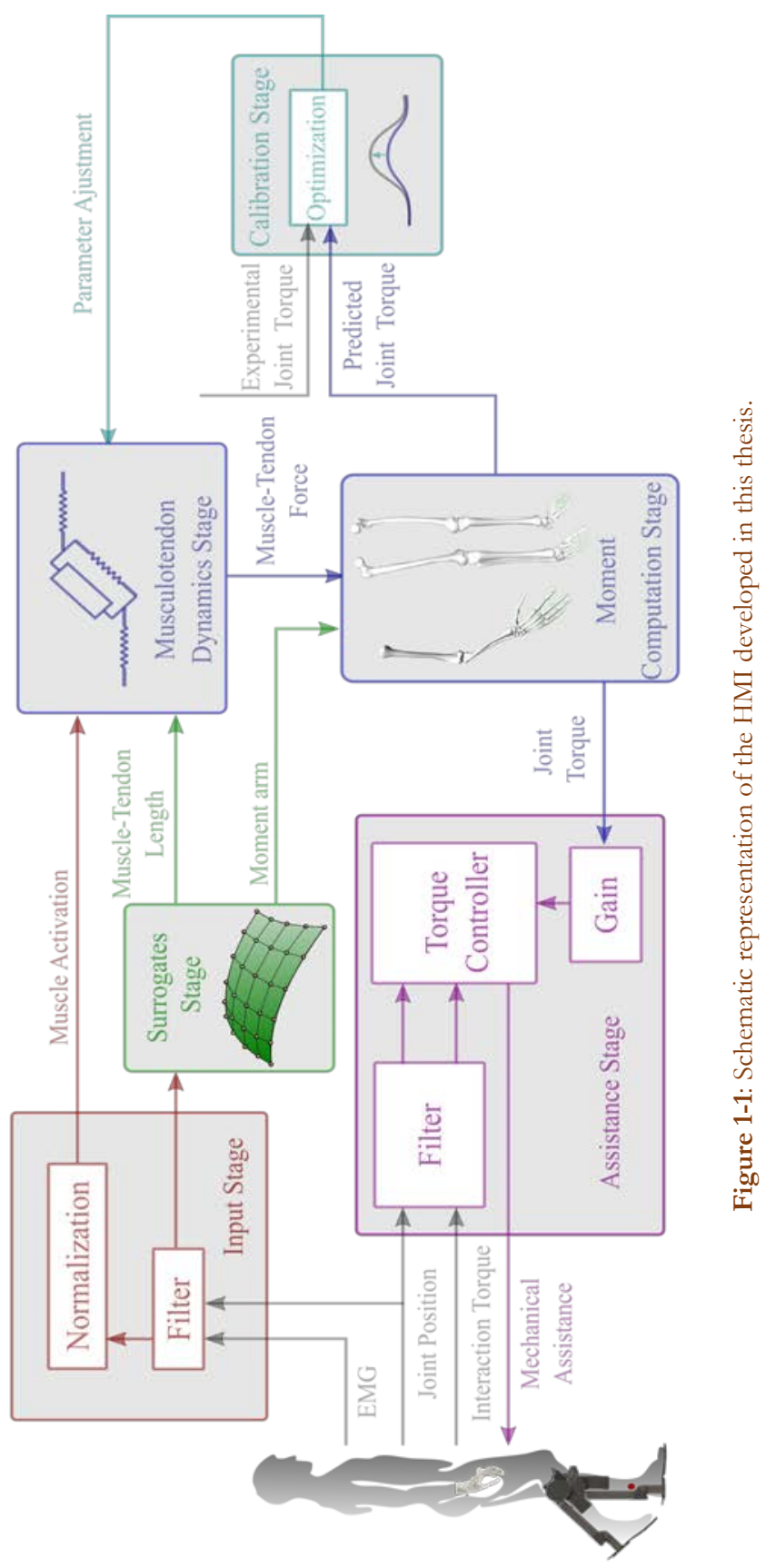


computation stage (Fig. 1-1, in blue) where the joint torques were computed (see section 7.1.3.2 of the Appendix for the method used). The computed joint torques were sent to an assistance stage (Fig. 1-1, in purple) where they were used to control an assistive device like an exoskeleton or a prosthesis device. To obtain assistance, the joint torques were multiplied by a gain (from $10 \%$ to $70 \%$ for an exoskeleton and from $80 \%$ to $120 \%$ for a prosthesis). The assistance was delivered to the users via the device using a torque controller (see section 7.1.1.2 of the Appendix). The neuromusculoskeletal model needs to be personalized to the user to obtain precise joint torque predictions. For this, a calibration stage was used (Fig. 11 , in cyan) to calibrate different muscle parameters such as maximal isometric force, tendon slack length, optimal fiber length, and EMG-to-activation shape factor [14]. An optimization procedure was used, which minimized the error between predicted joint torques and experimental joint torques by changing these parameters [14].

Such HMI has been used already to control orthoses for the arm [23] and the knee [24]. These are interesting proof of concepts but are still limited to one joint [24] or were not directly used to control a device [23]. In the next section, we are presenting the challenges still present for using an EMG-driven model as HMI for the control of assistive wearable robotic devices and we formulate the research questions linked to these challenges.

\subsection{Goal}

The main goal of this dissertation was to create and test a new HMI for lower-limb exoskeletons based on EMG-driven modelling.

To achieve this goal, this dissertation focused on creating a new class of HMI that gives knowledge of the human internal properties to the wearable robot controller. For the estimation of joint torques, the current gold-standard is inverse dynamics which computes joint torques using joint positions and ground reaction forces (GRF) [25]. One of the main issues of this method is that to record GRF, a force plate is needed, which is not a practicable solution when using a wearable robot. This is mostly due to the size of the force plates (only one step for each force plate) and the non-portability of them due to their weight. Another issue is that the user has to be able to produce enough force to create movements to be detected by inverse dynamics, which can be challenging for some patients. Another solution to compute joint torque would be to use machine learning but this approach suffers from extrapolation issues outside of the training data [26]. That is why EMG-driven modelling was chosen in this dissertation for computing joint torque as it offers full portability, only EMG and joint position are needed and offers accurate joint torque computation [14]. Unfortunately, there is currently limited research [23], [24], [27], [28] that bring EMG-driven modelling close to real-time performance (i.e. computation time below the electromechanical delay (EMD)) with a good trade-off between complexity and computation time for multiple degrees of freedom. Furthermore, its extrapolation capabilities, which are keys to possible exploitation with wearables devices are unknown. 
From this, we have deducted the following research questions:

1) Can real-time EMG-driven modelling achieve accuracy in joint torques close to the golden standard (Inverse dynamics)?

2) Can real-time EMG-driven modelling achieve computation time within EMD?

3) Can EMG-driven modelling extrapolate outside of its calibration data?

Locomotion is crucial for giving back independence to paretic patients but walking with an exoskeleton can be arduous due to the added weight, kinematic constraints, and resistive force due to imperfect torque controllers. Thus, computing joint torques in real-time, as well as internal body parameters such as muscle forces during walking with an exoskeleton, can be challenging. It is unknown if the added inertia, weight, and assistance provided by the wearable robot could invalidate the predicted torque from the EMG-driven model. It is important to ensure the validity of the model since once the joint torque is computed, it will be used as assistance to support different locomotion modalities while wearing the exoskeleton. Moreover, assisting locomotion with an exoskeleton is extremely challenging [29]. Current research has shown that when using optimized torque profile assistance, metabolic consumption reduction can be obtained [29], [30]. Unfortunately, the limitations are multiple such as the torque profile being only valid for the task it was optimized on and the need for a long optimization process taking up hours of walking, which may not be feasible on patients.

From this, we have deducted the following research question:

4) Can predicted joint torques from EMG-driven modelling offer reduction in EMG and joint torques levels when used to assist via an exoskeleton healthy users during diverse locomotion modalities?

Finally, the goal of this dissertation being to improve patients' mobility, experiments with the developed HMI on patients have to be done. This is extremely challenging for mainly two reasons. The first one being that to obtain the best results, the gold-standard calibration for the neuromusculoskeletal model requires diverse dynamic tasks like walking, squatting and so on. Unfortunately, this is not always feasible for all patients. So a different way of accessing patients' data for calibration has to be developed. For that, the exoskeleton was used as a dynamometer and the recorded isometric tasks were used for calibration of the neuromusculoskeletal model. Then, the validity of the assistance delivered by the HMI, which is proportional to the predicted joint torques has to be accessed. It is unknown if joint torques computed by EMG-driven model driven by pathological EMG signals will allow positive assistance and reduction in EMG amplitudes and variabilities.

From this, we have deducted the following research questions: 
5) Can the developed HMI allow paretic patients to voluntarily control a robotic device?

6) Can assistance via a robotic device based on EMG-driven modelling provide EMG amplitude and variability reductions in paretic subjects?

\subsection{Outline of this dissertation}

The dissertation follows the outline presented in the next paragraphs.

The second chapter presents the development of a real-time EMG-driven model algorithm that validates the research questions 1) and 2) presented in the previous section. The possibility to use only a subset of all calibration tasks was also tested to validate the extrapolation capability of our HMI.

The third chapter presents the combination of the Achilles ankle exoskeleton [31] (see Section 7.1.1.1.1 for a detailed description of the device) with our HMI to compute joint torques and internal body parameters such as muscle forces. This chapter also shows how muscle-tendon units were altered by the exoskeleton's assistance. This chapter answers mainly research question 3).

The fourth chapter presents the combination of our HMI with the H2 exoskeleton [32] (see Section 7.1.1.1.2 for a detailed description of the device). The possibility of calibrating our model on patients and having them receiving assistance in real-time based on their joint torques was investigated. Validation on seated tasks close to the ones done during rehabilitation therapy was realized. EMG amplitude reduction and EMG variability were used to assess if a benefit was given to the patient. This chapter answers our research questions 4) and 5).

The fifth chapter presents the combination with the WE2 [33] exoskeleton (see Section 7.1.1.1.3 for a detailed description of the device) and our HMI. The possibility of assisting during different locomotion modalities using the computed joint torques was tested. The walking tasks included two different speeds on three different inclinations in one long recording to also evaluate the transition capability between modalities. Validations of the results were done by looking at the reduction obtained at the level of EMGs and biological joint torques. This chapter answers our research questions 2), 3), 4) and 5).

The sixth chapter presents an overall discussion of the key findings and their impact on the scientific community, the limitations of this work, the directions for future research, and a short conclusion. 


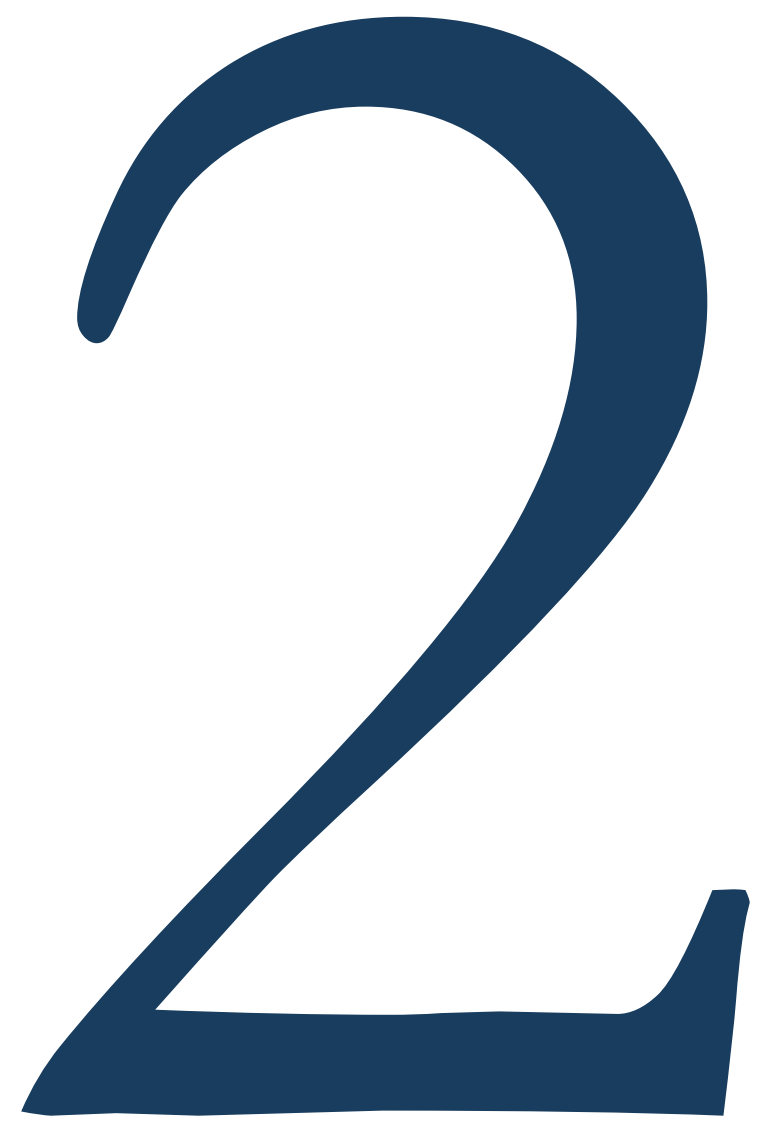




\title{
ROBUST REAL-TIME MUSCULOSKELETAL MODELLING DRIVEN BY ELECTROMYOGRAMS
}

\section{Guillaume Durandau, Dario Farina, Massimo Sartori}

\begin{abstract}
Current clinical biomechanics involves lengthy data acquisition and timeconsuming offline analyses with biomechanical models not operating in real-time for manmachine interfacing. We developed a method that enables online analysis of neuromusculoskeletal function in vivo in the intact human. We used electromyography (EMG)-driven musculoskeletal modelling to simulate all transformations from muscle excitation onset (EMGs) to mechanical moment production around multiple lower-limb degrees of freedom (DOFs). We developed a calibration algorithm that enables adjusting musculoskeletal model parameters specific to an individual's anthropometry and forcegenerating capacity. We incorporated the modelling paradigm into a computationally efficient, generic framework that can be interfaced in real-time with any movement data collection system. The framework demonstrated the ability to compute forces in 13 lowerlimb muscle-tendon units and resulting moments about three joint DOFs simultaneously in real-time. Remarkably, it was capable of extrapolating beyond calibration conditions, i.e. predicting accurate joint moments during six unseen tasks and one unseen DOF. The proposed framework can dramatically reduce evaluation latency in current clinical biomechanics and open up new avenues for establishing prompt and personalized treatments, as well as for establishing natural interfaces between patients and rehabilitation systems. The integration of EMG with numerical modelling will enable simulating realistic neuromuscular strategies in conditions including muscular/orthopaedic deficit, which could not be robustly simulated via pure modelling formulations. This will enable translation to clinical settings and development of healthcare technologies including real-time bio-feedback of internal mechanical forces and direct patient-machine interfacing.
\end{abstract}

Keywords_Electromyography; Extrapolation; Joint Moment; Musculoskeletal Modeling; Real-Time.

Publication-G. Durandau, D. Farina and M. Sartori, "Robust Real-Time Musculoskeletal Modeling Driven by Electromyograms," in IEEE Transactions on Biomedical Engineering, vol. 65, no. 3, pp. 556-564, March 2018. 


\subsection{Introduction}

Studying the neuromusculoskeletal (NMS) mechanisms underlying human movement is a fundamental challenge. This is central to characterize movement function and how it alters with pathology, thus providing a basis for devising personalized treatments. The study of human movement typically starts from the recording of experimental data including wholebody kinematics, foot-ground reaction forces (GRFs) and muscle electromyograms (EMG). Computational NMS models and simulations can be subsequently established to track experimental recordings, i.e. EMGs, GRFs, and marker trajectories [34]. This enables accessing internal body variables that are not easily measured experimentally [35], e.g. muscle force [36] or joint loadings [15].

Musculoskeletal models based on inverse dynamics are currently operated offline and available in software packages such as OpenSim [25], AnyBody [37] and Biomechanics of Bodies ${ }^{14}$. Recent studies proposed online solutions, facilitating translation to clinical scenarios [38], [39]. In these methods, the multi-muscle recruitment problem is solved by navigating the solution space and selecting one muscle activation strategy that is optimal according to a priori defined physiological criteria, i.e. the minimal sum of squared activation [40]. However, pre-defined criteria cannot encompass an individual's entire neuromuscular repertoire and its adaptations across conditions [41]. This motivated forward dynamics

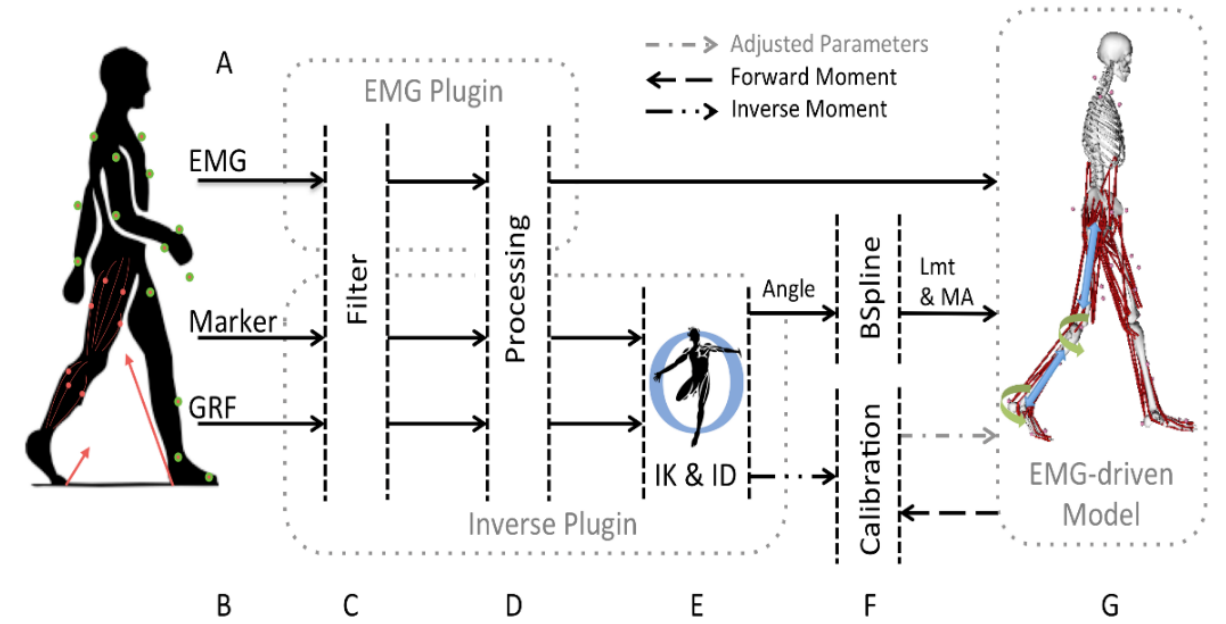

Figure 2-1: Schematics of the modeling framework. It is composed of four main parts including: movement data recording (A, B), plug-in system for data processing (C-E, with real-time inverse kinematics and inverse dynamics), musculoskeletal model calibration procedure and the real-time EMG-driven musculoskeletal modeling (F, G). The Calibration procedure and the BSpline coefficients computation are performed offline. Also see Section II.

\footnotetext{
14 http://www.prosim.co.uk/BoB/
} 
methods where EMG is combined with numerical simulation to account for realistic neuromuscular strategies without making assumptions on muscle recruitment. These are referred to as EMG-driven musculoskeletal models [14], [22], [42]-[44]. The authors and colleagues have developed and used them for estimating internal body forces [22] tightly depend on multi-muscle co-excitation, such as joint loadings [15], [45] or joint stiffness [46], [47], where inverse dynamics methods would be challenged [48], [49].

Online EMG-driven modelling has been so far proposed and tested only in restricted conditions, i.e. about one single-degree of freedom (DOF) only [23], [24], [27], [28], on isometric tasks [27], and validated on the same tasks used for model calibration. Moreover, current online formulations did not model the full force-length-velocity properties of muscles [23], [24], [27], [28]. This all would prevent robust translation of these solutions to real-world applications. Although a real-time two-DOF upper limb model was recently proposed [50], this was not driven by actual voluntary EMGs but operated via synthetic simulated signals. Moreover, it was tested for computational speed on a desktop computer and was not validated on the ability of blindly predicting internal joint forces.

We propose for the first time, an EMG-driven musculoskeletal modelling framework, that enables operating any musculoskeletal geometry model online and simulating the dynamics of multiple skeletal DOFs simultaneously. We tested the framework on the ability to predict joint moments from motor tasks and DOFs that were not used for calibration, demonstrating extrapolation capacity. We also demonstrated that the framework can operate online on low power embedded systems with computational latencies that are within the physiological electromechanical delay (EMDs). Our framework realizes processing steps that

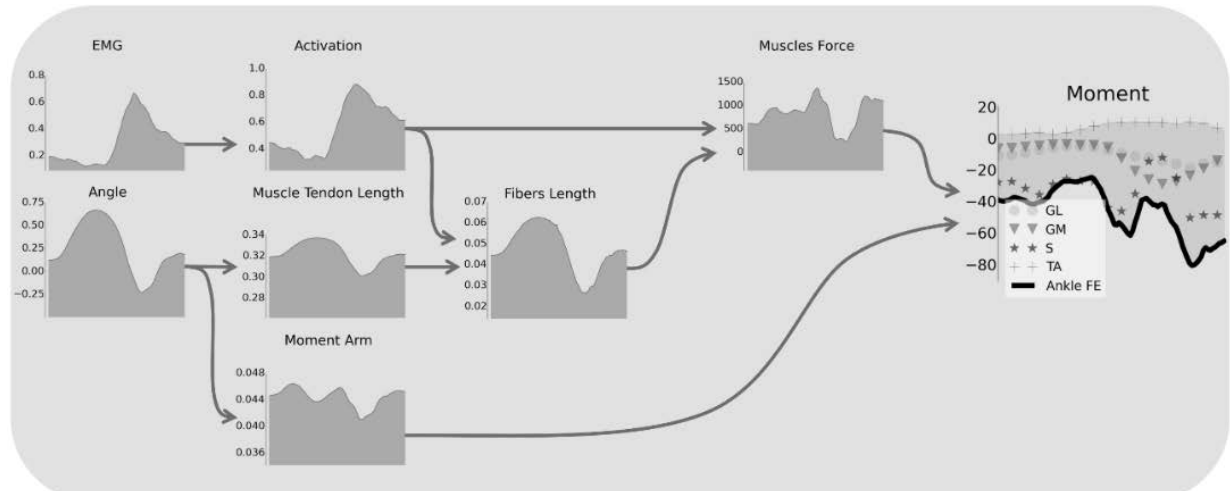

Figure 2-2: Workflow of the EMG-driven musculoskeletal modeling pipeline. From EMGexcitations and joint angles to predicted internal joint moments. The diagram depicts, representatively, Soleus muscle variables and the net joint moment contributed by the muscles spanning the ankle plantar-dorsi flexion DOF. Angles are in radians, the EMG-excitations and activation are normalized. Fiber length, LMT and MA are in meter. Muscle forces are in Newton and joint moments in Newton-meter. 
are normally performed by multiple software tools while providing real-time access to internal body variables, such as muscle activation, fiber length, contraction velocity as well as musculotendon length (Lmt), moment arm (MA), force and resulting net joint DOF moments. To enable further use in the scientific community we provide open-access to movement data and simulation at simtk.org ${ }^{15}$.

The paper is organized as follows: Section II presents the model structure and architecture. Section III presents the experiments conducted. Section IV-VI provide results, discussion and conclusion remarks.

\subsection{Real-time EMG-Driven Modelling}

We developed a real-time musculoskeletal modelling pipeline driven by measured EMGs and motion-capture data based on our previous work (Fig. 1) [14], [21], [51]. The pipeline first stage (see $I K$ \& ID in Fig. 1E) is based on a mathematical representation of the dynamics and kinematics of the human whole-body encompassing 23 DOFs. The second stage (see BSpline in Fig. 1F), uses lower extremity joint kinematics (6 DOFs) to determine the underlying muscle-tendon kinematics, i.e. Lmt and MA. The third stage (see EMG-driven model in Fig. 1G), uses EMGs in conjunction with muscle-tendon kinematics to compute musculotendon force and resulting joint moments in the knee and ankle joints (Fig. 2).

The real-time framework was developed in ANSI C++ (Fig. 1). It comprises two plug-in modules for direct connection with external recording devices (Fig. 1A-B) and with the OpenSim application programming interface (API, Fig. 1E-F). Moreover, it comprises a modelling component for the computation of musculotendon kinematics based on our previously developed Multidimensional Cubic BSpline (MCBS) method (Fig. 1F) [21] as well as a component for the simulation of musculotendon dynamics based on the previously developed Calibrated EMG-informed Neuromusculoskeletal Modeling (CEINMS) method [14], [51] (Fig. 1G).

\subsubsection{Software Plug-In}

The first plug-in module enables TCP/IP direct connection to external EMG amplifiers (Fig. 1A). It records raw EMGs and extracts amplitude-normalized linear envelopes. The processing steps include high-pass filtering, full-wave rectification, and low-pass filtering. For each subject and muscle, the resulting EMG linear envelopes were amplitude-normalized with respect to the peak-processed values obtained from the entire set of recorded trials including both isometric maximal voluntary contractions (MVCs) and dynamic trials. This assured EMG linear envelopes always varied between 0 and 1 , an important requirement for

\footnotetext{
15 https://simtk.org/projects/rems
} 

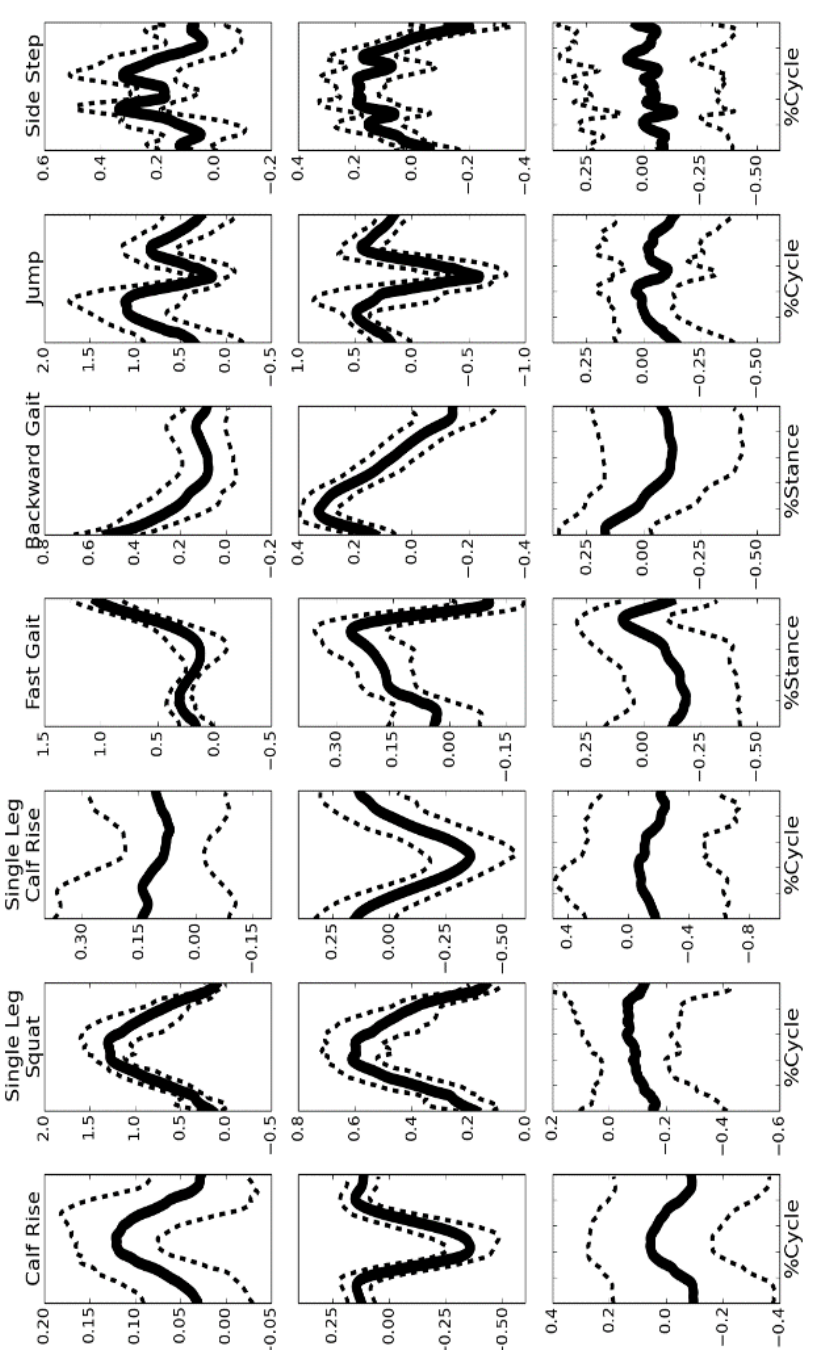

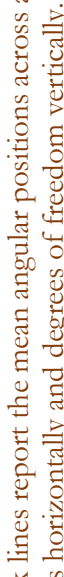

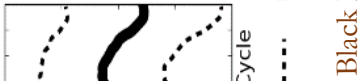

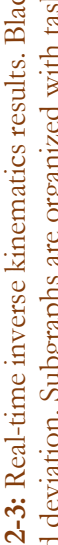
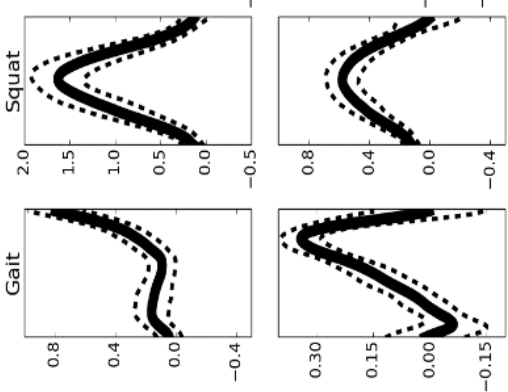

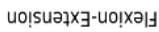

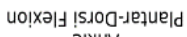

sueppey
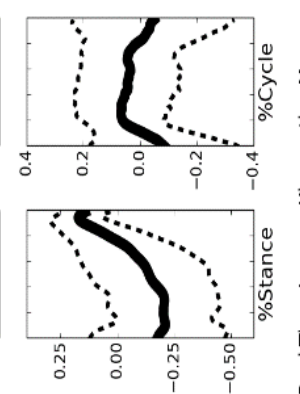

uo!xəəy deeq도 ə|주

告 
musculotendon unit (MTU) force-production modelling. Filtered and amplitude-normalized EMGs will be referred to as muscle excitations. The second plug-in module enables TCP/IP direct connection to external motion capture (MOCAP) systems (Fig. 1B). It records and processes three-dimensional marker trajectories and GRFs to derive joint angle and joint moment estimates via real-time inverse kinematics (IK) and inverse dynamics (ID) performed using the OpenSim API. The module low pass filters the three-dimensional marker trajectories and rotates them from the MOCAP system reference frame into the OpenSim reference frame (Fig. 1D). The OpenSim model used for the IK and ID procedure is taken from [52] and comprises 23 DOFs.

We extended the OpenSim single-thread IK algorithm into a multi-thread algorithm that produced real-time estimates (i.e., at $100 \mathrm{~Hz}$ ) of three-dimensional joint angles from filtered, rotated marker trajectories (Fig. 1E). In this, we established a direct TCP/IP connection to the MOCAP system to record markers trajectories and stream them to the OpenSim API framework (Fig 1B-E). The IK problem in OpenSim is solved via static optimization. For each time frame, three-dimensional joint angles are computed to minimize the root mean squared error (RMSE) between a set of virtual markers attached to the OpenSim musculoskeletal model anatomical landmarks and the corresponding set of experimental markers placed on the same anatomical landmarks of each subject [25]. To obtain real-time IK capability, we ran simultaneously multiple optimizations on different threads within a multi-stage pipeline. When a single frame of experimental marker trajectory is received, it is assigned to one thread, which performs one IK optimization. When a new experimental marker trajectory is received and the previous thread has not yet completed by the IK optimization, a new thread is established to perform concurrent optimization. The initial parameters used for the up-coming optimization stage are the latest computed DOF angles available. The plug-in also records experimental GRFs, low pass filters them and computes the resulting foot-ground center of pressure (COP, Fig. 1C). Filtered GRFs and COPs are rotated from the force plate reference frame into the OpenSim reference frame (Fig. 1D). The plug-in employs a Kalman filter [53] to process IK-generated joint angles and computes dynamically consistent estimates of joint angular velocity and acceleration (Fig. 1D). The Kalman filter parameters are derived as described previously [53]. Filtered and rotated GRFs, COPs, as well as Kalman, filtered joint angle, velocity and acceleration are streamed to the OpenSim API for the ID calculation and subsequent computation of the resulting joint moments (Fig. 1E). We refer to these to as the "experimental moment". 

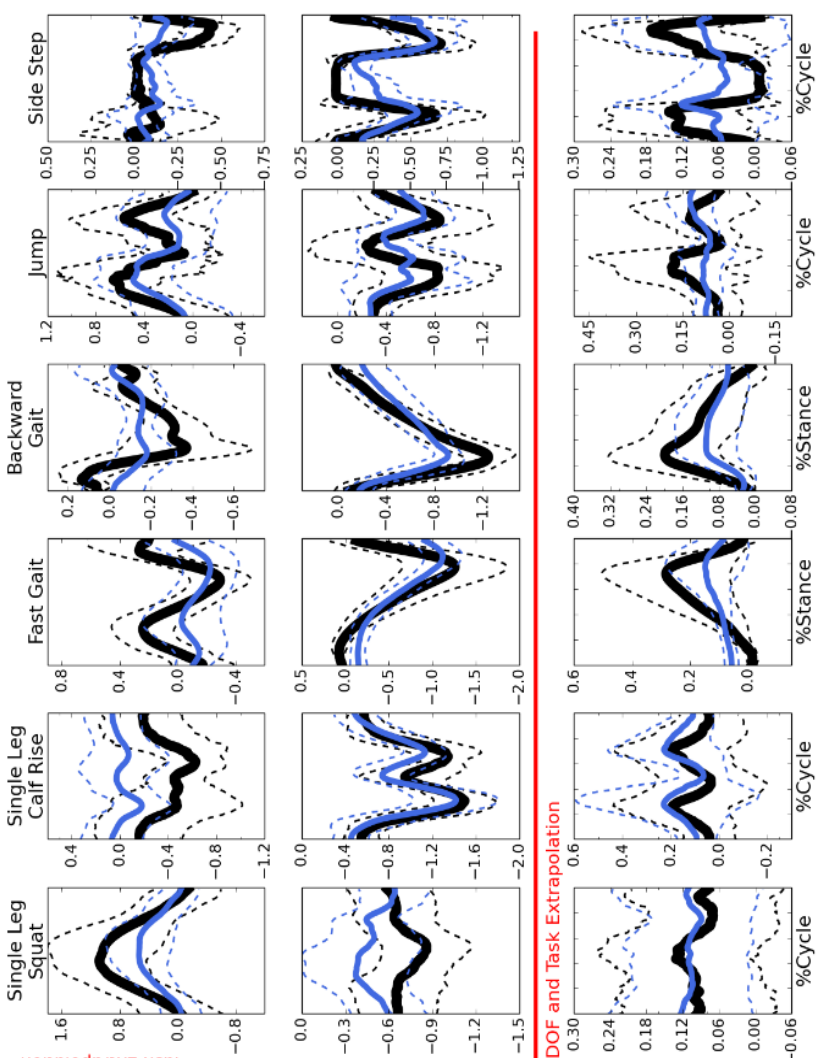

롱.

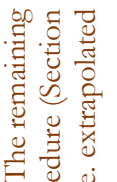

F

式出

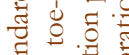

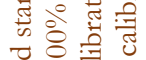

专苑苍

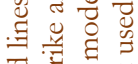

:풍 范

局过

घ. ज्ञ

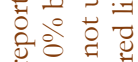

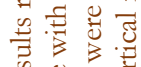

过

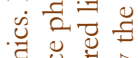

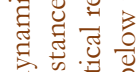

of

峁苛㝴

芯矛过

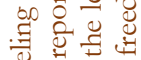
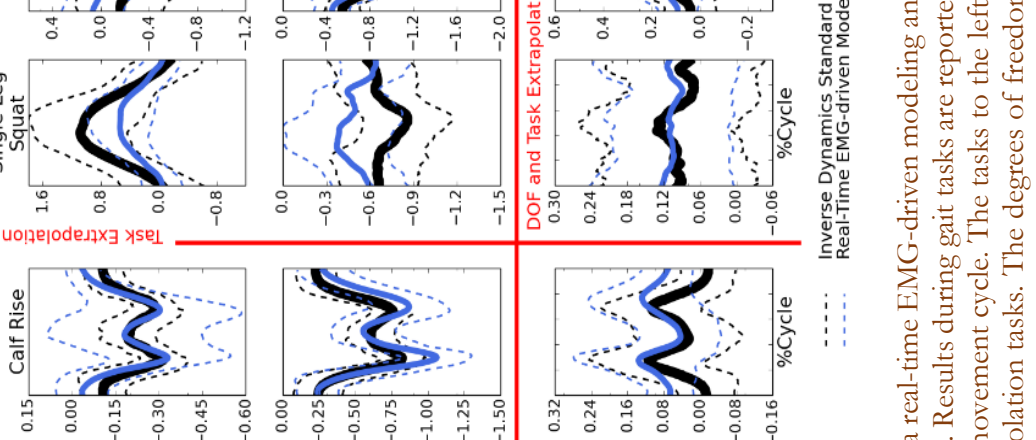

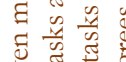

吾.

b

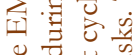
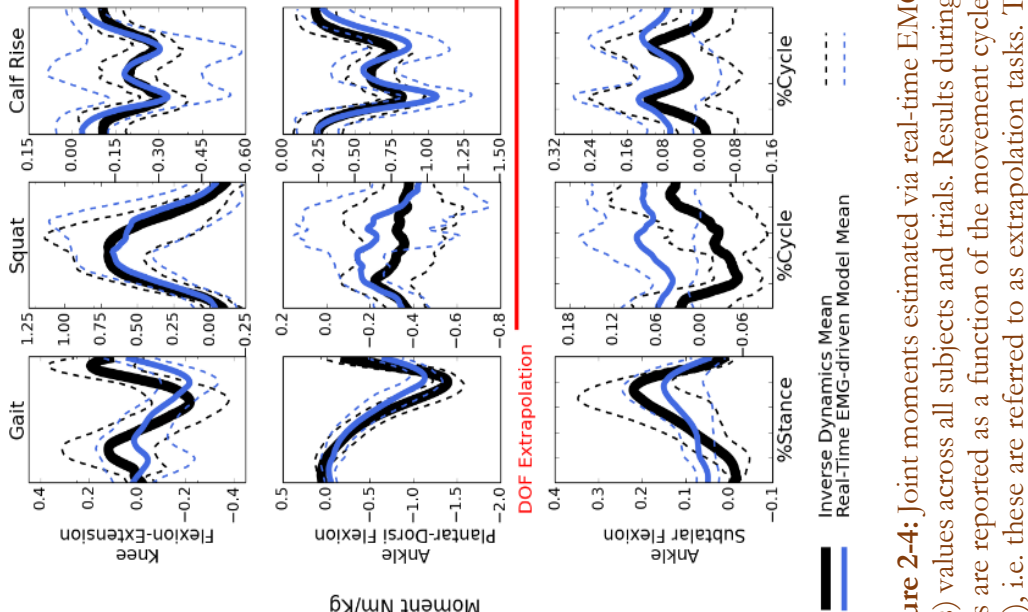

6x/mn zuәmow

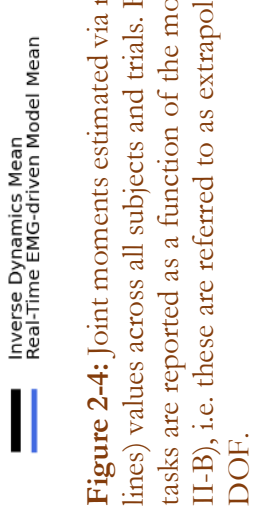




\subsubsection{EMG-driven modeling}

The alternative pathway to joint moments is via EMG-driven musculoskeletal modelling (Fig. $1 G)$. In this scheme, the same musculoskeletal geometry model used for the IK and ID calculations is employed (Section III). We computed EMG-dependent forces for 13 MTUs spanning the knee and ankle joints. These included: semimembranosus, semitendinosus, biceps femoris long and short head, tensor fasciae latae, rectus femoris, vastus medialis, vastus intermedius, vastus lateralis, gastrocnemius medialis, gastrocnemius lateralis, soleus and tibialis anterior. We used a subset of the IK-generated whole-body angle estimates. These are six lower extremity DOFs defining the kinematics of the 13 selected MTUs, including subtalar flexion, ankle flexion-extension, knee flexion-extension, hip abduction, hip flexion-extension, and hip internal-external rotation [52]. IK-generated joint angles about the six selected DOFs are used to determine the underlying MTU kinematics, i.e. Lmt and MA (Fig. 1F). To achieve real-time performance we integrated into our framework the MCBS method we previously developed [21] (Fig. 1F). This synthesizes the complex MTU paths defined in large-scale OpenSim musculoskeletal geometry models into a set of MTU-specific multidimensional cubic Bsplines. This enables accurate computation of kinematic-dependent length and moment arms for all MTUs at the fastest computational speed to date, allowing the use of embedded systems with limited power.

EMG-excitation, Lmt and MA estimates are then used to compute EMG-dependent MTU force and joint moment estimates (Figs $1 G$ and 2). EMG-excitations are processed via a non-linear transfer function to determine the muscle fiber twitch dynamics in response to EMG-derived muscle excitation, as previously proposed [54]. Tendons were modelled as fiber series elements of constant tendon slack length. Resulting musculotendon forces were

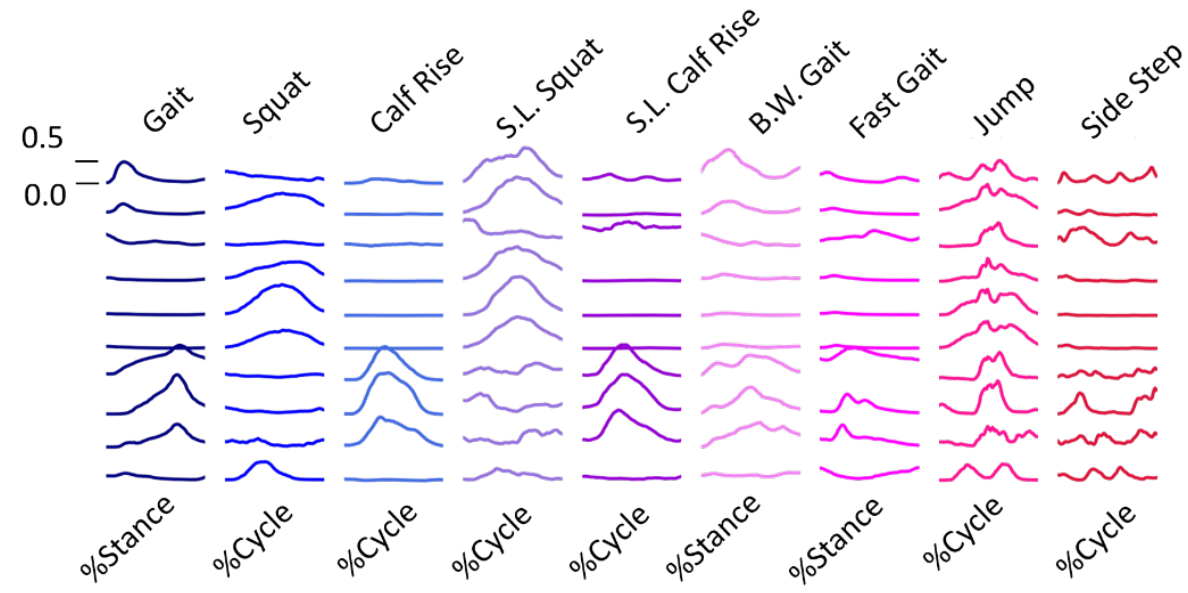

Figure 2-5: Filtered and normalized EMGs across all tasks for muscles including (from top to bottom): semimembranosus, biceps femoris, tensor fasciae latae, rectus femoris, vastus medialis, vastus laterals, gastrocnemius lateralis, gastrocnemius medialis, soleus and tibia anterior. 
transferred to the joint via moment arms with no modelled ligament contribution. This enabled substantial computation speed with little to no loss of accuracy with respect to elastic tendon elements in the estimation of joint moments, as we previously proved [55].

We developed a calibration procedure for deriving MTU parameters that determine subject-specific MTU-force generating capacity and that vary nonlinearly with subject anthropometry (Fig. 1F). These included MTU-specific optimal fiber length and tendon slack length, grouped maximal muscle forces, and a global excitation-to-activation shape factor [35]. In the first stage, the calibration procedure computes BSpline coefficients necessary for the estimation of Lmt and MA. The OpenSim API is used to derive Lmt nominal values for all MTUs spanning the ankle subtalar-flexion, ankle flexion-extension and knee flexionextension DOFs. Using these data, the piecewise polynomial coefficients are computed for every order of the BSpline. The order of the BSpline depends on the number of DOFs crossed by an MTU. The second stage determines subject-specific values of optimal fiber length and tendon slack length specifically for each MTU, as previously described in [56]. An optimization procedure determines tendon slack length and optimal fiber length values so that normalized muscle fiber length and tendon strain between the scaled and unscaled musculoskeletal geometry models are preserved across DOF functional operating ranges [56]. The third stage uses a constraint optimization to vary between pre-defined boundaries the EMG-to-activation shape factor parameter (i.e. between -3 and 0 ), the MTU maximal isometric force (i.e. scaled by factors between 0.5 and 1.5) and further refine the previous estimates of optimal fiber length (i.e. within $\pm 2.5 \%$ of its initial value) and tendon slack

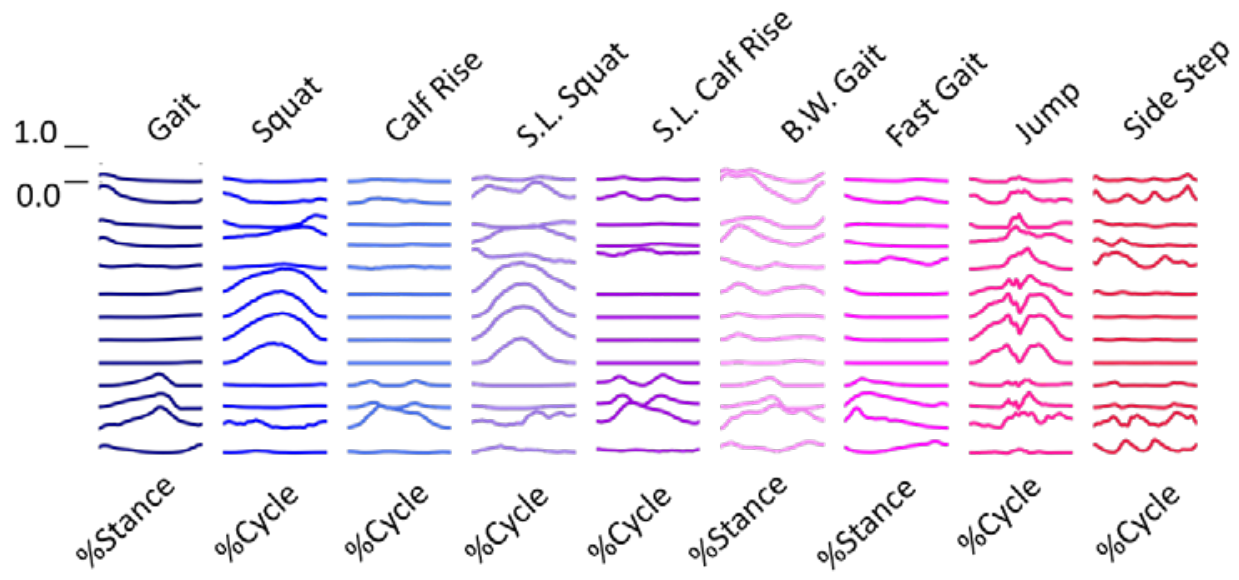

Figure 2-6: Normalized musculotendon unit (MTU) force computed via the EMG-driven model across all tasks for MTUs including (from top to bottom) the semimembranosus, semitendinosus, biceps femoris long head, biceps femoris short head (BFS), tensor fasciae latae, rectus femoris, vastus medial, vastus intermedius, vastus lateralis, gastrocnemius medialis, gastrocnemius lateralis, soleus, tibia anterior. 
Table 2-1: Participants' Anthropometric Properties and Locomotion Speed.

\begin{tabular}{ccccccc}
\hline \multirow{2}{*}{ Participant } & \multirow{2}{*}{$\begin{array}{c}\text { Age } \\
\text { (years) }\end{array}$} & Height & Weight & \multicolumn{3}{c}{ Gait Speed (m/s) } \\
\cline { 5 - 7 } & 26 & 1.77 & 73 & 0.66 & 0.74 & 0.59 \\
\cline { 5 - 7 } & 26 & 1.82 & 70 & 0.68 & 0.93 & 0.43 \\
2 & 31 & & Free & Fast & Backward \\
3 & 34 & 1.82 & 67 & 0.65 & 0.70 & 0.31 \\
4 & 29 & 1.71 & 73 & 0.58 & 0.84 & 0.49 \\
5 & 28 & 1.86 & 85 & 0.66 & 0.96 & 0.63 \\
\hline
\end{tabular}

length (i.e. within $\pm 5 \%$ of its initial value). Parameters are varied using a simulated annealing procedure [57] until the discrepancy between experimental and predicted joint moments is minimized over a range of calibration trials (Section III). We developed a graphical user interface $(\mathrm{GUI})$ to enable real-time visual feedback of modeling steps including IK and ID calculations, EMG-muscle excitation processing as well as EMG-driven model-based estimation of MTU and joint variables. The video available in the supplementary material shows the real-time modeling framework being used on one individual subject.

\subsection{Experimental Procedures}

The University Medical Center Göttingen Ethical Committee approved all experimental procedures. Five healthy men (see Table I) volunteered for this investigation after providing signed informed consent. Data were recorded and processed in real-time using the modelling framework described in Section II, depicted in Fig. 1, and displayed in the supplementary video.

EMGs were recorded using a 256-channel EMG amplifier (OTBioelettronica, Italy) at $2048 \mathrm{~Hz}$. The high-pass filter was a second-order Butterworth filter with $30 \mathrm{~Hz}$ cut-off. The low-pass filter was a second-order Butterworth with a $4 \mathrm{~Hz}$ cut-off. We recorded EMG signals from 10 muscle groups including: rectus femoris, lateral and medial hamstrings, vastus medialis and lateralis, tensor fasciae latae, gastrocnemious medialis and lateralis, soleus and tibialis anterior. Muscle group EMGs were allocated to individual MTUs defined in the modelling framework (Section II.B). In this allocation, two MTUs that shared the same innervation and contributed to the same mechanical action were assumed to have the same EMG pattern. According to this convention, the lateral hamstring EMGs drove both the biceps femoris short head and long head MTUs. The medial hamstring EMGs drove both the semimembranosus and the semitendinosus MTUs. The vastus intermedius EMG activity was derived as the mean between the vastus lateralis and vastus medialis EMGs [14]. All remaining MTUs had dedicated EMG channels. A set of 29 retroreflective markers was placed on the trunk and lower extremity, as previously described [14]. Three-dimensional marker trajectories were recorded using a seven-camera motion capture system (Qualisys, 
Göteborg, Sweden) at $128 \mathrm{~Hz}$. Foot-ground reaction forces (GRFs) were recorded using two in-ground force plates (Bertec, Columbus, USA) at 2048Hz. The low-pass filter used for both marker and GRF data was a second-order Butterworth filter with $10 \mathrm{~Hz}$ cut-off and a time group delay of $0.1-25 \mathrm{~ms}$ with average delays in the order of $20 \mathrm{~ms}$.

The subjects performed a static standing trial. The recorded marker trajectories were used to scale an OpenSim generic musculoskeletal model to match each individual subject's anthropometry. MVC trials consisting of isometric contractions were performed for each muscle group for EMG normalization. The subjects performed three model calibration trials including one static standing trial, one single repetition of forward gait at a self-selected speed, and one single repetition of knee squat followed by calf rise. The model calibration procedure (Section II) was performed to minimize the discrepancy between predicted and experimental moments about the knee flexion-extension and ankle plantar-dorsiflexion DOFs. The subtalar-flexion DOF was not included in the calibration procedure. Validation trials included five additional repetitions of the calibration tasks (excluding the static standing task) as well as five repetitions of novel motor tasks including: backward gait at a self-selected speed, fast forward gait, knee squat, single-leg knee squat, calf rise, single-leg calf rise, knee squat followed by a vertical jump, and sidestepping. Motor tasks were chosen to underlie a variety of different neuromuscular strategies and produce a range of dynamic joint moments across knee and ankle joint DOFs.

The whole real-time modelling framework (i.e. EMG-processing, IK, ID, and EMGdriven modelling, Fig. 1) was operated on a laptop with dual-core processing unit $(2.60 \mathrm{GHz})$ and $16 \mathrm{~GB}$ of RAM memory. Tests were also repeated using an embedded system (Raspberry Pi 2, Raspberry Pi Foundation, UK), which is a single-board computer with a four-core processing unit $(900 \mathrm{MHz})$ and $1 \mathrm{~GB}$ of RAM memory. In this, joint angles and EMGs were read from file, i.e. we did not employ real-time EMG processing and IK computation. Three tests were performed for validating the framework capabilities.

\subsection{Results}

The first test verified the framework ability of computing joint angular positions in real-time via IK. Angles estimates about 23 articular joint DOFs were produced at an average rate of $168 \pm 141 \mathrm{~Hz}$. Fig. 3 reports values derived about the knee flexion-extension, ankle plantardorsiflexion, and ankle subtalar flexion across all motor tasks. These are the DOFs employed in the subsequent EMG-driven modelling pipeline. IK-generated angles reflect literature values across forward gait [58], backward gait [36], and squat tasks [59]. Table I summarizes locomotion speeds performed by all subjects as well as each individual's anthropometry properties. Table I also show how self-selected locomotion speeds largely varied across

participants generating a variety of different motor conditions to be predicted by the framework. 


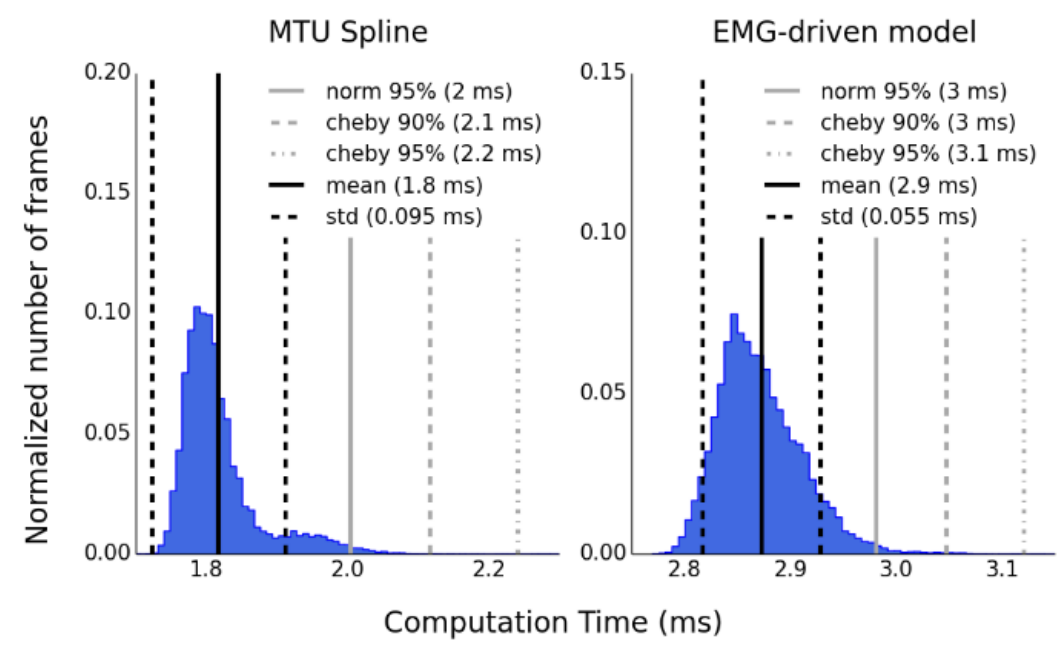

Figure 2-7: Computation time on the Raspberry Pi 2. The left-hand histogram depicts the computation time of MTU spline component. The right-hand histogram depicts the computation time of the EMG-driven model component.

The second test (Fig. 4) verified the real-time framework ability to estimate joint moments in real-time using the EMG-driven modelling pipeline using experimental EMG-excitations (Fig. 2) and IK-angles (Fig. 3). Results showed estimated joint moments being in agreement with ID generated joint moments (reference) derived using experimental GRFs and IK angles. Fig. 4 shows the model ability to predict moments during novel repetitions of the calibration trials including gait at a self-selected speed, knee squat with subsequent calf rise. Moreover, Fig. 4 also shows the model ability to extrapolate beyond calibration conditions. That is, to completely unseen motor tasks (i.e. extrapolation capacity: backward gait, sidestep, single-leg squat with calf rise, fast gait and vertical jump), and about one unseen DOF (i.e. ankle subtalar flexion). The largest Pearson coefficients $r=0.9 \pm 0.07$ was observed at the ankle plantar-dorsiflexion DOF during gait at a self-selected speed. The smallest root mean square error (RMSE) was observed at the subtalar flexion DOF $(0.01 \pm 0.01 \mathrm{Nm} / \mathrm{kg})$ during the single-leg squat task. Pearson coefficients were always greater than $r=0.43 \pm 0.36$ with least favorable values observed at the knee flexion-extension DOF during gait at a selfselected speed. The RMSE was always smaller than $0.37 \pm 0.12 \mathrm{Nm} / \mathrm{kg}$ with the least favorable values observed at the knee flexion-extension DOF during the single-leg calf rise task. The EMG-driven model prediction accuracy during the unseen motor tasks was comparable to that observed during novel trials of the same type used for calibration. The RMSE and $r$ variation from calibration to extrapolation trials was $0.02 \mathrm{Nm} / \mathrm{kg}$ and 0.07 respectively at the knee flexion-extension, $0.003 \mathrm{Nm} / \mathrm{kg}$ and 0.06 at the ankle plantar-dorsiflexion, and $0.25 \mathrm{Nm} / \mathrm{kg}$ and 0.12 at the ankle subtalar flexion. The task that displayed the largest prediction accuracy variation between calibration and extrapolation tasks was the single-leg knee squat with calf rise. The joint moments predicted both using EMG-driven modelling 
and ID well reflected normative values found in the literature for tasks including gait [60], backward gait [61] and squat [62].

Fig. 5 shows the EMG-excitations used for joint moment prediction across all tasks and muscles and reported for one subject. Excitations were found to assume values comparable for the forward gait[63], backward gait [64] and squat [62], for which literature data are available. During the knee squat, excitations from the quadriceps group assumed substantially high values in the knee extension part of the task. Similarly, calf muscle excitations assumed larger values during the calf raising part of the tasks. The jump task had comparable excitation patterns to the squat task particularly at the beginning and the end of the task.

Fig. 6 shows the normalized force predicted for all MTUs across all motor tasks and reported for one subject. Results showed values matching literature data for gait [65] and squat tasks [62] for which values for comparison are available. Importantly, Fig. 5 and 6 highlight the non-proportionality existing between EMG-excitations and resulting forces, where modulations in EMG-excitations does not always correspond to a linear modulation at the force level. This reflects the non-linear EMG-to-activation transfer function (Section II) and the Hill-type viscoelasticity via force-length-velocity relationship.

The third test (Figs 7 and 8) quantified the framework real-time computation performance when operated both on a laboratory desktop computer and on an embedded system. We used metrics including: the mean computation time and standard deviation measured across all simulation frames from all subjects and tasks, the maximal expected computation time within a 95\% confidence interval assuming computation time frames with a normal Gaussian
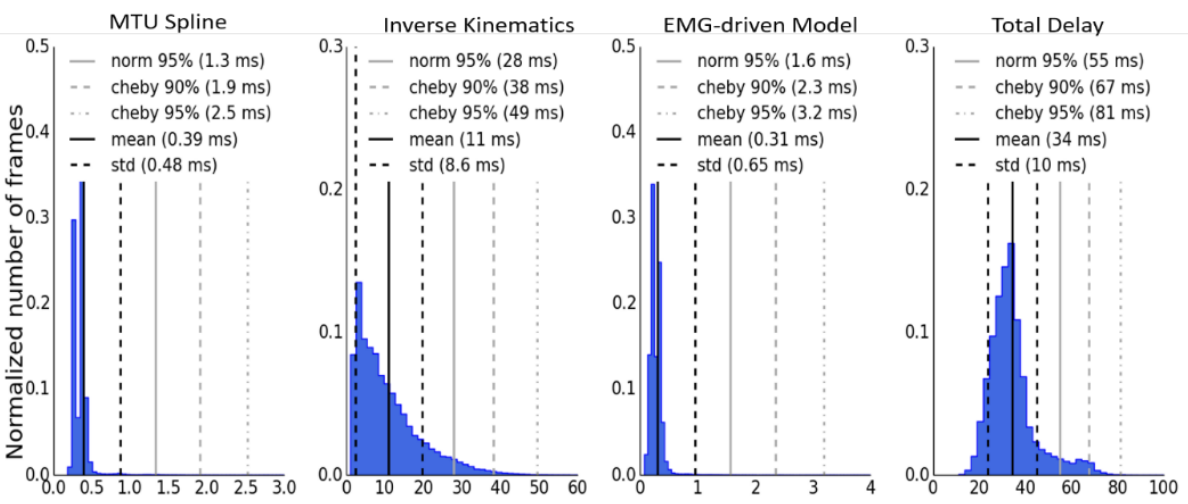

Computation Time (ms)

Figure 2-8: Computation time on a desktop computer. The histograms (starting from left) respectively depict computation times for the MTU spline, inverse kinematics, and EMG-driven model component as well as the total delay between EMG sampling time and multi-DOF moment computation. The inverse dynamics computation time is not reported as this is constant and does not add substantial latency to the workflow. 
distribution, and the maximal expected computation time with a $90 \%$ confidence interval with no assumption on the computation time frame distribution, i.e. using the Chebyshev's Theorem. Fig. 7 shows the computation time of the different components of the real-time EMG-driven pipeline (Fig. 1) on a desktop computer.

The MTU kinematics component (Fig. 1F) executed with a mean computation time of $0.4 \pm 0.47 \mathrm{~ms}$ with $95 \%$ of the samples being computed within $1.5 \mathrm{~ms}$. The inverse kinematics component (Fig. 1E) executed with a computational time of $10.1 \pm 8.5 \mathrm{~ms}$ with $95 \%$ of the samples being computed within $28 \mathrm{~ms}$. The EMG-driven model (Fig. 1G) executed in $0.301 \pm 0.65 \mathrm{~ms}$ with $95 \%$ of the samples being produced within $1.6 \mathrm{~ms}$. Fig. 7 also shows the total delay from the EMG recording time to the multi-DOF moment computation, with the mean delay being $35 \pm 11 \mathrm{~ms}$ and with $95 \%$ of the samples being produced within $55 \mathrm{~ms}$.

Fig. 8 shows the computational time of the EMG-driven model and the MTU spline on the Raspberry Pi 2 embedded system. The MTU kinematics component (Fig. 1F) operated in $4.3 \pm 0.2 \mathrm{~ms}$ with $95 \%$ of the samples being produced within $4.7 \mathrm{~ms}$. The EMG-driven model (Fig. 1G) operated in $2.7 \pm 0.48 \mathrm{~ms}$ with $95 \%$ of the samples being produced within 3.6ms. The video in the supplementary material displays the framework data recording, processing and musculoskeletal simulation capacity in real-time.

\subsection{Discussion}

We developed and validated a real-time framework for modelling and simulating the dynamics of the human NMS system using EMG-driven modelling. The real-time framework enables recording and processing movement data (marker trajectories, GRF, EMGs) and determining reference three-dimensional joint angles and moments via real-time IK and ID. Moreover, it enables simulating how EMG-controlled muscle contractions transfer mechanical force to skeletal structures instantly during an individual's movement. In this, EMGs enable simulating realistic subject-specific neuromuscular strategies across different individuals in conditions also including muscular/orthopaedic deficit, which could not be robustly simulated via pure modelling formulations [17]. In this study, we calibrated and tested the EMG-driven modelling pipeline using a lower extremity musculoskeletal geometry model with six DOFs (Section II). However, the proposed framework enables realtime simulation of any musculoskeletal geometry model generated using the OpenSim modelling software package. ${ }^{16}$

The proposed framework enabled for the first time, robust estimation of musclecontributed joint moments about multiple DOFs simultaneously, during unseen dynamic motor tasks and DOF as well as using low power portable embedded systems. The joint moment estimation ability over the unseen motor tasks and DOFs was comparable to that

16 http://simtk-confluence.stanford.edu:8080/display/OpenSim/
Musculoskeletal+Models 
observed during novel repetition of the calibration tasks and DOFs (Section II). These results support the possibility of translating the proposed EMG-driven musculoskeletal modelling technique to real-world applications, i.e. computer-aided motor diagnosis and rehabilitation, human-machine interfacing, model-based control of assistive devices.

Results from the first test (Fig. 3) revealed large variability about the subtalar angular position highlighting this DOF predominant function of leg stabilization. This appears to be an important element, especially when comparing single leg-squat with respect to double legsquat tasks (Fig. 3). These tasks display substantially different ankle moment trends (i.e. see ID moments in Fig. 4). This is explained by single leg-squat tasks requiring larger knee joint moment production (see knee extensors function, Figs 5-6) and greater ankle stabilization function (see ankle antagonist muscle co-activation, Fig. 5). In this, estimation accuracy of fine ankle moment modulation was limited in our modelling framework by the fact that not all ankle muscles were recorded. However, despite current limits, the proposed modelling formulation was able to provide joint moment estimates in close agreement to reference data (Fig. 4). The results also revealed that the EMG-driven model better predicted the ankle plantar-dorsiflexion moments than the knee flexion-extension moments. This is due to the larger number of muscle prime movers in the knee than in the ankle. It is important to stress that the central requirement in our system was to achieve real-time performance, for which we needed to address computational challenges across all modelling and processing stages (i.e. Figs 1-2). These included on-line data acquisition and filtering introducing data losses and phase shifts respectively. In this context, we employed standard TCP/IP data acquisition protocols not specifically designed for hard real-time performances. This all limited the prediction accuracy of our system when compared to previous offline studies [14], [22], [43]. Future work will develop ad-hoc data acquisition and processing hardware and software systems, which will better enable handling real-time constraints.

Future work will investigate the real-time modelling formulation with a larger set of recorded EMG channels spanning the knee and quantify the associated model prediction sensitivity. Also, future work will explore whether high-density EMG can enable better estimates of muscle activity especially important during tasks underlying fine-control of muscle excitation and small modulations of force.

The ability to predict muscle forces while distributing their force output along all spanned DOFs simultaneously enables addressing the indeterminacy of the muscle force distribution problem, which has been achieved here for the first time in real-time. We previously showed that EMG-driven models calibrated with respect to different single-DOFs generated different MTU force solutions for the same input data and MTU set [14]. On the other hand, our proposed real-time multi-DOF modelling formulation provides a unique MTU force solution that satisfies all DOFs simultaneously and is, therefore, more generalizable across novel conditions. This was reflected by the model ability to extrapolate both task-wise and DOF-wise (Fig. 4). 
The ability to operate the modelling pipeline in real-time is important in the context of human-machine interfacing for wearable assistive devices as it would enable predicting internal body forces and the intended movement before they actually manifest in the human body. This would enable supporting individuals with reduced motor abilities but with detectable electrophysiological activity. Conversely, systems that operate on the basis of the detection of externally measurable forces (i.e., limb orthosis interaction force) would not provide support until the user has produced detectable interaction force.

Results also revealed that the elements contributing to the total computational time included: (1) the motion capture system and IK algorithm, which relies on static optimization and therefore required substantial computational power and (2) the EMG recording system we used, which sends 30ms-packets of data over TCP/IP. The EMG-driven modelling pipeline used only a small portion of the total computation time, as depicted in Figs 7-8. Future work will use fully integrated EMG and position sensors, to decrease the computational latency and assure enhanced real-time capability using wearable solutions.

The computational performance obtained using the embedded system (Fig. 8) revealed that $90 \%$ of predicted frames were computed with latencies comparable to those derived using a laptop system. Peak latencies were observed to be one order of magnitude larger than using a laptop computer system, however only in a small percentage of the cases.

Future work will investigate the possibility of predicting multiple mechanical variables including those tightly dependent on muscle co-excitation such as joint stiffness [46]. This will underlie the employment of series elastic tendon elements as previously described [46] and ligaments, thus enabling the accurate estimation of a greater range of mechanical variables. Future work will also focus on the use of co-excitation primitives [44] for relaxing sensory-constraints, i.e. the need for recording from large sets of muscles. Applications will also include the interfacing of our proposed framework with wearable assistive technologies for restoring (robotic exoskeletons) or replacing (artificial limbs) [17] lost motor capacity. In this context, previously proposed offline NMS modelling formulations demonstrated to successfully capture patient-specific musculoskeletal function in conditions including cerebral palsy [66][67], stroke [43] or quadriceps weakness [17]. In this, important muscle functional abnormalities could be modelled by optimizing the passive muscle stiffness parameters (i.e. muscle contractures) or using velocity-dependent feedback controllers (i.e. spasticity) [66].

\subsection{Conclusion}

We proposed and extensively validated an online EMG-driven musculoskeletal modelling framework that simulates the dynamics of multiple muscular and skeletal DOFs concurrently. This will enable (1) filling the gap between data collection and advanced analysis, (2) out-of-the-lab analysis for understanding human movement beyond constrained laboratory conditions, and (3) translation to the clinics and to assistive technologies. 


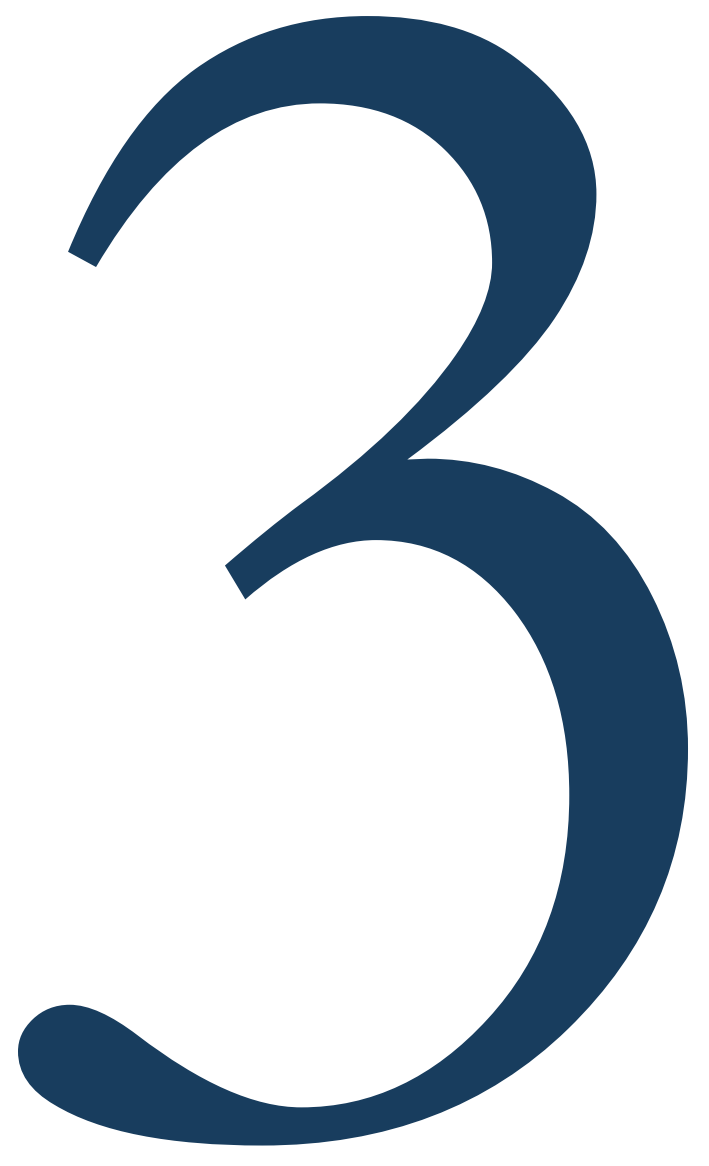




\section{TOWARD MUSCLE-DRIVEN CONTROL OF WEARABLE ROBOTS: A REAL-TIME FRAMEWORK FOR THE ESTIMATION OF NEUROMUSCULAR STATES DURING HUMAN - EXOSKELETON LOCOMOTION TASKS}

\section{Guillaume Durandau, Wolfgang Rampeltshammer, Herman van der Kooij, Massimo Sartori}

Abstract-The ability to efficiently assist human movement via wearable robotic exoskeletons requires a deep understanding of human-exoskeleton physical interaction. That is, how the exoskeleton affects human movement and how the human body reacts to robotic assistance. In this context, it is central to gain access to human neuromuscular states, i.e. neural activation to muscle, muscle fibers short-stretch cycle, tendon strain, musculotendon viscoelasticity. This would enable the personalized design of assistive devices and humanexoskeleton interfaces with respect to a specific subject's anatomy and force-generating capacity. Here we present a real-time electromyography-driven framework interfaced to a robotic bi-lateral ankle exoskeleton. This framework provides real-time information about joint and underlying muscle mechanics. We provide a quantitative evaluation of the real-time framework across a repertoire of human-exoskeleton locomotion tasks. We also show how this enables understanding of how robotic exoskeletons in parallel to human limbs contribute to alter normative musculoskeletal mechanics. This will open new avenues for the creation of symbiotic exoskeleton technologies that operate as an extension of the own body.

Keywords_Exoskeletons; Muscles; Electromyography; Real-time systems; Computational modelling.

Publication-G. Durandau, W. Rampeltshammer, H. van der Kooij and M. Sartori, "Toward Muscle-Driven Control of Wearable Robots: A Real-Time Framework for the Estimation of Neuromuscular States During Human-Exoskeleton Locomotion Tasks," 
Section: Toward Muscle-Driven Control of Wearable Robots: a Real-Time Framework for the Estimation of Neuromuscular States During HumanExoskeleton Locomotion Tasks

2018 7th IEEE International Conference on Biomedical Robotics and Biomechatronics (Biorob), Enschede, 2018, pp. 683-688. 


\subsection{Introduction}

Until very recently robotic lower-limb exoskeletons could not assist human locomotion to provide a metabolic energy cost advantage. Achievements in this direction used first a tethered actuation [68] and later untethered [69] as well as unpowered devices [70]. These techniques obtained metabolic cost reduction by prescribing pre-defined joint moment patterns to the user as a function of the gait cycle. A generalization of this was subsequently proposed via human-in-the loop optimization controllers, where the joint moments to the human are optimized online [30]. However, these methods require a large amount of metabolic data before they can adapt to variations in human gait with consequent large reaction times, i.e. $>20$ minutes [30]. As a result, current methods enable supporting constrained, pre-defined gaits with limited capability of equally well assisting different motor tasks as well as continuous transitions across tasks promptly, i.e. from locomotion to stair climbing, to stair descending.

The availability of human-machine interfaces (HMIs) that can capture human movement intention and provide prompt support across multiple movements, as well as the transition between them, is crucial for enabling wearable robotic exoskeletons to impact broad socio-economic domains, ranging from neuro-rehabilitation to industrial worker's aids, from human physical training to augmentation. A possible way to achieve this is to create HMIs that open a window into human neuromuscular states, i.e. neural activation to muscle, muscle fibers short-stretch cycle, tendon strain, musculotendon visco-elasticity. This is important as human movement directly emerges from the interplay between the nervous and musculoskeletal system [17], [71]. In this context, the ability to determine how an individual's neuro-muscular states dictate movement is central for addressing one of the major challenges in the field of wearable robotics: personalizing the design of the assistive device an HMI to a specific patient's anatomy, force-generating capacity and way of moving.

HMIs have been proposed that enable supporting different tasks via manual user interfaces based on buttons panels [72], finite sate machines coupled with wearable sensor data (i.e. from inertial measurement unit (IMU) and foot-ground contact sensors [73]), or bio-electrical signals such as electroencephalography (EEG) [74]. Impedance controller [75], [76] or sensitivity amplification control [76] offer some levels of voluntary and continuous control but no reduction in metabolic consumption. These controllers require mechanical input from the user such as interaction moments, which can be difficult to generate in weak patients (e.g. stroke, spinal cord injury). 
Section: Toward Muscle-Driven Control of Wearable Robots: a Real-Time Framework for the Estimation of Neuromuscular States During HumanExoskeleton Locomotion Tasks

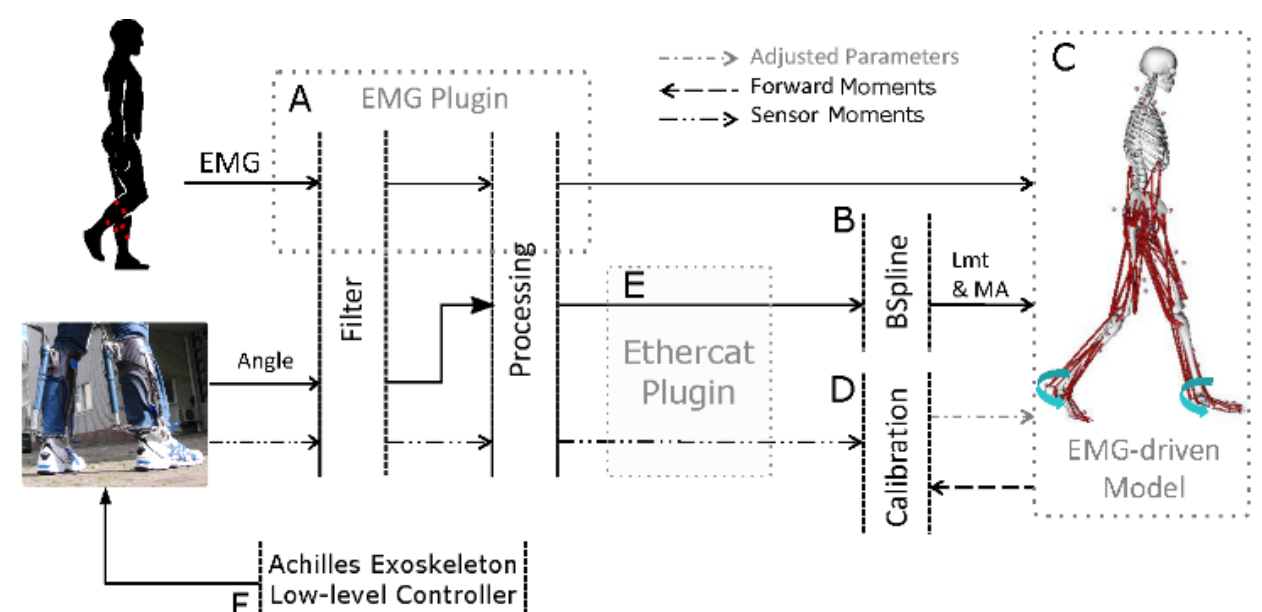

Figure 3-1: Schematic representation of the framework and its interface with the Achilles ankle exoskeleton. See methods section for explanation of the individual components.

Further approaches rely on electromyography (EMG) signal to control exoskeletons. For example in [77], EMG amplitude is used as a proportional control input to a pneumatic actuator. Even if this gives access to the user's intention it ignores the mechanical force contribution of the user, which in general does not vary linearly with EMG amplitude [71]. The work in [78] used muscle activation primitives derived from static optimization along with an oscillator gait phase detector and a fuzzy-logic classifier for task recognition to control a neuromusculoskeletal (NMS) model. This provides a step toward more natural assistance but still lacks to integrate human bio-electrical signals in the control loop. This limits the ability to account for an individual's actual muscle activation patterns and forcegenerating capacity with limited applicability to patients [17].

To enable optimal and personalized human-machine physical interaction we propose the development of control schemes that can close the loop at the human muscle level. This would enable optimal synchronization between human muscular contraction and device actuation, enabling the wearable robot to become a natural extension of the own body.

This paper presents a computational framework for the real-time estimation of human neuromuscular states during a repertoire of human-exoskeleton locomotion tasks. This allows real-time recording and processing of neural surrogates, i.e. EMG-derived neural activations to muscles. Moreover, it enables creating subject-specific physiologically correct computational models of the musculoskeletal system. Overall this allows for the real-time simulation of the human musculoskeletal dynamics as controlled by incoming neural activations recorded in vivo. Knowing this neural surrogate and internal muscle parameters allows our framework to extrapolate outside of the task used for model calibration [79]. We tested this framework on an individual wearing the bi-lateral ankle exoskeleton Achilles [7]. 
We show how the framework can be used to determine how different exoskeleton assistive modes contribute to alter the human muscle behavior, something central for the development of future symbiotic controllers. We also show how the current framework operates in real-time within the human muscle electromechanical delay, i.e. this deadline within an HMI would ensure the assistive device to be actuated synchronously with the user's muscle mechanical function.

\subsection{Methods}

To open a window into human neuromuscular states, we created a real-time EMG-driven musculoskeletal modelling framework [14], [80]. This is based on the algorithm developed in [14] and later enhanced to work in real-time [79]. We interfaced the framework with the Achilles robotic exoskeleton, i.e. a bi-lateral orthosis powered via series-elastic actuation to support plantar-flexion [7]. A schematic representation of the framework and his interface with the Achilles is presented in Fig. 1.

The EMG-driven musculoskeletal model (Fig. 1C) used is made of four degrees of freedom (DOFs), i.e. bi-lateral knee flexion-extension and ankle plantar-dorsiflexion. The model also includes 14 musculo-tendon unit (MTU) consisting of the Gastrocnemius Medialis right and left, Gastrocnemius Lateralis right and left, Soleus right and left, Tibialis Anterior right and left, Peroneus Brevis right and left, Peroneus Longus right and left and Peroneus Tertius right and left.

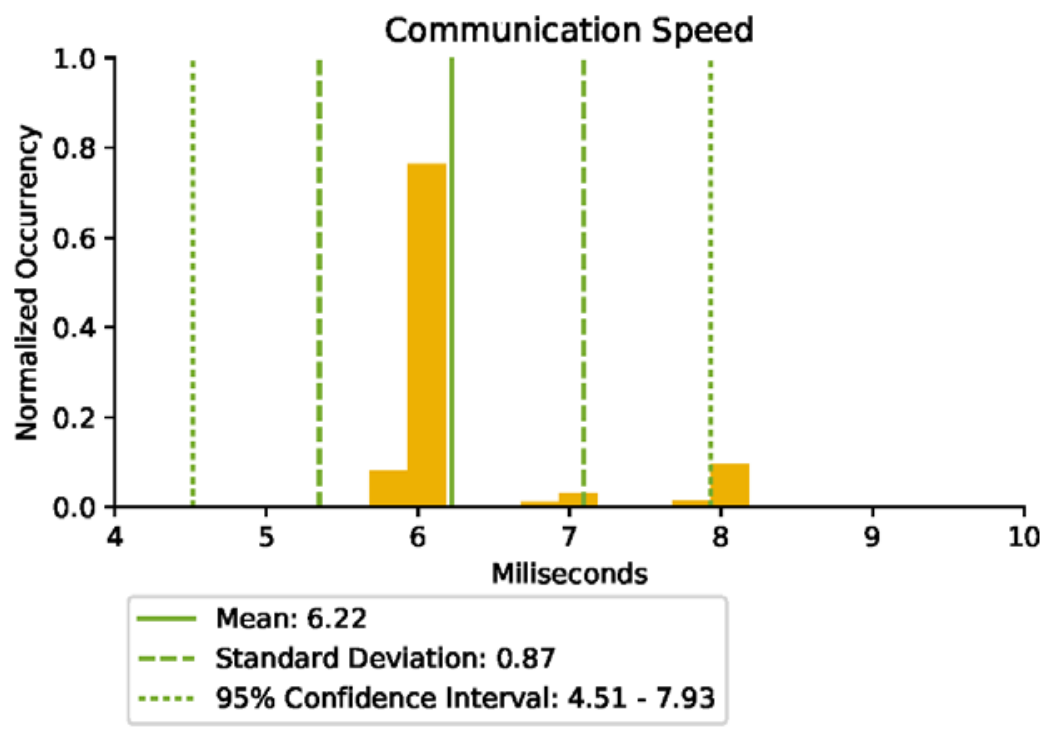

Figure 3-2: Bar graph of the transfer time to send and receive information between the EMG-driven framework and the Achilles low level controller. 
Section: Toward Muscle-Driven Control of Wearable Robots: a Real-Time Framework for the Estimation of Neuromuscular States During HumanExoskeleton Locomotion Tasks
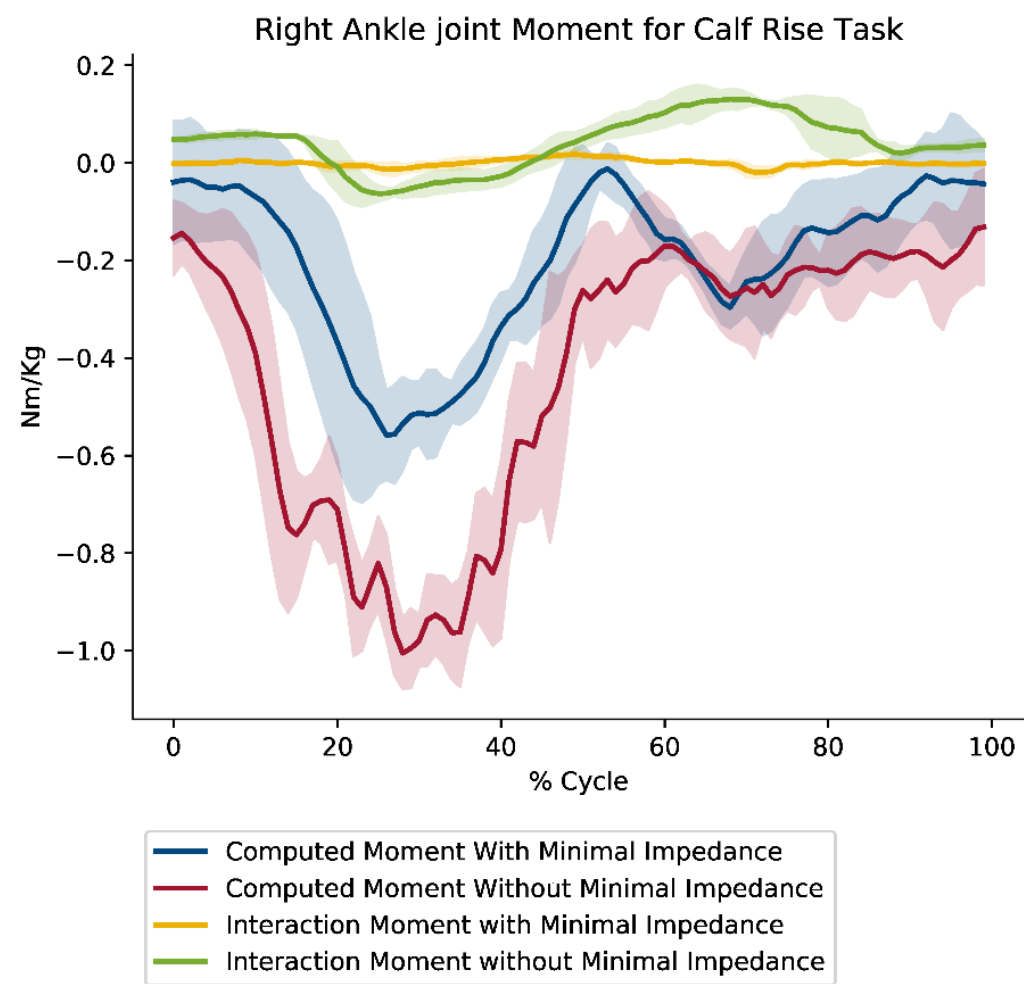

Figure 3-3: Mean computed moments in real-time by the EMG framework and mean interaction moment recorded during calf rise for the right ankle for all repetition.

The EMG-driven model receives two inputs; I) EMG recordings and II) user's ankle joint kinematics. We developed a plug-in to record in real-time EMGs signals from 10 muscle groups (Fig 1A) including: Gastrocnemius Lateral and Medialis right and left, Soleus right and left, Tibialis Anterior right and left and Peroneus Longus right and left. This was done using a wireless Delsys Trigno system (Delsys inc., Massachusetts, USA). Each recorded muscle EMG was transmitted to its respective controlled MTU in the model. That is, the Peroneus Longus EMG controlled the Peroneus Longus, Peroneus Brevis and Peroneus Tertius MTUs in the model, the Tibialis Anterior EMG controlled the Tibialis Anterior MTU, the Soleus EMG controlled the Soleus MTU, the Gastrocnemius Medialis EMG controlled the Gastrocnemius Medialis MTU and the Gastrocnemius Lateralis controlled the Gastrocnemius Lateralis MTU. EMGs were recorded at a frequency of $2000 \mathrm{~Hz}$, then high pass filtered at $20 \mathrm{~Hz}$, rectified, low pass filtered at $6 \mathrm{~Hz}$ and normalized using maximal EMG recorded during maximum voluntary contraction (MVC). 


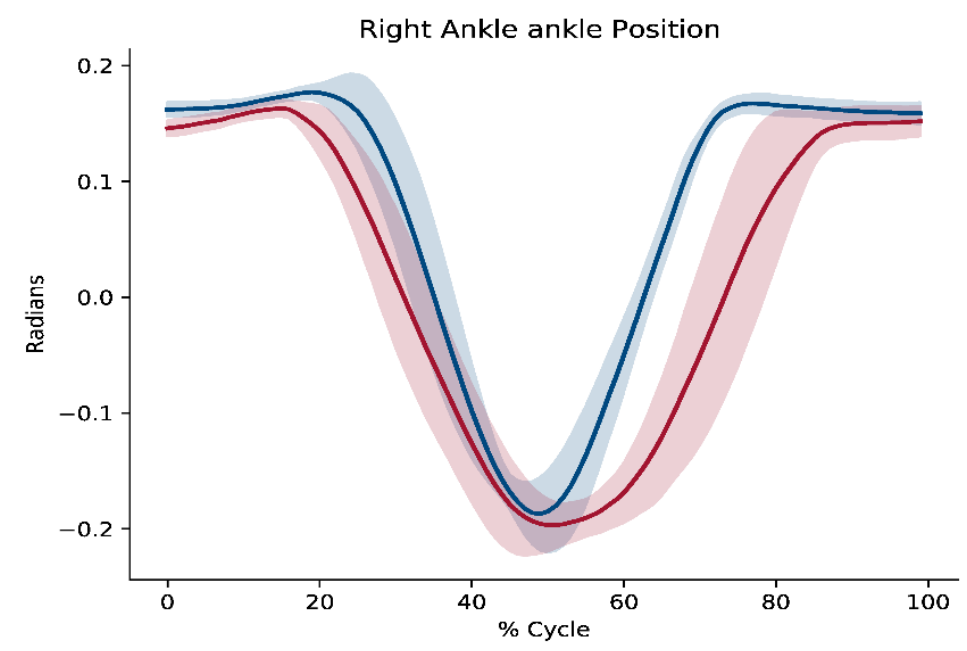

With Minimal Impedance

Without Minimal Impedance

Figure 3-4: Mean position of the right ankle during calf rise over all repetition.

Ankle joint kinematics were recorded in real-time from the Achilles ankle exoskeleton angle encoders. This information was subsequently used to estimate musculotendon kinematics, i.e. MTU length (LMT) and moment arms (MA) [21]. Our previously proposed B-Spline algorithm was used to synthetize the subject-specific musculoskeletal model in a set of multidimensional spline function and convert joint angles into smooth LMT and MA online (Fig. 1B) [79] [21]. EMG recordings and MTU kinematics were used by the musculoskeletal model to compute the interplay between muscle fibers and series tendons. This is done via a computationally efficient implementation of the Hill-type muscle model [16], [55] providing online estimates of variables including: muscle force, fiber length, fiber velocity and pennation angle and also joints variables such as joints moments.

To obtain output from the EMG-driven model matching a specific user, the model first needs to be scaled to match individual anthropomorphic properties. Then, muscle parameters need to be personalized to the user. For this, an optimization-based calibration procedure is used (Fig. 1D). We first pre-scale tendon slack length and optimal fiber length using the method described in [56]. We then use an optimization procedure to minimize the error between the moment computed by our framework and experimental moment obtained by the Achilles moment sensor by varying muscle parameters (i.e. tendon slack length, optimal fiber length, maximal isometric force and EMG-to-activation shape factor). During this calibration, the B-Spline coefficients are also computed.

The real-time interface with the Achilles exoskeleton was done using the Ethernet for Control Automation Technology (EtherCAT) communication protocol. The EtherCAT plugin uses the TwinCAT software (Beckhoff Automation GmbH \& Co. KG, Germany) 
Section: Toward Muscle-Driven Control of Wearable Robots: a Real-Time Framework for the Estimation of Neuromuscular States During HumanExoskeleton Locomotion Tasks

and an EtherCAT PCI slave card (FC1100, Beckhoff Automation GmbH \& Co. KG, Germany) to communicate in hard real-time with the Achilles ankle exoskeleton. Furthermore, the plugin is sending information to the framework using the Automation Device Specification (ADS) protocol. The plugin sends right and left ankle position and right and left ankle interaction moment to the framework (Fig. 1E).

The Achilles exoskeleton is an autonomous ankle exoskeleton. The exoskeleton is actuated by a series elastic actuator, where a Maxon ECC22 motor (Maxon Motor AG, Switzerland) with a SH6x2 ball-screw gear (SKF, Sweden) moves a custom designed leaf spring. The exoskeleton is controlled from a computer running Linux in a backpack. Simulink (MathWorks, US) is used for the high-level control, which interfaces with EtherCAT that controls the motor drive, an EPOS3 70/10 EtherCAT (Maxon Motor, Switzerland). The Achilles exoskeleton is controlled as follows. The desired reference moment is converted to a reference position of the ball screw. This position is then converted to a target velocity that is sent to the drive. Therefore, a filtered derivative of the position is added to the unfiltered position error. In the drive (EPOS3), the signal is converted to a motor current using two cascaded PI controllers. The EMG-driven framework and the TwinCat software were

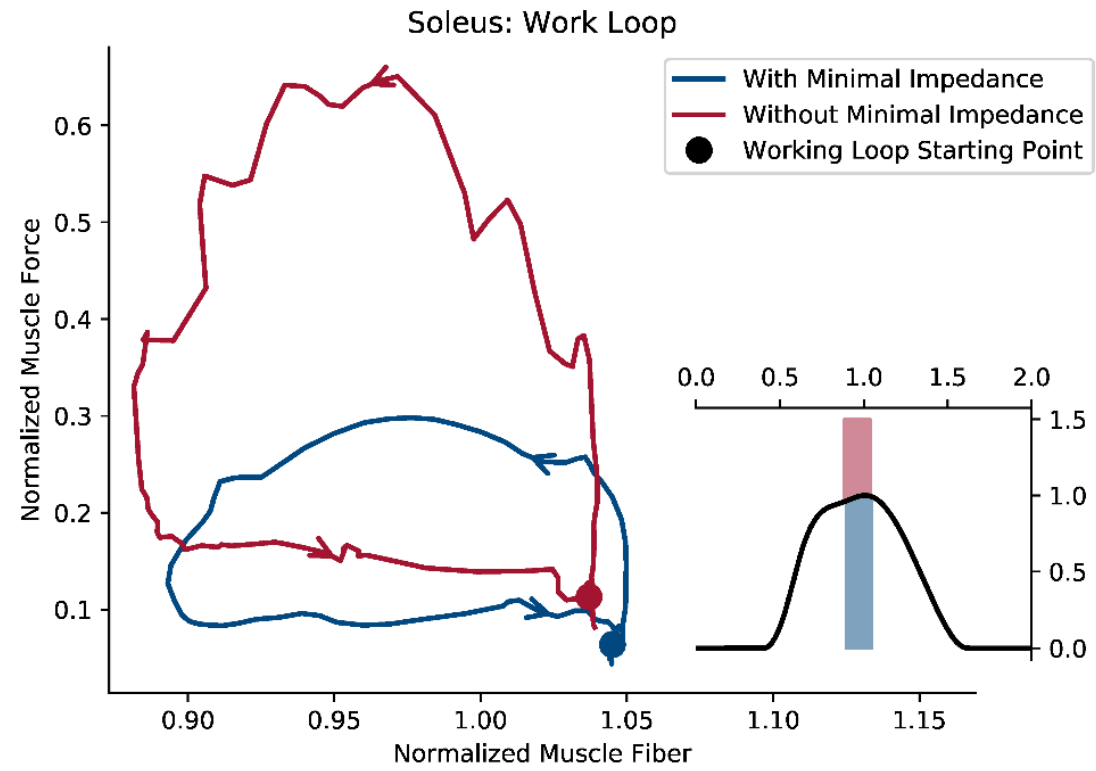

Figure 3-5: Work loop of the right soleus muscle during calf rise and the projection of the work space of the normalized fiber length on the active fiber force-length relationship curve (subplot). The $\mathrm{Y}$ axis represents the mean muscle force and the $\mathrm{X}$ axis represented represent the normalized fiber length (i.e. fiber length divided by optimal fiber length). 
operated on a desktop computer (Intel i7. 3.4 GHz (Intel, Santa Clara, CA, US), 12 Gb RAM, Windows 7) with the PCI EtherCat slave card installed on it. This computer was connected to the Achilles computer (NUC computer, Intel i3 1.8 GHz (Intel, Santa Clara, CA, US), 16 Gb RAM, Linux) via an Ethernet cable.

\subsection{Experiments}

To test the interface and the framework, we conducted experiments with one healthy subject (male, $73 \mathrm{Kg}, 28$ years old, $177 \mathrm{~cm}$ ). EMG sensors were first placed on the ankle of the subject with respect to the muscles area. After placement, MVCs were performed for the normalization of the EMG during the rest of the experiment. Once MVC realized, the subject was asked to wear the Achilles ankle exoskeleton. The exoskeleton was then turned on and locked in position at an angle of around 90 degrees for both legs, for the rest of the experiment the Achilles was unlocked. The subject was then asked to realize isometric plantar dorsiflexion against the exoskeleton. These allow us to record isometric moments using the interaction moments sensor. This recording was used to calibrate the model. After the calibration, which takes less than 10 minutes, different locomotion tasks were realized with the EMG driven model framework computing in real-time joint moments and internal muscles parameters. The first task was to realize a series of calf rises. The second task was to

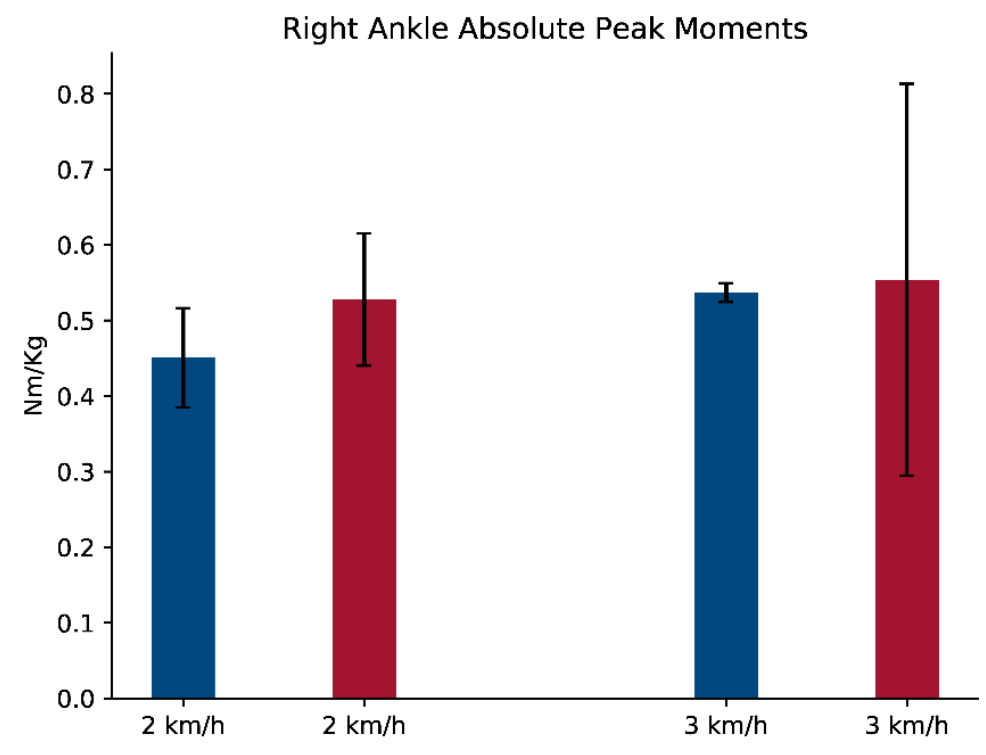

With Minimal Impedance

Without Minimal Impedance

Figure 3-6: Peak of the absolute value of the computed moment for the gait task on treadmill for 2 $\mathrm{km} / \mathrm{h}$ and $3 \mathrm{~km} / \mathrm{h}$ and two conditions, with and without minimal impedance. 
Section: Toward Muscle-Driven Control of Wearable Robots: a Real-Time Framework for the Estimation of Neuromuscular States During HumanExoskeleton Locomotion Tasks

walk on a treadmill with a speed of $2 \mathrm{~km} / \mathrm{h}$ and $3 \mathrm{~km} / \mathrm{h}$. The last task was to realize overground locomotion directly followed by a stair ascension without pause during the two. Every task was realized with two exoskeleton operating modes: I) with the minimal impedance control and II) with motor off, thereby having the passive compliance of the motor acting on the human limb.

An instance of the experiments can be seen in Movie 1. Across all experiments, we quantified communication speed between the EMG-driven framework and the low-level controller of the Achilles exoskeleton. The time between the sending of the information from the framework and the receiving of the same information resend by the low-level controller was recorded.

\subsection{Results}

The first test reports bidirectional communication speed between the exoskeleton and computer unit (Fig. 2). The mean speed was $6.22 \pm 0.87 \mathrm{~ms}$ with a $95 \%$ confidence interval (two standard deviations) comprised between 4.51 and 7.93 ms. Speeds estimates were computed over 60000 data samples.

The second test reports both ankle joint sagittal position recorded via exoskeleton encoders (Fig. 3), as well as the ankle plantar-dorsiflexion moment, computed in real-time by our framework (Fig. 4) during calf rise tasks. Fig. 3 shows that both exoskeleton assistance modes underlie similar ranges of motion ( $0.34 \mathrm{rad}$, with $-0.1 \mathrm{rad}$ offset difference) but different slopes (affecting task average speed). For the right ankle position the maximal angle was $0.17 \pm 0.006 \mathrm{rad}$ and minimal angle $-0.18 \pm 0.03 \mathrm{rad}$ with minimal impedance and $0.16 \pm 0.007 \mathrm{rad}$ for maximal angle and $-0.19 \pm 0.022 \mathrm{rad}$ for a minimal angle without minimal impedance. Fig. 4 shows a pronounced change of peak moments across exoskeleton assistance modes. That is, that both human ankle moment and human-exoskeleton interaction moment were smaller using the minimal impedance controller. This is confirmed by the root means square (RMS) of the moments with a value of $0.26 \mathrm{Nm} / \mathrm{Kg}$ with minimal impedance and $0.007 \mathrm{Nm} / \mathrm{Kg}$ for the interaction moment and $0.51 \mathrm{Nm} / \mathrm{Kg}$ without minimal impedance and $0.068 \mathrm{Nm} / \mathrm{Kg}$ for the interaction moment.

The third test provided access to muscle behavior. This was reported at the level of the force-length work loop for the soleus muscle during calf rise (Fig. 5). Exoskeleton assistance modes resulted in substantial modulation of the work-loop specifically pronounced on the force axis. The minimum normalized muscle force was 0.04 with minimal impedance and 0.08 without minimal impedance, the maximum normalized muscle force is 0.29 with minimal impedance and 0.65 without minimal impedance. The minimal normalized fiber length is 0.89 with minimal impedance and 0.88 without it, the maximal normalized length is 1.05 with minimal impedance and 1.04 without it. Fig. 5 also shows that the operating 
length range of the muscle was not altered across modes with the Soleus short-stretch cycles always centered around the plateau of the force-length relationship.

The fourth test reported variation of the peak moment during gait at $2 \mathrm{~km} / \mathrm{h}$ and $3 \mathrm{~km} / \mathrm{h}$ (Fig. 6) as well as the peak muscle force during the same conditions (Fig. 7). In Fig. 6 , we can see that the user developed more moment when minimal impedance was not in use. The mean peak force developed during the gait at $2 \mathrm{~km} / \mathrm{h}$ with minimal impedance is $0.45 \pm 0.065 \mathrm{Nm} / \mathrm{Kg}$ and $0.52 \pm 0.08 \mathrm{Nm} / \mathrm{Kg}$ for $3 \mathrm{~km} / \mathrm{h}$, without minimal impedance it was $0.53 \pm 0.012 \mathrm{Nm} / \mathrm{Kg}$ for $2 \mathrm{~km} / \mathrm{h}$ and $0.55 \pm 0.26 \mathrm{Nm} / \mathrm{Kg}$ for $3 \mathrm{~km} / \mathrm{h}$. The cumulative (i.e. of all right leg muscles considered by the model) mean normalized force (Fig. 7) is with minimal impedance 1.35 for $2 \mathrm{~km} / \mathrm{h}$ and 1.47 for $3 \mathrm{~km} / \mathrm{h}$ and 1.64 for $2 \mathrm{~km} / \mathrm{h}$ and 1.52 for $3 \mathrm{~km} / \mathrm{h}$ without minimal impedance. The biggest contributor was the Gastrocnemius Medialis when normalized but the Tibialis Anterior was the biggest contributor in net forces in newton.

The fifth test reported ankle muscles moment in the transition between gait to stair ascending task (Fig. 8). We can see the muscles moment computation continuity from start to stop and an increase in moments for plantar dorsiflexion muscles during stair ascending. In Movie 1, the different task can be visualized.

\subsection{Discussion}

The motivations behind this study were I) to develop a real-time version of our framework directly interfaced with a bilateral ankle exoskeleton, II) to have a proof of concept experiment that tests whether the framework can be applied over a range of locomotion tasks and III) to capture human-exoskeleton interaction at the level of muscle mechanics and how this is altered by exoskeleton assistive modes.

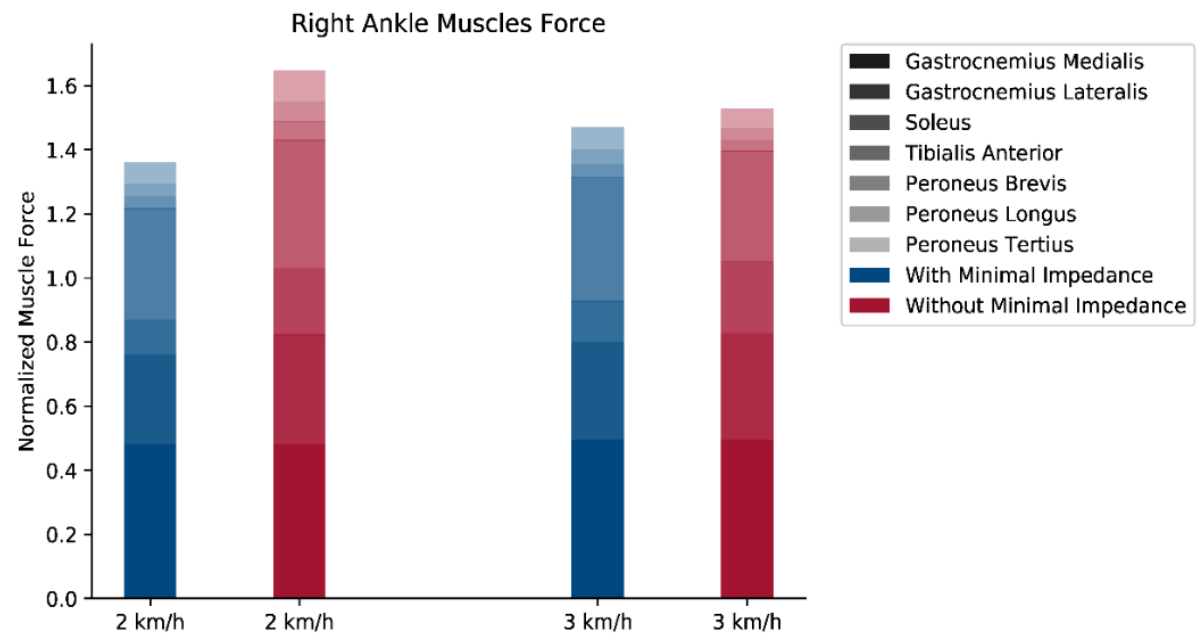

Figure 3-7: Maximal forces for all muscles for the right ankle considered in the model during the gait task for $2 \mathrm{Km} / \mathrm{h}$ and $3 \mathrm{Km} / \mathrm{h}$ and two conditions, with and without minimal impedance. 
Section: Toward Muscle-Driven Control of Wearable Robots: a Real-Time Framework for the Estimation of Neuromuscular States During HumanExoskeleton Locomotion Tasks
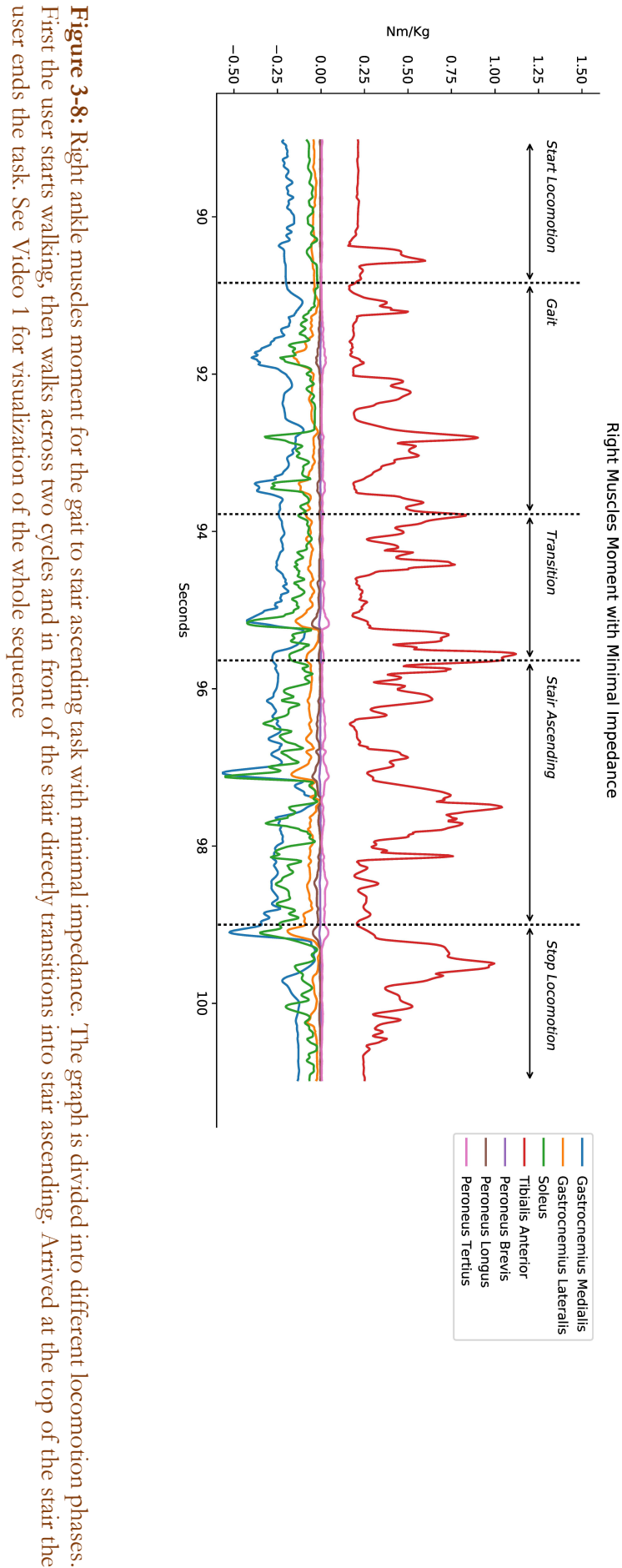
These steps are central toward the creation of novel task-independent controllers that can operate in symbiosis with the operator's muscle and provide optimal assistance.

Current HMIs using bio-electrical signals (i.e. EMG) do not fully account for the form and function of the human musculoskeletal system, thus limiting the ability to optimally support an individual based on residual force-generating capacity. Other HMIs based only on mechanical inputs are efficient for a specific task, but do not easily generalize to other tasks [6], [30] or do not provide a neuromuscular benefit [76], [78] (i.e. no metabolic reduction). Furthermore, these HMIs do not provide direct muscle level understanding of how they affect the wearer [81]. This article shows the ability to account for both the actual muscle activation (via EMG) and the user's force-generating properties (via subject-specific modelling).

Our results showed that our framework could run in real-time with a computation time always lower than $11 \mathrm{~ms}$ (i.e. Fig. 2). Having a framework with the smallest delay possible is important as a desynchronization between received moment from an exoskeleton and the wearer can increase metabolic consumption [30]. Results also showed the impact of exoskeleton assistance modes on the joint moment generation capacity (Fig. 4). In this, peak moment reductions result from the Achilles exoskeleton absorbing the gravitational contribution of the ankle moment. Fig. 5 shows that our proposed signal-driven modelling approach opens a window into muscle level mechanics when wearing the Achilles exoskeleton. This is central to understand the effect that the exoskeleton can have, positive or negative, on the wearer. Computed work-loops for the Soleus show that the muscle fibers operate on length ranges centrally located toward the optimal plateau (i.e. around 1) of force production in the active force fiber length relationship curve when minimal impedance is used. Achieving this type of measurement would normally involve long and offline experimental procedures using expensive ultrasound and dynamometer technology. Here we show that muscle mechanics estimates can be obtained in real-time using a fully wearable robot. The same reasoning can also be made for metabolic consumption, as muscle modelling can also compute metabolic energy costs [82], which can remove the need for expensive metabolimeter systems and give immediate result in real-time. Our results showed that moment reduction between minimal impedance and without minimal impedance (Fig. 6) are more important at a walking speed of $2 \mathrm{Km} / \mathrm{h}(\approx 0.07 \mathrm{Nm} / \mathrm{Kg})$ than at $3 \mathrm{Km} / \mathrm{h}(\approx$ $0.02 \mathrm{Nm} / \mathrm{Kg}$ ). Moreover, these can be computed continuously as well as during transitions across motor tasks (Fig. 8). This offers an important opportunity for exoskeleton HMIs than conventional state machines.

This study has limitations. The calibration procedure needs further experimental validation, i.e. via ultrasonography for muscle parameters or intramuscular pressure sensing for muscle force. Further, the transmission of mechanical moments from the exoskeleton to the biological ankle joint may be affected by joint misalignment. Composite human-exoskeleton models need to be developed to account for these factors. 
Section: Toward Muscle-Driven Control of Wearable Robots: a Real-Time Framework for the Estimation of Neuromuscular States During HumanExoskeleton Locomotion Tasks

\subsection{Conclusion}

We have shown the possibility to have a muscle-level understanding of human-exoskeleton interaction during different locomotion tasks. We also showed that we can have a real-time interface between our framework and the Achilles ankle exoskeleton. This will open new avenues for the creation of symbiotic exoskeleton and understanding the effect of exoskeleton on users. 


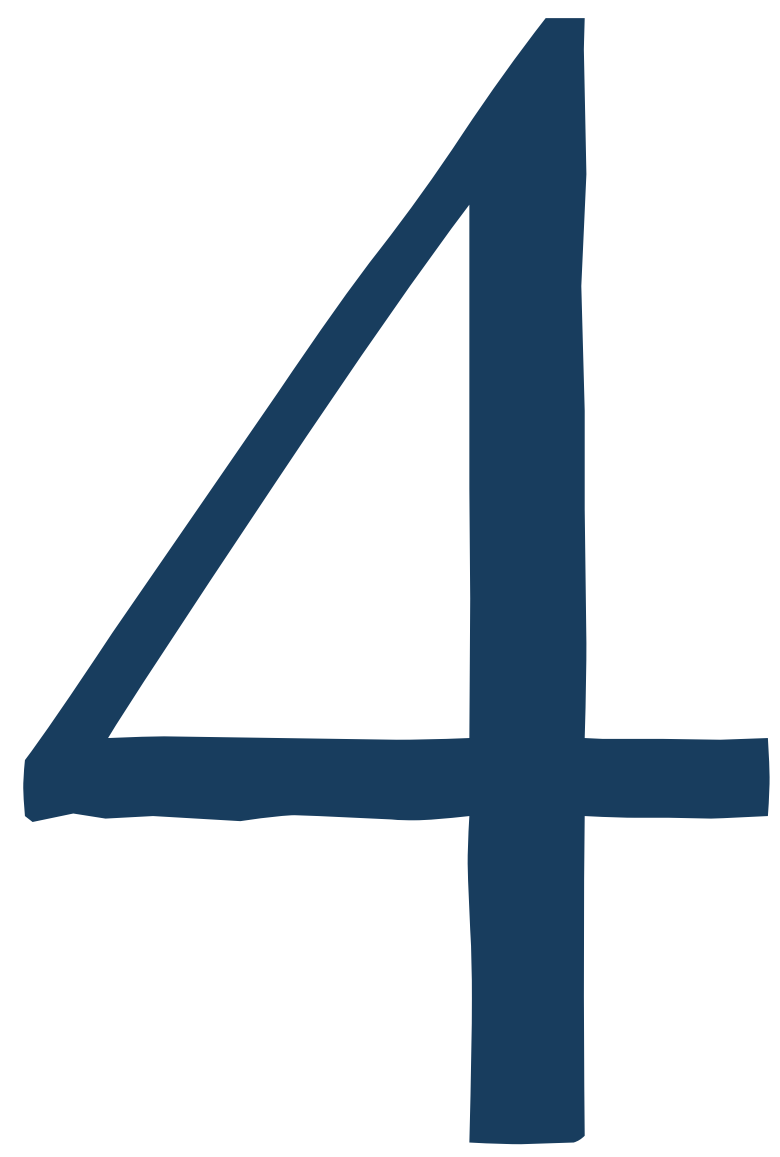




\title{
VOLUNTARY CONTROL OF WEARABLE ROBOTIC EXOSKELETONS BY PATIENTS WITH PARESIS VIA NEUROMECHANICAL MODELING
}

\section{Guillaume Durandau, Dario Farina, Guillermo Asin-Prieto, Iris Dimbwadyo-Terrer, Sergio Lerma- Lara, Jose L. Pons, Juan C. Moreno, Massimo Sartori}

\begin{abstract}
Research efforts in neurorehabilitation technologies have been directed towards creating robotic exoskeletons to restore motor function in impaired individuals. However, despite advances in mechatronics and bioelectrical signal processing, current robotic exoskeletons have had only modest clinical impact. A major limitation is the inability to enable exoskeleton voluntary control in neurologically impaired individuals. This impacts the possibility of optimally inducing the activity-driven neuroplastic changes that are required for recovery. We have developed a patient-specific computational model of the human musculoskeletal system controlled via neural surrogates, i.e., electromyography-derived muscle neural activation surrogates. The electromyography-driven musculoskeletal model was synthesized into a human-machine interface (HMI) that enabled poststroke and incomplete spinal cord injury patients to voluntarily control multiple joints in a multifunctional robotic exoskeleton in real-time. We demonstrated patients' control accuracy across a wide range of lower-extremity motor tasks. Remarkably, an increased level of assistance from the exoskeleton always resulted in a reduction in both amplitude and variability of muscle activations as well as in the mechanical moments required to perform a motor task. Since small discrepancies in onset time between human limb movement and that of the parallel exoskeleton would potentially increase human neuromuscular effort, these results demonstrate that the developed HMI precisely synchronizes the device actuation with the residual voluntary muscle contraction capacity in neurologically impaired patients. Continuous voluntary control of robotic exoskeletons (i.e. event-free and task-independent) has never been demonstrated before in populations with paretic and spastic-like muscle activity, such as those investigated in this study. Our proposed methodology may open new avenues for harnessing residual neuromuscular function in neurologically impaired individuals via symbiotic wearable robots.
\end{abstract}


Section: Voluntary Control of Wearable Robotic Exoskeletons by Patients with Paresis via Neuromechanical Modeling

Keywords - electromyography; EMG-driven modeling; neuromechanical modeling; neuromuscular injury; robotic exoskeleton.

Publication-Durandau, G., Farina, D., Asín-Prieto, G. et al. J NeuroEngineering Rehabil (2019) 16: 91. 


\subsection{Introduction}

The ability to walk directly relates to the quality of life. Neurological lesions such as those underlying stroke and spinal cord injury (SCI) often result in severe motor impairments (i.e., paresis, spasticity, abnormal joint couplings) that compromise an individual's motor capacity and health throughout the life span. For several decades, scientific effort in rehabilitation robotics has been directed towards exoskeletons that can help enhance motor capacity in neurologically impaired individuals. However, despite advances in mechatronics and bioelectrical signal processing, current robotic exoskeletons have had limited performance when tested in healthy individuals [30] and have achieved only modest clinical impact in neurologically impaired patients [83], e.g., stroke [84], [85], SCI patients [86].

Two major challenges are hampering progress. The first is the inability of current systems to enable an individual patient to voluntarily control the robotic device while inducing a positive modulation of neuromuscular activity. This prevents wearable robots from optimally inducing the activity-driven neuroplastic changes that are required for recovery [87], [88]. The second is an incomplete understanding of how lesions in the central nervous system (CNS) impact musculoskeletal system function, which impedes understanding how patients' motor intentions should be best supported by a robotic device.

Following a brain lesion, secondary adaptation processes occur in the entire musculoskeletal system, i.e., alterations of muscles, ligaments and tendons properties [89]. In stroke survivors, this results in stiffness and disruption of muscle tone [90] followed by abnormal muscle contractile dynamics and consequent changes in locomotion control paradigms [83]. The scarcity of knowledge regarding the adaptation mechanisms taking place in the composite neuromusculoskeletal system has limited our ability to understand what drives impairment and therefore how to restore lost motor capacity via wearable robots. The development of human-machine interfaces (HMIs) that can take into account individual patients' neuromuscular alterations is fundamental for enhancing the motor function of neurologically impaired patients [88].

HMIs in commercially available robotic exoskeletons for neurorehabilitation (e.g., Rewalk [91], Lokomat [92] and LOPES [75]) largely rely on position and impedance control [93], [94]. In these approaches, the robotic exoskeleton creates joint trajectories or force fields along predefined trajectories previously extracted from healthy populations [32], [95]. However, this does not fully engage the patient, limiting the emergence of positive neuroplasticity [96], with limited rehabilitation outcomes with respect to conventional therapy [85], [86]. Noncommercial robotic exoskeletons use more complex schemes including HMIs inspired by mechanical principles, e.g., predefined moment patterns triggered at specific phases of the gait cycle [6], [97], [98]. These approaches were recently applied to poststroke and cerebral palsy individuals [97], [99], [100]. However, such approaches are limited to supporting a cyclic gait under specific patterns and speeds, thus 
limiting the patient's self-pacing and voluntary control of the exoskeleton. A generalization of these methods was proposed via human-in-the-loop paradigms where moment patterns for the exoskeleton are optimized online to reduce the metabolic cost of locomotion [30]. Human-in-the-loop optimization, however, operates on large time scales. That is, for the wearable robot to react and adapt to movement variations, the controller needs to process several minutes of metabolic data (i.e., > 20 minutes), limiting current applications to healthy individuals only. Alternative approaches use sensitivity amplification control algorithms, where exoskeleton sensory information (i.e., interaction forces) is used to generate control commands [76], [101]. However, this paradigm does not provide support until the patient has produced detectable mechanical force or movement, thus critical in severely impaired individuals [17]. As a result, this has not been employed in patients to provide neuromuscular effort reduction [102].

Other HMI schemes rely on bioelectrical signals recorded from muscles or brain areas [103]. These methods could potentially enable exoskeletons to promptly respond and adapt to the patient's motor intention, a central aspect of neurorehabilitation robotics [17], [83], [88]. Current schemes include neuro-fuzzy approaches [104] or proportional myocontrol methods [105]-[107] that use electromyograms (EMGs), sometimes in conjunction with foot-ground reaction forces, to generate direct control commands. However, these methods do not account for the nonlinearity between EMG amplitude and muscle mechanical force, the effect of which is especially important for the rehabilitation of neurologically impaired individuals [108]-[110]. As a result, these methods would not always enable optimal computation of exoskeleton assistive moments proportionally to the patient's forcegenerating capacity [71], [111]. This ultimately hinders patient-machine synchronization and limits the patient's ability to control the exoskeleton voluntarily. Moreover, methods based on proportional myocontrol and foot-ground reaction forces [105], [106] are designed for cyclic locomotion where the subject receives support during a specific part of the gait cycle. However, these methods rely on detection of pre-defined gait events (e.g. foot-ground contact) and are tuned for a specific motor task (e.g. ground-level locomotion) and joint. Overall, this does enable continuous (event-free and task-independent) control of robotic exoskeletons. Alternative bioelectrical signals such as electroencephalographs [112]-[115] are currently limited in the context of robotic exoskeletons due to the high sensitivity of the signal to movement artefacts [74].

We have developed an HMI based on EMG-driven musculoskeletal modelling. This approach accounts for the form and function of the human neuromusculoskeletal system in neurologically impaired patients with paresis. We tested it in a wheelchair-bound patient with incomplete SCI and in two chronic hemiparetic post-stroke survivors with residual walking capabilities. Although EMG-driven musculoskeletal modelling was previously employed in conjunction with robotic devices [18], [23], [24] it was never applied to neurologically 


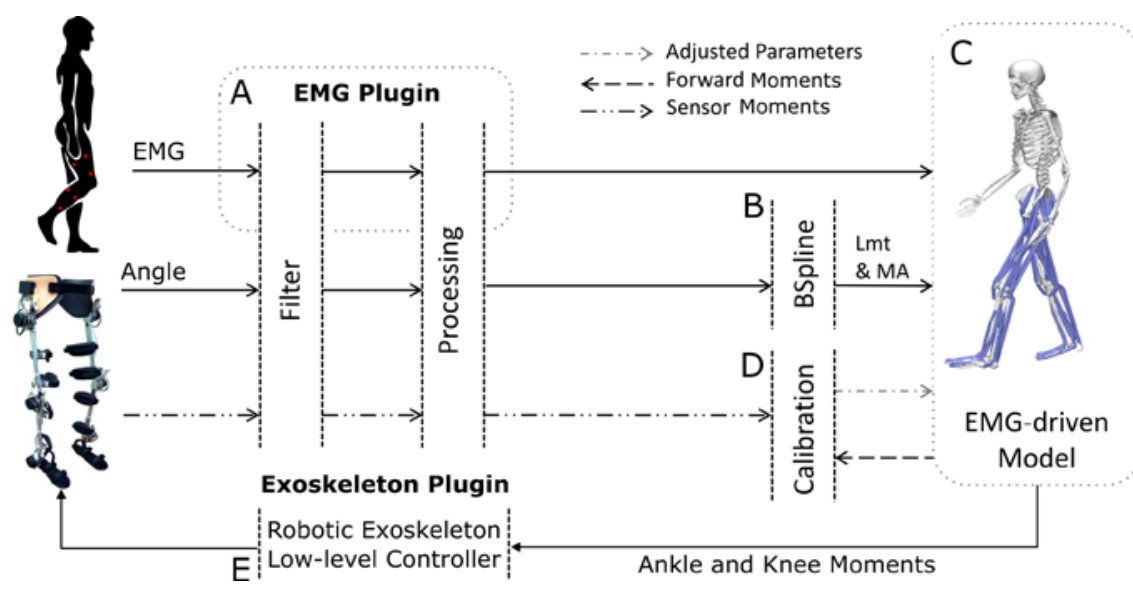

Figure 4-1: Schematic representation of the real-time modeling framework and its communication with the robotic exoskeleton. The whole framework is operated by a Raspberry Pi 3 single-board computer. The framework consists of five main components: (A) The EMG plugin collects muscle bioelectric signals from wearable active electrodes and transfers them to the EMG-driven model. (B) The B-spline component computes musculotendon length (Lmt) and moment arm (MA) values from joint angles collected via robotic exoskeleton sensors. (C) The EMG-driven model uses input EMG, Lmt and MA data to compute the resulting mechanical forces in 12 lower-extremity musculotendon units (Table 1) and joint moment about the degrees of freedom of knee flexionextension and ankle plantar flexion-dorsiflexion. (D) The offline calibration procedure identifies internal parameters of the model that vary non-linearly across individuals. These include optimal fiber length and tendon slack length, muscle maximal isometric force, and excitation-to-activation shape factors. (E) The exoskeleton plugin converts EMG-driven model-based joint moment estimates into exoskeleton control commands. Please refer to the Material and Methods section for an in-depth description.

impaired individuals to demonstrate neuromuscular activity reduction. To the best of our knowledge, our results demonstrate the first model-based HMI that enables neurologically impaired patients with paresis to voluntarily control multiple degrees of freedoms (DOFs) in complex robotic exoskeletons. Importantly, the results demonstrate that increased levels of exoskeleton assistance induced a positive modulation of neuromuscular activity across a large repertoire of motor tasks. This was reflected in a reduction in both the amplitude and the variability of muscle activations as well as in the resulting human joint moments required to perform a motor task. Since small discrepancies in onset time between human limb movement and that of the parallel exoskeleton can significantly increase human muscle effort [30], our results demonstrate that the proposed approach can precisely synchronize device actuation with human muscle contraction, which is especially challenging in pathological populations with paretic and spastic-like muscle activity.

With neurorehabilitation in mind, it is important stressing that the goal of our HMI is not that of reducing the operator's EMGs per se. The goal is rather that of amplifying the subject's force-generating capacity to enable the mechanical moments necessary to execute motor tasks that could not otherwise be performed without the support of the exoskeleton. 
The overarching goal of the experiments presented in this paper was to enable neurologically impaired patients to voluntarily control a multi-DOF robotic exoskeleton while receiving positive physical assistance [32]. In particular, the major objectives of this study were to test whether I) both healthy subjects and neurologically impaired patients could voluntarily control the angular position of the exoskeleton multiple joints accurately and II) whether our proposed framework could modulate the neuromuscular activity of healthy subjects and neurologically impaired patients (i.e., their muscle activations and resulting moments) as a function of different exoskeleton assistance levels with no loss of joint control accuracy.

\subsection{Methods}

We developed a computational patient-specific model of the human lower-extremity musculoskeletal system (Fig. 1). This enabled estimating the mechanical force produced in 12 lower-extremity musculotendon units (MTUs, Table 1) as well as the resulting moments about knee flexion-extension and ankle plantar-dorsiflexion DOFs. Subject-specific models were built individually for four healthy individuals, one incomplete SCI patient and two hemiparetic stroke patients (Table 2).

We demonstrated the feasibility of using real-time model-based joint moment estimates for the voluntary control of a robotic exoskeleton throughout a large repertoire of ankle-knee motor tasks. Our proposed framework schematic is depicted in Fig. 1. First, we describe the computational modelling framework structure. Then, we describe the movement data recording and testing protocol.

\subsubsection{Computational modelling framework}

EMG-driven musculoskeletal modelling framework: We developed an online framework that computes joint moments from EMG signals based on our previous works [14], [51], [79]. The framework comprises five main components (Fig. 1). The EMG plugin component (Fig. 1A) provides a direct TCP/IP connection with the external EMG system. It records EMGs produced by active electrodes and sends them to the EMG-driven model component.

The exoskeleton plugin component (Fig. 1B) records exoskeleton sensory information including human-exoskeleton interaction moments via strain gauges placed on every DOF, as well as the motor moments recorded via motor currents. This plugin assures the transmission of joint moments estimated via the EMG-driven model to the robotic exoskeleton.

The musculotendon kinematics component (Fig. 1C) synthesizes MTU paths defined in the subject-specific geometry model (see Experimental Procedures Section below) into a set of MTU-specific multidimensional cubic B-splines. Each B-spline computes MTU length and moment arms as a function of input joint angles [21]. It enables fast and accurate 
computation of smooth MTU kinematics, which is central for subsequent moment calculation [21].

The EMG-driven model component (Fig. 1C) converts eight input EMG signals into neural activation for 12 MTUs, as presented in Table 1. This is done using a 2nd-order twitch model (eq. 1) and a nonlinear transfer function (eq. 2) [71], [79]:

$$
\begin{aligned}
& u_{j}(t)=\alpha e_{j}(t-d)-\beta_{1} u_{j}(t-1)-\beta_{2} u_{j}(t-2) \\
& a_{j}(t)=\frac{e^{A u_{j}(t)}-1}{e^{A}-1}
\end{aligned}
$$

where $u_{j}(t)$ is the postprocessed EMG, $e_{j}(t)$ is the filtered EMG, $\alpha$ is the filtering gain coefficient, $\beta_{1}$ and $\beta_{2}$ are the recursive coefficients, $t$ is the electromechanical delay, $a_{j}(t)$ is the muscle activation, $A$ is the nonlinear shape factor and $j$ is the muscle index. MTU-specific neural activation is used in combination with muscle and tendon kinematics to solve for the dynamic equilibrium between fibers and series elastic tendons in the computation of muscle force using a Hill-type muscle model (eq. 3) [16]:

where $F^{t}$ is the tendon force, $F^{m}$ is the fiber force, $\phi\left(l^{m}\right)$ is the pennation angle, $l^{m}$ is the

$$
\begin{aligned}
& F^{m t}=F^{t}=F^{m} \cos \left(\phi\left(l^{m}\right)\right) \\
& =\left[a(t) f\left(l^{m}\right) f\left(v^{m}\right)+f_{p}\left(l^{m}\right)\right] F^{\max } \cos \left(\phi\left(l^{m}\right)\right)
\end{aligned}
$$

fiber length, $v^{m}$ is the fiber velocity, $f\left(l^{m}\right)$ is the force due to the fiber force-length relationship, $f\left(v^{m}\right)$ is the force due to the fiber force-velocity relationship, $f_{p}\left(l^{m}\right)$ is the force due to the passive force-length relationship and $a(t)$ is the muscle activation from eq. 2. Computed muscle-tendon forces are subsequently transferred onto skeletal joints via moment arms (from the musculotendon kinematics component ) to compute resulting moments about two sagittal DOFs. These included knee flexion-extension and ankle plantar flexion-dorsiflexion. Estimated joint moments are used to compute exoskeleton control command as follows (eq. 4):

$$
\tau_{c}=M_{j}\left(u_{m}(t), P_{j}\right) * A_{c t e}
$$

where $\tau_{c}$ is the exoskeleton control command, $M_{j}$ is the estimated moment for joint $\mathrm{j}$ as a function of post-processed EMG of muscles $\mathrm{m}$ acting on the joint $\mathrm{j}$ and $P_{j}$ the position of 
joint j, and $A_{\text {cte }}$ is the task's specific constant (cte) gain that determines the assistance level. The assistance level $A_{c t e}$, once chosen, remains constant throughout the experiment. Therefore, given a chosen $A_{c t e}$, there is a fixed mapping between EMG and exoskeleton assistive moment.

The model-calibration component (Fig. 1D) identifies subject-specific model parameters that vary nonlinearly across subjects' anthropometric features and force-generating capacities. These include the muscle twitch activation/deactivation time constants, EMG-to-activation nonlinearity factor, muscle optimal fiber length, tendon slack length, and muscle maximal isometric force. The initial nominal parameters are repeatedly refined as part of a leastsquares optimization procedure so that the mismatch between the EMG-driven model's predicted joint moments and those measured by the strain gauges of the robotic exoskeleton is minimized [116].

The low-level exoskeleton controller (Fig. 1E) transfers joint moment estimates to the main exoskeleton PID controller [32], which distributes moment commands to the motor drive and microcontroller (STMicroelectronics, Switzerland) of each joint.

\subsubsection{Communication Framework}

The whole real-time modelling framework (Fig. 1) operates on a portable low-power embedded system (Raspberry Pi 3, Raspberry Pi Foundation, UK) with a quad-core processing unit $(1.20 \mathrm{GHz})$ and $1 \mathrm{~GB}$ of RAM memory. A custom board was built to digitalize EMG data recorded from active sensors with built-in hardware filtering (13E200, OttoBock, Duderstadt, Germany). The custom board was further connected to a wearable computer board through a Serial Peripheral Interface (SPI) bus to enable bidirectional communication. The robotic exoskeleton was connected to the embedded system running the modelling framework via a controller area network (CAN) protocol and a CAN board (Pican2, SP Pang, UK).

\subsubsection{Experimental Procedures}

Experimental procedures were divided into two parts, conducted on two consecutive days. The first part established the personalized musculoskeletal model and exoskeleton configuration, i.e., identified subject-specific model parameters and alignment of the humanto-exoskeleton DOF center of rotation. The second part encompassed the exoskeleton voluntary control experiments reported in the Results section.

First part - musculoskeletal model and exoskeleton personalization: Motion capture data were recorded $(150 \mathrm{~Hz})$ using a seven-camera system (BTS S.p.A., Italy) and a set of 29 retroreflective markers placed on anatomic landmarks on the individual's lower extremities (bilaterally), pelvis, and trunk [14]. Data were recorded during one static anatomical pose and used in conjunction with the open-source software OpenSim [25] to scale a generic model 
of the human trunk-pelvis-lower extremity musculoskeletal geometry to match the subject's anthropometric features. The OpenSim musculoskeletal geometry model had five lowerextremity DOFs (per extremity side), including hip flexion-extension, internal-external rotation, adduction-abduction, knee flexion-extension, and ankle plantar flexiondorsiflexion. The model included 12 musculotendon units (per lower extremity, i.e., Table 1) and was taken from the literature [52]. During the scaling process, virtual markers were placed on the generic musculoskeletal geometry model based on the position of the experimental markers from the static pose. The musculoskeletal geometry model scaling procedure adjusted the anthropomorphic properties of anatomical segments (i.e., size, mass and inertial properties) as well as MTU insertions and origins and MTU-to-bone wrapping

Table 4-1:. EMG-to-MTU mapping. Muscle groups from which experimental electromyography (EMG) signals were recorded and the associated musculotendon units (MTUs) in the computational modeling framework (Fig. 1C) that were driven by these EMG signals. In this study, the gastrocnemius medialis EMG also drove the gastrocnemius lateralis MTUs. The vastus intermedius EMG activity was calculated as the mean between the vastus lateralis and vastus medialis EMG signals. The long head and short head of the biceps femoris were driven by the same EMG signal. The same applied to the semimembranosus and semitendinosus.

RECORDED MUSCLE MUSCULOTENDON UNIT (MTU) EMG

\begin{tabular}{r|c|} 
MESTROCNEMIUS & gastrocnemius lateralis and gastrocnemius \\
SIBIALIS ANTERIOR & medialis \\
\cline { 2 - 2 } SOLEUS & tibialis anterior \\
\cline { 2 - 2 } VASTUS LATERALIS & vastus intermedius, vastus lateralis \\
VASTUS MEDIALIS & vastus intermedius, vastus medialis \\
\cline { 2 - 2 } SEMIMEMBRANOSUS & semimembranosus, semitendinosus \\
\cline { 2 - 2 } BICEPS FEMORIS & biceps femoris short head, biceps femoris long \\
RECTUS FEMORIS & head \\
\cline { 2 - 2 } & rectus femoris \\
&
\end{tabular}


Section: Voluntary Control of Wearable Robotic Exoskeletons by Patients with Paresis via Neuromechanical Modeling

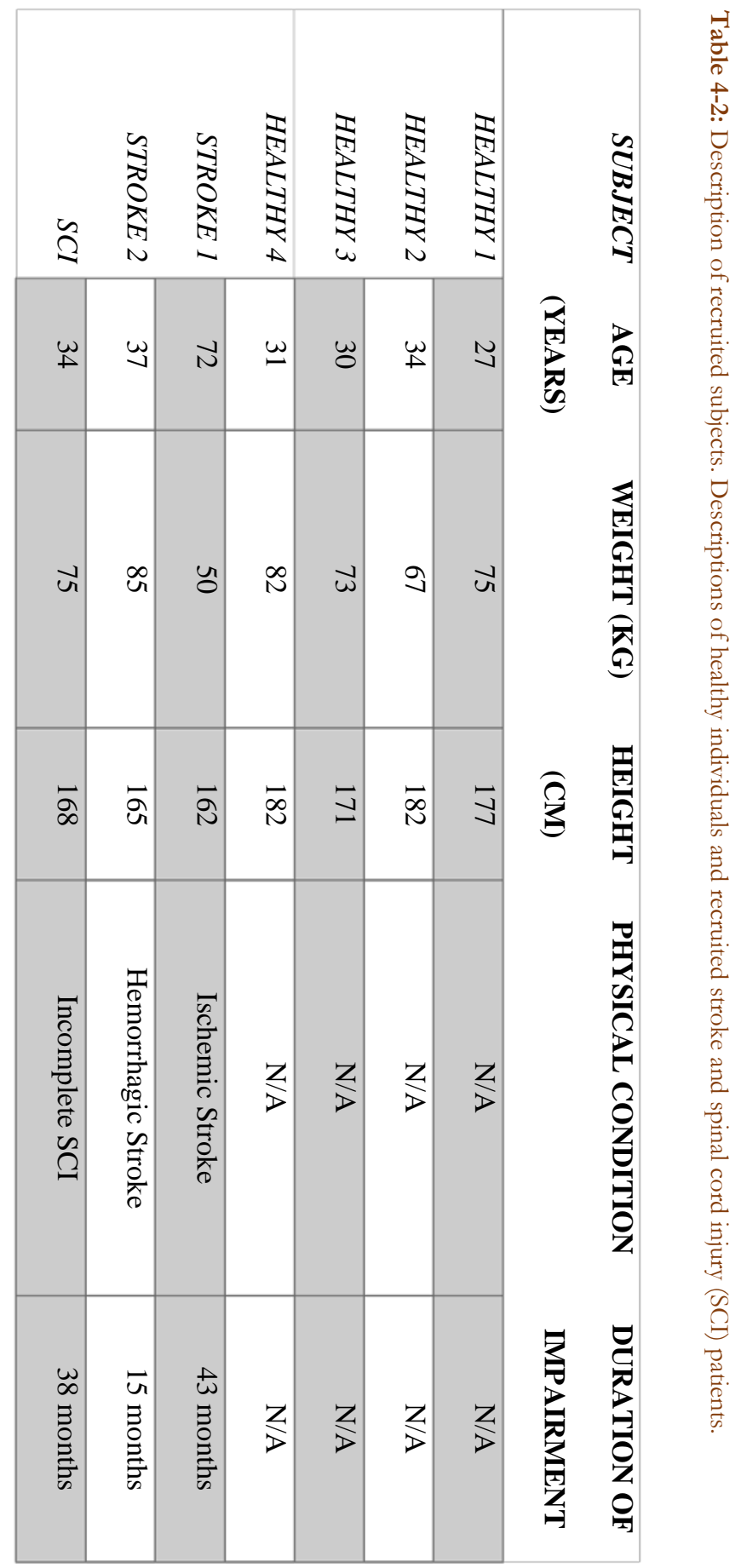


points. These properties were linearly scaled on the basis of the relative distances between the actual subjects' experimental and corresponding virtual markers [25]. Subsequently, musculotendon parameters were identified, including optimal fiber length and tendon slack length. Because these do not scale proportionally across anthropometric profiles, we employed nonlinear optimization [117].

This was used to iteratively adjust both optimal fiber length and tendon slack length to maintain the consistency of the normalized fiber length-joint angle relationship between an individual and a generic musculoskeletal model across the joint range of motion. This provided initial values for the model-calibration procedure (Fig. 1D) described in the Computational Modeling Framework section.

Subsequently, subjects wore the robotic exoskeleton for joint alignment. EMG signals were measured $(1000 \mathrm{~Hz})$ from eight thigh and shank muscles (Table 1) using dry non-disposable bipolar electrodes (13E200 MyoBock, OttoBock Health Care, GmbH, Germany). Each individual was asked to perform maximal voluntary contractions (MVCs) in isometric conditions with the exoskeleton constraining knee and ankle rotations to predefined arrangements: $45 \mathrm{deg}$ knee flexion and 0 deg ankle dorsiflexion. EMG electrodes provided on-board hardware preamplification and filtering to generate output linear envelopes. The resulting envelope peak-processed values were used for EMG normalization. EMG peak values were automatically obtained and saved to a file. The associated joint moment produced during these MVC contractions was not recorded and not used in the subsequent experiments.

After MVCs, subjects performed an additional five cycles of isometric knee flexion-extension followed by five cycles of ankle plantar flexion-dorsiflexion with each joint fixed in an angular position corresponding to the middle of its range of motion (ROM). During these tasks, the exoskeleton built-in strain gauges measured the sagittal knee and ankle joint moments exchanged between the user and the exoskeleton structure. The measured moments were used for the model-calibration step (Computational modelling framework section, Fig. 1D). The paretic patients were instructed to reach their maximal moment contraction, whereas healthy subjects were instructed to exert only a fraction of maximal moment (between \pm 25 and $\pm 40 \mathrm{Nm}$ ) due to strain gauge sensing limits (maximum range of $\pm 50 \mathrm{Nm}$ ). After calibration, the gains for different exoskeleton support levels were determined, including low-gain (LG) and high-gain (HG) support (see Table S2 in the supplementary material for the gain selected for the patients). The LG value was tuned to provide a comfortable, perceptible level of assistance. The HG value was manually tuned to achieve an increase of approximately $50 \%$ in exoskeleton moment for the same EMG level with respect to the LG condition. These gains were also empirically and individually adjusted, accounting for each subject's feedback during the outside the exoskeleton (OUT-type) conditions (see section below). 
Section: Voluntary Control of Wearable Robotic Exoskeletons by Patients with Paresis via Neuromechanical Modeling

Second part - exoskeleton voluntary control tests: The calibrated subject-specific EMG-driven model was employed in real-time to test individuals' voluntary control of the robotic exoskeleton. First, subjects were seated in a medical chair outside the robotic exoskeleton. The exoskeleton was firmly secured next to the subject via custom-made support, i.e., OUTtype tests. This enabled quantifying the influence of our proposed EMG-driven musculoskeletal model alone on both exoskeleton control accuracy and neuromuscular activity, i.e., without the physical support provided to the user's leg by the robotic exoskeleton. This was central for assessing whether robotic assistance could be determined purely from patients' voluntary neuromuscular function. Second, the subjects wore the exoskeleton, i.e., inside the exoskeleton (IN-type) condition tests. This enabled observing the behavior of the composite human-exoskeleton system, i.e., our proposed model-based HMI in conjunction with the physical support provided to the human by the robotic exoskeleton. This was used to verify whether human-exoskeleton synchronization could be achieved to enable neuromuscular effort reduction. Both OUT- and IN-type tests included single-DOF (i.e., the ankle or knee joint individually) and multi-DOF control tasks (i.e., the ankle and knee simultaneously).

During the single-DOF tasks, subjects were instructed to perform a series of joint rotations that enabled the exoskeleton joints to be moved to track a monitor-displayed reference trace. Rotations were performed first with the ankle joint and then with the knee joint. For each joint, rotations were performed first with low support gain and then with high support gain.

Table 4-3: Range of motion (in degrees) employed during experiments. Ranges of motion were personalized for each recruited patient to avoid muscle overstretching and testing outside of safe boundaries. Knee flexion and ankle plantar flexion-dorsiflexion are indicated by negative angles.

SUBJECT KNEE ANKLE KNEE ANKLE
ANGLE
ANGLE
ANGLE (IN-
ANGLE (IN-
(OUT-
(OUT-TYPE)
TYPE)
TYPE)

TYPE)

\begin{tabular}{|r|c|c|c|c|} 
HEALTHY & -80 to -40 & -10 to 10 & -80 to -40 & -10 to 10 \\
\cline { 2 - 5 } STROKE 1 & -80 to -40 & 0 to 15 & N/A & N/A \\
\cline { 2 - 5 } STROKE 2 & -80 to -40 & -10 to 10 & -80 to -40 & -10 to 10 \\
\cline { 2 - 5 } SCI & -80 to -40 & -10 to 10 & -70 to -50 & -10 to 10
\end{tabular}


This involved moving the exoskeleton knee or ankle joint to track a joint angle trajectory that spanned a predefined ROM for each joint. ROMs were specifically adjusted for the stroke and SCI patients to avoid joint overextension that would overstretch the muscles since the patients' muscles were found to be stiffer than those of healthy individuals (Table 3). Figs 2 and 3 depict the single-DOF trajectories. Each tracking trial was designed to last for 30 seconds. Each trial was repeated five times.

During the multi-DOF tasks, the reference motions to be tracked involved simultaneous knee flexion-extension and ankle plantar flexion-dorsiflexion. Table 3 reports subjectspecific ROMs selected for the participants. Subjects were presented with a graphical user interface displaying real-time information about the kinematic arrangement of the robotic exoskeleton via a stick figure depicted in blue (Fig. 4). A second stick figure, depicted in green, represented the target stick figure to be reached over time (Fig. 4 and Movie 1). The multi-DOF tests were performed first with low support gain and then with high support gain. Each trial was repeated 5 times for the healthy subjects and up to 5 times for the patients.

\subsubsection{Robotic exoskeleton}

All tests were performed using a multijointed robotic exoskeleton (H2, Technaid, Spain) equipped with six sagittal non-back-drivable motors (Maxon, Switzerland) with harmonic drive (Harmonic Drive, US), i.e., three motor-drives per leg side. The actuated DOFs were hip flexion-extension (20 deg flexion, $100 \mathrm{deg}$ extension), knee flexion-extension (100 deg flexion, 0 deg extension), and ankle plantar flexion-dorsiflexion (20 deg for both plantarflexion and dorsiflexion). The robotic exoskeleton had six strain gauges (i.e., one per joint) for measuring human-exoskeleton joint interacting moments and four footswitches for measuring foot-ground interaction. In this study, we employed a low-level PID controller for each motor that operated in the moment domain. The PID was fine-tuned for moment tracking across a range of mechanical loads [32]. The robotic exoskeleton was powered by a lithium-ion battery with five-hour autonomy.

\subsubsection{Human participants}

We recruited four healthy subjects along with three neurologically impaired patients, including one patient with SCI and two chronic hemiparetic stroke patients (Table 2). These patients were selected because they are representative of the majority of the paretic patient population [118], for whom the effectiveness of rehabilitation robotics with respect to classic rehabilitation has not been demonstrated quantitatively yet [85], [86]. Experiments were conducted on each patient's most affected side, involving voluntary rotation of two sagittal DOFs in the knee and ankle joints. The dominant leg was used for the experimental tests in healthy individuals. The SCI patient did not participate in the multi-DOF OUT-type test due to time constraints, and stroke patient 1 did not participate in the IN-type test. 


\section{Knee}
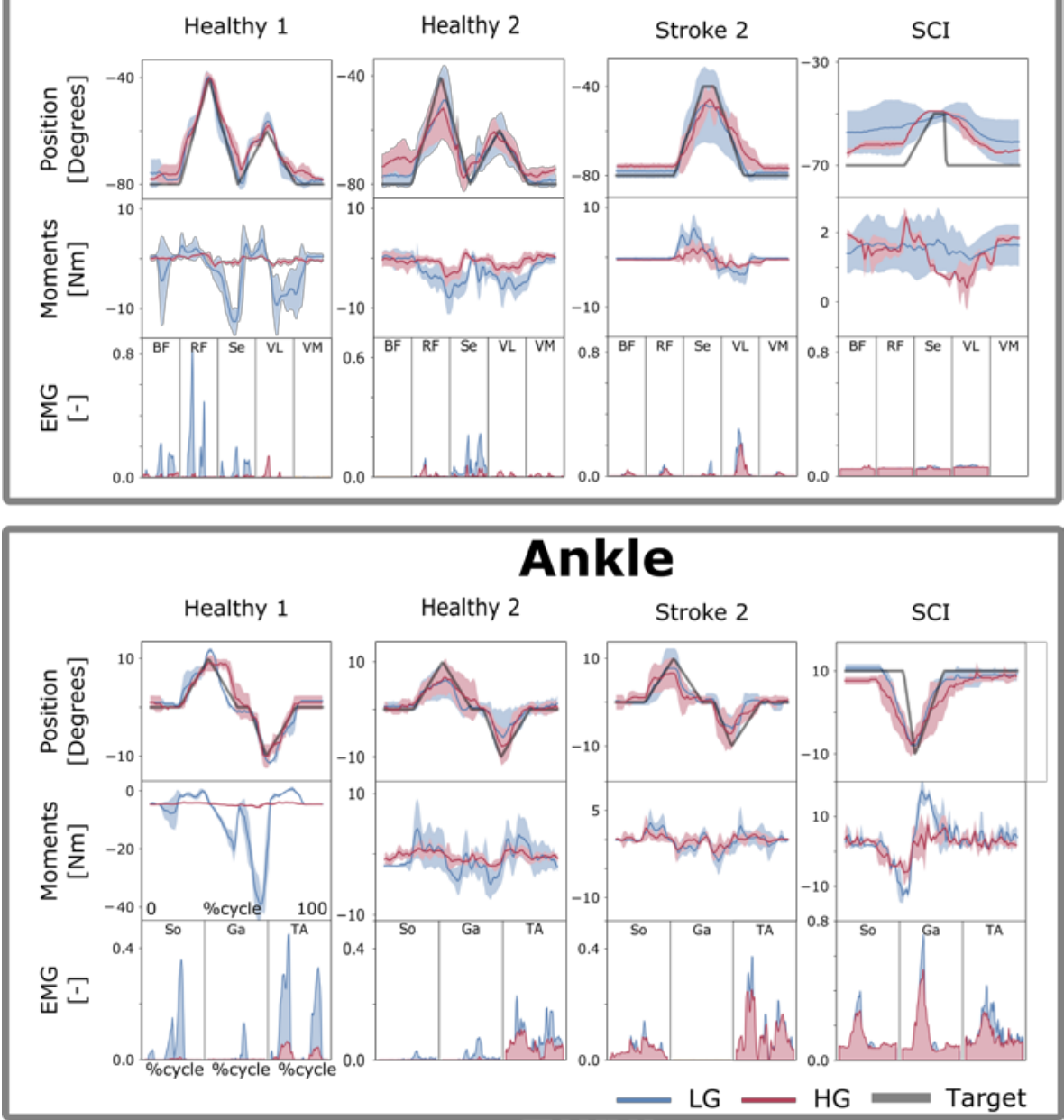

Figure 4-2: Tracking task performance during single-DOF IN-type tests. Exoskeleton joint angular position, electromyogram (EMG) data and model-based estimates of joint moments are reported during tasks with one degree of freedom (DOF). Data are reported as averages across all tracking trials with standard deviations (shaded area). They are reported for the low-gain (LG) and high-gain (HG) exoskeleton assistance levels and as a function of percent cycle, i.e., where $0 \%$ and $100 \%$, respectively, represent the beginning and the end of the tracking trajectory (Target). Target trajectories are personalized to each patient (Table 3) as detailed in the Materials and Methods section. The results are relative to tests inside the exoskeleton, i.e., IN-type tests. Data are reported for two representative healthy subjects (Healthy 1-2), two stroke patients (Stroke 1-2) and one incomplete spinal cord injury (SCI) patient. The results are reported both for the individual control of the exoskeleton ankle plantar flexion-dorsiflexion DOF and for that of the exoskeleton knee flexion-extension DOF. EMGs are relative to muscles, including the biceps femoris (BF), rectus femoris $(\mathrm{RF})$, semimembranosus $(\mathrm{S})$, vastus lateralis $(\mathrm{VL})$ and vastus medialis $(\mathrm{VM})$, soleus (So), gastrocnemius medialis $(\mathrm{Ga})$ and tibialis anterior $(\mathrm{TA})$, as shown in Table 1. 


\subsubsection{Numerical Analysis}

We quantified the model real-time performance via mean computation time and standard deviation across all simulation frames from all subjects and tasks. The 95\% confidence interval was estimated using Chebyshev's theorem, i.e., expected value $=$ mean $\pm 4.47 \cdot$ std. This could be applied with no assumptions about the normality of computation time distributions. Similarity metrics between reference trajectories and exoskeleton joint kinematic trajectories were assessed via the Pearson product-moment correlation coefficient and the root mean square error for the two considered conditions (IN-type and OUT-type).

Across all tests, data analysis was performed using Python and the NumPy library [119]. In Experiments 1 and 2 (see Results section, Figs 7, S3, S4), we verified whether our framework could induce EMG amplitude reduction while assuring that there was no loss of tracking quality. The histograms (Figs 7, S3, S4) represent the cumulative of the mean of the normalized EMG for each muscle. We used eq. 5 to quantify variability in cumulative EMG amplitude (black vertical lines on top of the bar):

where $\mathrm{W}$ is the number of repetitions, $\mathrm{N}$ is the number of EMG samples, $x_{i}$ is the EMG

$$
\sum_{\text {muscle }} \frac{\sum_{\text {repetition }}\left(\sqrt{\frac{\sum_{i=1}^{N}\left(x_{i}-\bar{x}\right)^{2}}{N-1}}\right)}{W}
$$

sample for time $\mathrm{i}$, and $\bar{x}$ is the mean of all EMG samples. In Experiment 3 (i.e., see Results section, Figs 6, S8), reduction in EMG variability was assessed via changes in standard deviation as well as in normalized standard deviation. The EMG variability measure was computed as follows:

where $W$ is the number of repetitions, $\overline{E M G}$ is the mean of the EMG sample, and $N$ is the number of EMG samples. The normalized std was computed using the following formula:

$$
\begin{aligned}
& x_{s t d}=\sqrt{\frac{\sum_{i=1}^{W}\left(\left(\sum_{m u s c l e} \overline{E M G}\right)_{i} \overline{\left(\sum_{m u s c l e} \overline{E M G}\right)}\right)^{2}}{N-1}} \\
& x_{\overline{s t d}}=\frac{x_{\text {std }}}{x_{\text {mean }}}
\end{aligned}
$$

where $x_{s t d}$ is the std computed in eq. 6 and $x_{\text {mean }}$ is the mean of the EMG signals. 
Section: Voluntary Control of Wearable Robotic Exoskeletons by Patients with Paresis via Neuromechanical Modeling

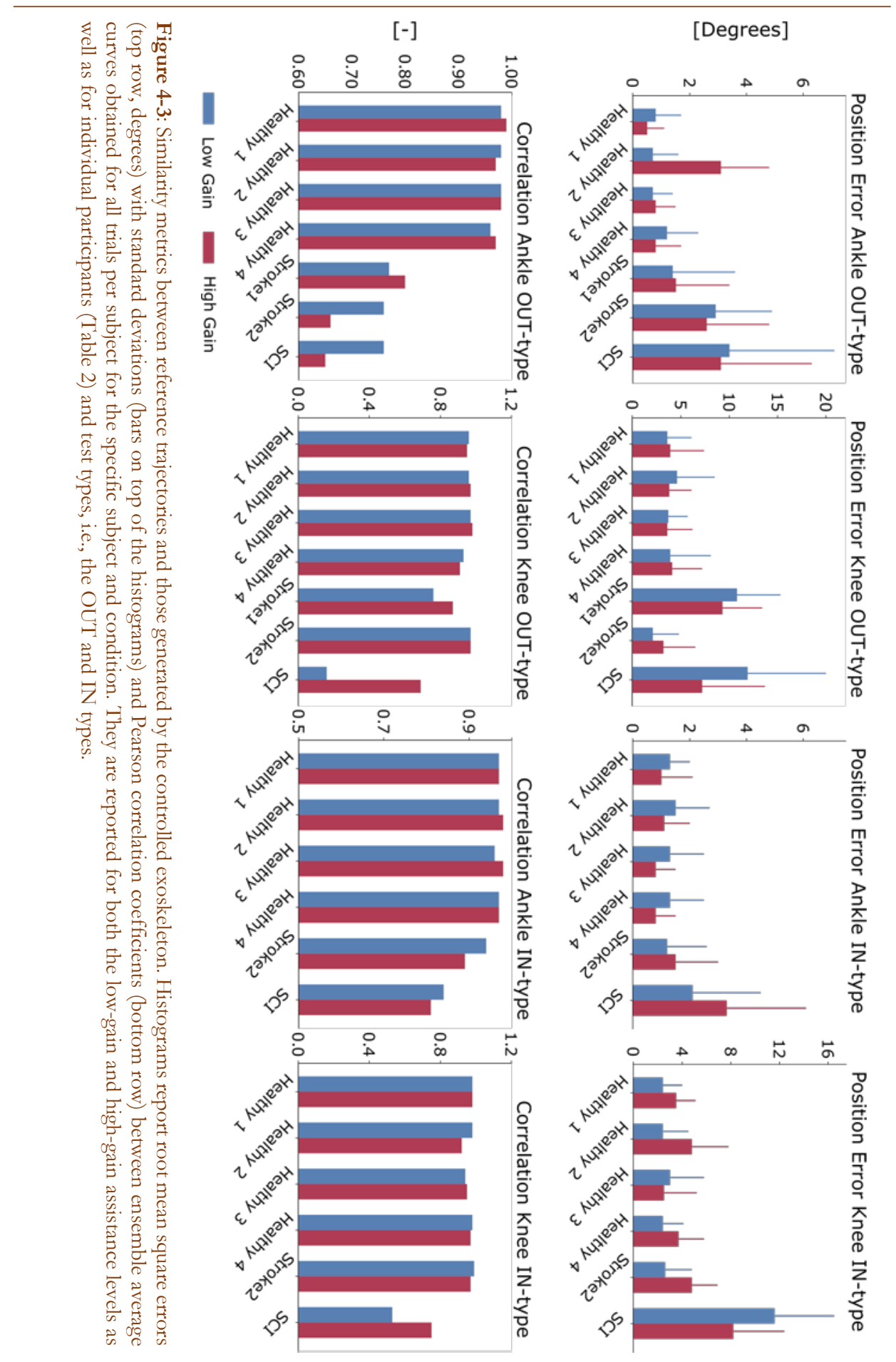




\subsection{Results}

\subsubsection{Tracking accuracy of single-DOF trajectories under different assistance levels}

The first test assessed subjects' ability to control exoskeleton ankle and knee DOFs individually to track monitor-displayed reference trajectories, i.e., Figs 2 and 5, Movie 1.

This assessed how tracking accuracy varied as a function of increasing robotic exoskeleton assistance levels, i.e., from LG to HG. During single-DOF tasks, the subjects were in full control of the robotic exoskeleton's knee and ankle DOFs.

Figs 2 and 5, respectively, show results for the OUT- and IN-type tests for both ankle plantar flexion-dorsiflexion and knee flexion-extension. Results are presented for two stroke patients, one SCI patient and two representative healthy subjects. The results from the remaining subjects are reported in supplementary Figs S1-S2. Fig. 3 shows that across all healthy subjects, test types (OUT and IN), DOFs, and gains (LG and HG), the maximal tracking errors were always $<8$ degrees with a correlation coefficient always $>0.85$. Tracking errors for the patients were on average $5.5 \pm 3.1$ degrees with correlation coefficients always $>0.6$ during IN-type tests. However, during OUT-type tests with LG, tracking errors reached higher values for the SCI patient (i.e., $13 \pm 7$ degrees) these substantially decreased when employing HG assistance levels (i.e., 6.4 6 6.1 degrees). Overall, HG assistance offered comparable or reduced tracking capacity with respect to LG assistance but was still within acceptable boundaries (Fig. 3).

Specifically, for the ankle joint during OUT-type tests (Figs 3 and 5), the mean tracking error and standard deviation (std) across healthy subjects and patients, respectively, measured $1.58 \pm 1.64$ degrees (LG) and 1.77 \pm 1.6 degrees (HG), while the correlation coefficients were $0.88 \pm 0.10$ (LG) and $0.86 \pm 0.14$ (HG). During IN-type tests (Figs 2-3), the tracking error measured $1.45 \pm 1.35$ degrees (LG) and $1.41 \pm 1.28$ degrees (HG), with correlation coefficients of $0.94 \pm 0.04$ (LG) and $0.93 \pm 0.06$ (HG). During the OUT-type tests (Figs 3 and 5), for the knee joint control, the tracking error measured 5.8 \pm 3.98 degrees (LG) and 5.01 \pm 3.62 degrees (HG), with correlation coefficients of $0.81 \pm 0.27$ (LG) and $0.90 \pm 0.09$ (HG). During IN-type tests (Figs 2-3), the tracking errors measured 4.06 \pm 2.55 degrees (LG) and 4.58 \pm 2.61 degrees (HG), with correlation coefficients of $0.90 \pm 0.16$ (LG) and $0.92 \pm 0.07$ (HG).

\subsubsection{Modulation of neuromuscular activity}

The second test quantified the effect of our proposed model-based HMI on the modulation of neuromuscular activity. This was evaluated by examining modulations in normalized EMG and resulting mechanical joint moment amplitudes. Across all experiments, both EMG and resulting moments displayed the largest reduction during the IN-type tests for both healthy participants and patients; see Figs 4 and 7. 

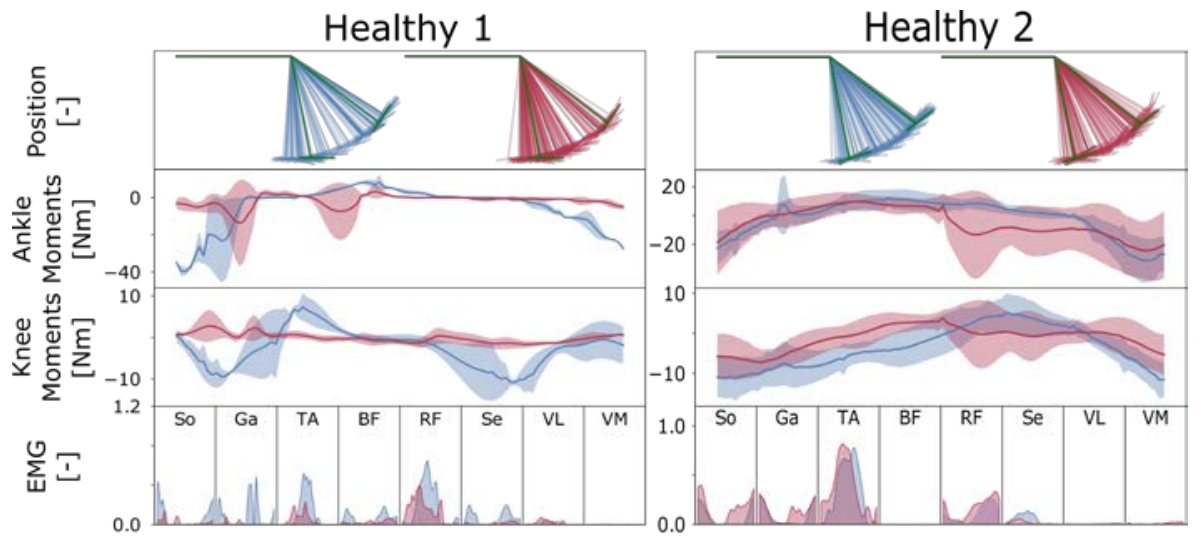

Stroke 2
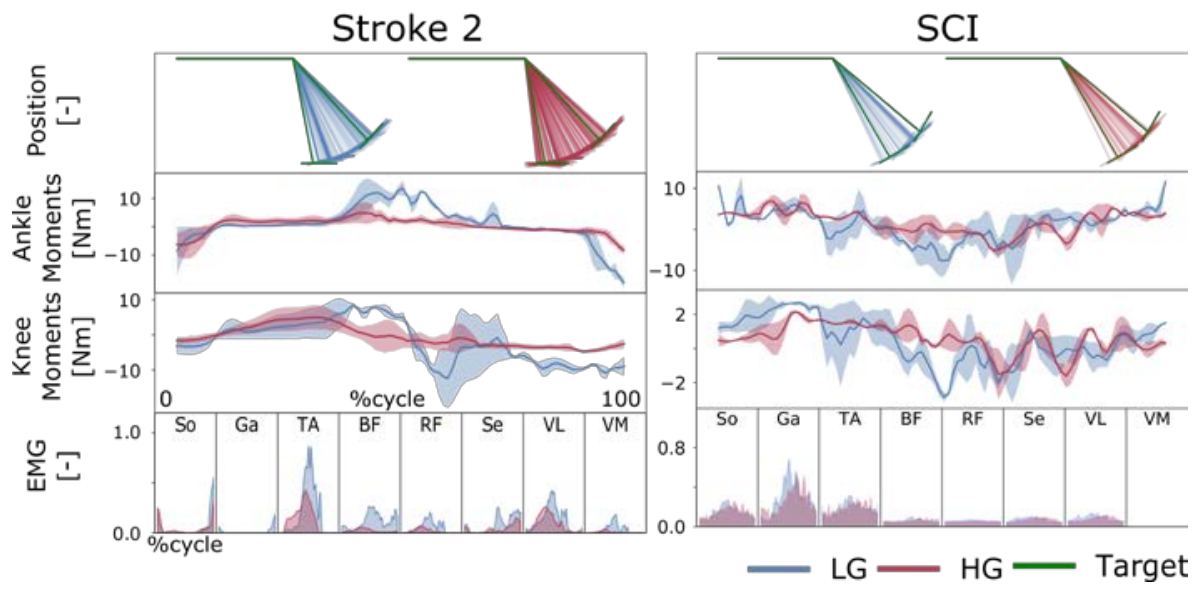

Figure 4-4: Tracking task performance during multi-DOF IN-type tests. Exoskeleton knee and ankle joint angular positions are reported by means of a stick figure. The green figure represents the target multiple-joint position to be attained. The blue and red stick figures represent the subject's voluntarily controlled exoskeleton trajectory obtained using low-gain (LG) and high-gain (HG) assistance levels, respectively. The target positions were changed automatically when the user was within \pm 5 degrees of the target position. Model-based estimates of joint moments are reported about both the knee flexion-extension and ankle plantar flexion-dorsiflexion degrees of freedom (DOFs). Data are reported as averages across all tracking trials with standard deviations (shaded area). They are reported as a function of percent cycle, i.e., where $0 \%$ and $100 \%$, respectively, represent the beginning and the end of the tracking trajectory (Target). The results are relative to tests inside of the exoskeleton, i.e., IN-type tests. Data are reported for two representative healthy subjects (Healthy 1-2), one stroke patient (Stroke 2) and one incomplete spinal cord injury (SCI) patient, as shown in Table 2. Recorded electromyograms (EMGs) are relative to muscles including the biceps femoris $(\mathrm{BF})$, rectus femoris $(\mathrm{RF})$, semimembranosus $(\mathrm{S})$, vastus lateralis (VL) and vastus medialis (VM), soleus (So), gastrocnemius medialis (Ga) and tibialis anterior (TA), as presented in Table 1. 

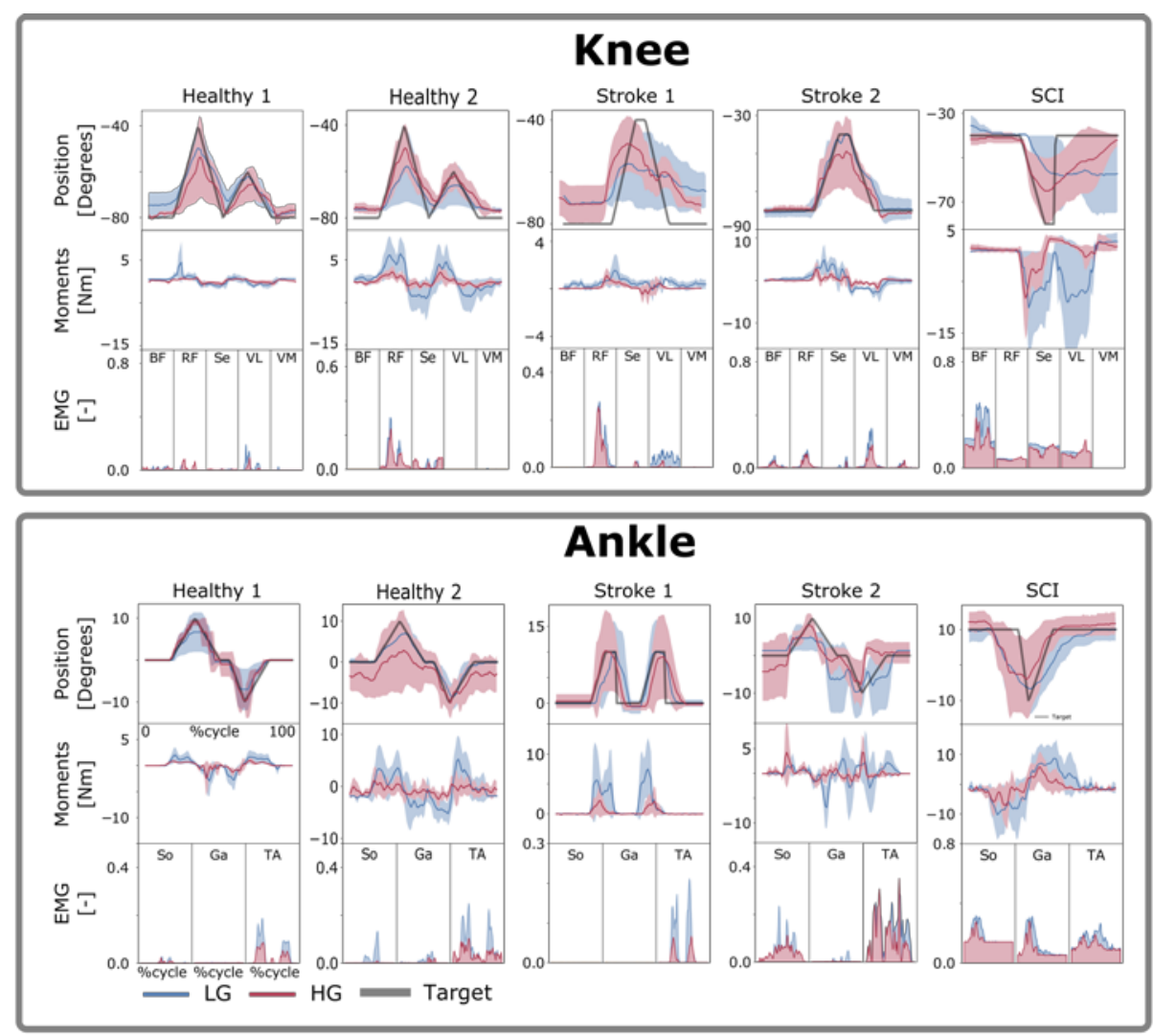

Figure 4-5: Tracking task performance during single-DOF OUT-type tests. Exoskeleton joint angular position, electromyogram (EMG) data and model-based estimates of joint moments are reported during tasks with one degree of freedom (DOF). Data are reported as averages across all tracking trials with standard deviations (shaded area). They are reported for the low-gain (LG) and high-gain (HG) exoskeleton assistance levels and as a function of percent cycle, i.e., where $0 \%$ and $100 \%$, respectively, represent the beginning and the end of the tracking trajectory (Target). Target trajectories are personalized to each patient (Table 3) as detailed in the Materials and Methods section. The results are relative to tests outside of the exoskeleton, i.e., OUT-type. Data are reported for two representative healthy subjects (Healthy 1-2), two stroke patients (Stroke 1-2) and one incomplete spinal cord injury $(\mathrm{SCI})$ patient. The results are reported both for the individual control of the exoskeleton ankle plantar flexion-dorsiflexion DOF and for that of the exoskeleton knee flexion-extension DOF. EMGs are relative to muscles including the biceps femoris $(\mathrm{BF})$, rectus femoris $(\mathrm{RF})$, semimembranosus (S), vastus lateralis $(\mathrm{VL})$ and vastus medialis (VM), soleus (So), gastrocnemius medialis (Ga) and tibialis anterior (TA), as shown in Table 1. The EMG for the SCI patient has a large offset due to the high amplification needed. 
Single-DOF experiments: For the ankle joint during OUT-type tests (Fig. 5), the cumulative EMG amplitude (i.e., the sum of the mean EMG) decreased for all healthy subjects and patients from LG to HG. The cumulative EMG decreased from $0.04 \pm 0.03$ to $0.02 \pm 0.001$ for healthy subject 1 , from $0.08 \pm 0.04$ to $0.04 \pm 0.01$ for healthy subject 2 , from $0.12 \pm 0.06$ to $0.08 \pm 0.04$ for healthy subject 3 and from $0.07 \pm 0.008$ to $0.04 \pm 0.004$ for healthy subject 4 . The cumulative EMG decreased from $0.03 \pm 0.02$ to $0.01 \pm 0.008$ for stroke patient 1 , from $0.16 \pm 0.03$ to $0.13 \pm 0.08$ for stroke patient 2 and from $0.43 \pm 0.07$ to $0.35 \pm 0.04$ for the SCI patient.

For the ankle joint during IN-type tests (Figs 2, S3), cumulative EMG amplitude decreased from LG to HG, for all healthy subjects and patients, i.e., healthy subject 1 (from $0.16 \pm 0.01$ to $0.02 \pm 0.001$ ), healthy subject 2 (from $0.09 \pm 0.04$ to $0.06 \pm 0.005$ ), healthy subject 3 (from $0.14 \pm 0.02$ to $0.07 \pm 0.06$ ) and healthy subject 4 (i.e., from $0.04 \pm 0.03$ to $0.01 \pm 0.009$ ). For stroke patient 2 , we observed a small increase from $0.12 \pm 0.06$ to $0.13 \pm 0.07$, whereas the SCI patient showed a small reduction from $0.40 \pm 0.03$ to $0.39 \pm 0.007$.

For the knee during OUT-type tests (Fig. 5), we observed decreases or steady values for all healthy subjects and patients. This corresponded to a change in EMG amplitude from $0.04 \pm 0.006$ to $0.03 \pm 0.01$ for healthy subject 1 , from $0.30 \pm 0.03$ to $0.12 \pm 0.06$ for healthy subject 3 and from $0.02 \pm 0.02$ to $0.04 \pm 0.02$ for healthy subject 4 . For healthy subject 2 , we observed an unaltered signal level (i.e., a reduction of 0.001). For users with paresis, we observed reductions from $0.11 \pm 0.06$ to $0.07 \pm 0.02$ for stroke patient 1 , from $0.11 \pm 0.01$ to $0.08 \pm 0.04$ for stroke patient 2 and from $0.65 \pm 0.05$ to $0.52 \pm 0.06$ for the SCI patient. For the knee in the IN-type tests (Figs 2, S4), we observed reductions in EMG amplitude for all healthy subjects and patients. Specifically, the amplitude changed from $0.21 \pm 0.02$ to $0.04 \pm 0.003$ for healthy subject 1 , from $0.07 \pm 0.01$ to $0.03 \pm 0.01$ for healthy subject 2 , from $0.23 \pm 0.04$ to $0.12 \pm 0.003$ for healthy subject 3 and from $0.05 \pm 0.01$ to $0.02 \pm 0.01$ for healthy subject 4. Among the patients with paresis, we observed a reduction from $0.09 \pm 0.3$ to $0.07 \pm 0.01$ for stroke patient 2 and $0.215 \pm 0.01$ to $0.209 \pm 0.001$ for the SCI patient.

Model-based estimates of joint moments were always modulated in response to EMG activity, as shown in Figs 2, 5, S1, and S2. Both knee and ankle joint moments displayed decreases in their mean values from the LG to the HG assistance level. This was observed for both healthy subjects and patients, with the largest reductions observed during the INtype tests. Supplementary Table S1 provides detailed quantitative values. 
Multi-DOF experiments: This testing condition assessed whether EMG and joint moment amplitude reduction could be observed in tasks relying on larger muscle sets and control of multiple joints. Reference motions to be tracked involved simultaneous knee flexionextension and ankle plantar flexion-dorsiflexion, as shown in Movie 2.

All subjects and patients were able to control the multi-DOF robotic exoskeleton and match the target positions during both OUT-type (supplementary Fig. S5-S7) and IN-type tests (Fig. 4). Fig. 7 shows that the robotic exoskeleton assistance resulted in a consistent decrease in cumulative EMG amplitude across all subjects. During OUT-type tests (supplementary Figs S5-S7), cumulative EMG amplitude decreased for all healthy subjects and patients between LG and HG. The EMG amplitude decreased for healthy subject 1 (from $0.21 \pm 0.02$ to $0.12 \pm 0.02$ ), subject 2 (from $0.64 \pm 0.16$ to $0.46 \pm 0.27$ ), subject $3(0.91 \pm 0.1$ to $0.70 \pm 0.23$ ) and subject 4 (from $0.24 \pm 0.05$ to $0.16 \pm 0.02$ ). Among patients, the cumulative EMG

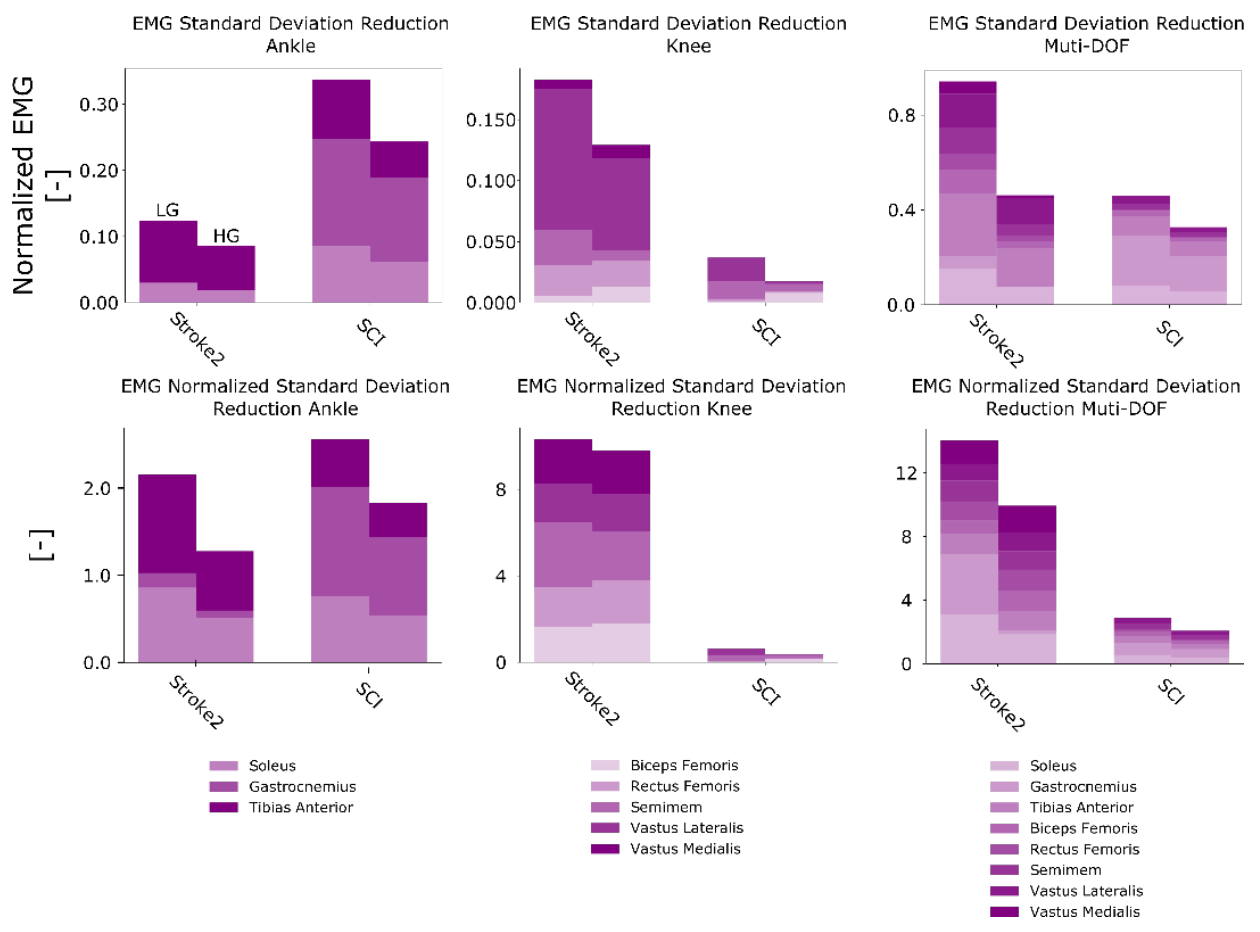

Figure 4-6: Standard deviation of mean EMG amplitude during single-DOF and multi-DOF INtype tests. Histograms report the nonnormalized standard deviation (top row) and normalized standard deviation (bottom row, eq. 7) extracted from electromyogram (EMG) data across all trials performed during single-ankle control tasks, single-knee control tasks and simultaneous ankle-knee control tasks. Histograms are reported relative to low-gain (LG) and high-gain (HG) assistance levels. Data are relative to stroke patient 2 and to the incomplete spinal cord injury patient (SCI), Table 2. 
amplitude decreased for stroke patient 1 (from $0.20 \pm 0.03$ to $0.19 \pm 0.03$ ) and stroke patient 2 (from $0.35 \pm 0.10$ to $0.27 \pm 0.05$ ). Quantitative data are not available for the SCI patient, who did not perform this test.

During IN-type tests (Figs 4 and 7, S5, S6), increased exoskeleton assistance resulted in EMG reduction for most healthy subjects and all patients, i.e., $0.61 \pm 0.003 / 0.27 \pm 0.06$ (LG/HG) for healthy subject $1,0.64 \pm 0.19 / 0.84 \pm 0.2$ for healthy subject $2,0.62 \pm 0.03 / 0.25 \pm 0.03$ for healthy subject $3,0.21 \pm 0.04 / 0.14 \pm 0.03$ for healthy subject $4,0.69 \pm 0.14 / 0.36 \pm 0.14$ for stroke patient 2 and $0.90 \pm 0.18 / 0.89 \pm 0.005$ for the SCI patient. Quantitative data are not available for stroke patient 1 who did not perform this test.

Model-based estimates of joint moments were always modulated in response to EMG activity, with knee and ankle joint moments displaying decreases in their mean values from the LG to the HG assistance levels. This was reflected in both healthy subjects and patients, with the largest reductions observed during IN-type tests (Supplementary Table S1).

\subsubsection{Variability of neuromuscular activity}

The third test assessed the extent of variability in EMG amplitude across exoskeleton assistance levels and tasks, as described by eq. 6. An index of normalized variability was also computed (eq. 7) to enable comparison between the LG and HG assistance levels while controlling for mean EMG amplitude. The results showed that increased assistance levels resulted in reduced EMG variability across all patients (Figs 6, S8), which may have practical consequences for neurologically impaired patients who are affected by spastic (and thus highly variable) EMG activity.

During OUT-type tests (Fig. S8), EMG variability across all trials (i.e., both single- and multiDOF) decreased for stroke patient 1 (from 0.48 to 0.38 ) and stroke patient 2 (from 0.82 to 0.57). After normalization, the variability decreased for stroke patient 2 (from 22.8 to 22.2) and increased for stroke patient 1 (15.2 to 17.2).

During the IN-type tests (Fig. 6), the EMG variability across all trials (i.e., both single- and multi-DOF) decreased for stroke patient 2 (1.24 to 0.67) and for the SCI patient (from 0.83 to 0.58 ), as shown in the top row histograms in Fig. 6. Similarly, after normalization, the variability decreased for stroke patient 2 (from 26 to 21) and for the SCI patient (from 6 to 4.2), as shown in the bottom row of histograms in Fig. 6. 


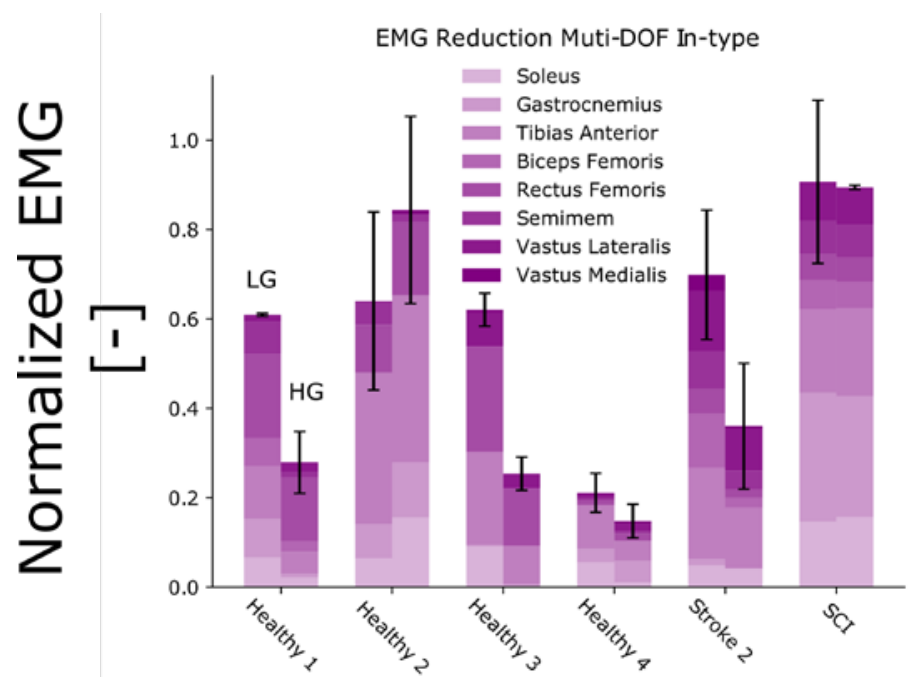

Figure 4-7: EMG amplitude modulation between low and high levels of exoskeleton assistance during multi-DOF IN-type experiments. The results are reported for tracking tasks with multiple degrees of freedom (DOFs), involving simultaneous knee and ankle joint movement. Subjects performed the experiments while wearing the robotic exoskeleton, i.e., IN-type tests. For each healthy subject (Healthy 1-4) as well as for stroke patient 2 (Stroke) and the spinal cord injury (SCI) patient (Table 2), the vertical bars report the mean normalized EMG amplitude stacked vertically for each muscle along with the standard deviation (see black vertical lines).

\subsubsection{Computational Time}

Across all subjects and tests, the proposed framework generated exoskeleton control commands with an average computational time of $7 \pm 3.7 \mathrm{~ms}-$ specifically, $5.6 \pm 3.3 \mathrm{~ms}$ for the EMG-driven modelling and $1.3 \pm 0.4 \mathrm{~ms}$ for the moment arm and tendon-muscle length. In this study, $95 \%$ of the control commands produced in a single time frame were generated within $14 \mathrm{~ms}$. This is well below the length of the muscle electromechanical delay, i.e., $\sim 30$ $80 \mathrm{~ms}$ [120], as well as the human perceivable delay in motor execution, i.e., 250 ms [121], [122].

\subsection{Discussion}

We developed and tested a model-based HMI for the voluntary control of a wearable robotic exoskeleton. We validated it in one wheelchair-bound SCI patient, two hemiparetic chronic stroke patients with residual walking capabilities and four additional healthy individuals. To the best of our knowledge, this study provides the first HMI that enables neurologically impaired patients to voluntarily control multiple DOFs in robotic exoskeletons while inducing a positive modulation of neuromuscular activity, i.e., reduction of both neuromuscular amplitude and variability. 
Assessing the benefit of robotic devices for enhancing neurologically impaired patients' movement is challenging. In this study, we focused on quantifying our model-based HMI ability to modulate EMG amplitude and variability as a function of assistive support levels. The focus was to amplify human mechanical function in a clinically viable way. This was realized by enabling the generation of (pre-defined) mechanical joint moments using reduced EMG activity via active exoskeleton support.

We established a numerical model of the human musculoskeletal system that could be scaled and calibrated to match an individual's anatomy. In this context, experimentally recorded EMG signals represented a surrogate of the neural drive to muscles. That is, EMG linear envelopes amplitude and shape features reflected the patient's disrupted motor control. Moreover, the proposed patient-specific muscle model allowed capturing the patient's impaired muscular force-generating capacity. Unlike state-of-the-art HMIs, our proposed approach allowed a robotic exoskeleton to be controlled proportionally to an individual patient's residual muscle force-generating capacity, as shown in Figs 2-3 and 6-7. Furthermore, our method provided an enhanced level of patient specificity with respect to state-of-the-art exoskeleton HMIs.

Results showed that all subjects were able to voluntarily control the exoskeleton accurately over a range of motor tasks involving rotations about single DOFs as well as multiple DOFs concurrently, i.e., Figs 2-4, 7. Subjects could control the exoskeleton to accurately track reference trajectories even when they were not directly wearing the exoskeleton, i.e., during OUT-type tests. This was hypothesized to be a challenging condition due to reduced perception of exoskeleton movement. Overall, results highlighted that patients were always in voluntary control of the exoskeleton motion, thus not being passively driven.

The results showed that an increasing level of assistance induced a decrease in the net cumulative EMG amplitude and resulting mechanical moments required to perform a motor task (Figs 2-5 and 7). Reduction was observed across all measured muscles for all subjects both during single-DOF and multi-DOF tasks. However, in a few cases, HG assistance induced higher EMG activity than LG assistance, as shown in Fig. 7, subject 2. Systematic analyses are planned as part of future work to identify direct causes. Importantly, the increased level of assistance did not degrade the user's accuracy in the reference position tracking tasks (Fig. 3).

The exoskeleton generated a support moment that was equal to a fraction of the user's net joint moment (calculated by the EMG-driven model). For higher gains the exoskeleton support moment reflected a larger fraction of the user's net joint moment. Importantly, across support gains, the reference mechanical task to be performed by the patient and exoskeleton system was always the same. That is, the underlying total net moment to be 
generated by the patient and exoskeleton system was similar across support gains. Therefore, for increasing support gains, the proportion of human-generated moment decreased while the proportion of exoskeleton-generated moment increased. This was directly reflected in our results (Figs. 2 and 4-5).

Across all subjects, the net EMG amplitude reduction was consistently associated with a concurrent reduction in EMG variability. Motor tasks performed with high assistance levels corresponded to more repeatable EMG patterns than tasks performed with low assistance levels (Fig. 6). This could be achieved only if the exoskeleton joint actuation was precisely synchronized with the patient's muscle contraction. Lower levels of human-exoskeleton synchronization would lead to the exoskeleton counteracting the patient's movements or providing suboptimal assistive moments, thereby inducing an increase in EMG magnitude and variability. Personalized models played an important role in achieving these results. Supplementary Fig. S9 shows how noncalibrated models display large discrepancies with respect to reference moments, which would hamper the controllability of exoskeletons. Patients with neurological lesions naturally present greater movement variability than healthy individuals [123], especially because of involuntary (spastic-like) muscle activity. In this context, spasticity or hyperactive stretch reflexes would be directly captured via EMGs. Because our proposed musculoskeletal model was driven by EMGs, this enabled capturing muscle force controlled by abnormal spinal neuron activity. Our results showed that our proposed model-based amplification of patient's neuromuscular function enabled tracking smooth join position trajectories despite the underlying patients' EMGs may underlie spastic activity. This may be due to the fact that the presence of high-energy spikes in the EMG (i.e. due to spastic-like EMGs) may be attenuated by the inherent visco-elasticity of the Hill-type muscle model as well as muscle-tendon small moment arms. This has the benefit of generating smooth muscle force-dependent joint moment profiles even in the presence of spikes in the input EMGs. Future work will systematically assess the ability of musculoskeletal models in attenuating EMG abnormal spiking activity and also assess its robustness with respect to abnormal activation, such as spasticity, in a rehabilitation scenario. Furthermore, future studies will assess the possibility of using high-density EMG recording to decompose the signal at the level of constituent motor unit discharges. This would enable separating physiological motor units from those displaying abnormal behavior (i.e. spasticlike). For each muscle, filtered physiological motor units only could be used to compute exoskeleton assistive moments [71].

The ability to enforce EMG pattern repeatability (i.e., reduction of EMG variability) via wearable robotic technologies is central for retraining coordinated neuromuscular control and inducing positive neuroplasticity, i.e., by preventing involuntary uncoordinated muscle activations [88]. The ability of the proposed HMI to modulate neuromuscular activity in a controlled way may be beneficial in the future for high-intensity sensorimotor training, i.e., enabling patients to perform intensive motor tasks progressively across longer periods of 
time (EMG reduction). Intensive training has been shown to improve both muscle strength and overall motor control in stroke patients [124].

Our HMI always computed exoskeleton control commands within the muscle electrophysiological delay, i.e., < $15 \mathrm{~ms}$. This computational speed was achieved using a lowpower, small-sized, and fully wearable processing unit, i.e., Raspberry Pi 3 (Raspberry Pi Foundation, UK). This enabled the subject's movement to be predicted shortly before the actual movement took place, which is important for synchronizing the exoskeleton response to the user's neuromuscular function. Moreover, this was crucial especially for supporting neurologically impaired patients who had severely reduced motor abilities but still had detectable EMG activity. This is an important advantage with respect to HMIs that actuate the wearable robot solely on the basis of the detection of externally measurable forces (i.e., external joint moments or limb-orthosis interaction force); such systems cannot provide support unless the patient is able to produce detectable muscle force or movement [76]. In the context of our experiment, this would have severely challenged both voluntary exoskeleton control and EMG reduction in the SCI and stroke patients, whose muscle strength and EMG amplitude were substantially compromised.

This study involved voluntary control of robotic knee and ankle rotations from a seated position. These exercises were selected for two reasons. First, they provided a controlled environment for testing our proposed control method for the first time in neurologically impaired individuals. Second, they mimicked physiotherapy tasks employed during earlystage rehabilitation. Two major functional impairments related to stroke are the loss of selective joint control and muscle weakness. From a clinical perspective, both issues must be addressed via safe, comfortable and feasible positions for the patient [125], [126], something that could be provided by our proposed framework. Future work will pair wearable exoskeletons with our model-based human-machine interface to track and support the patient across all recovery stages: from sitting to walking in the hospital to finally walking outside the hospital [127]. An advantage of our framework over conventional inverse dynamics is that, once calibrated, it does not need ground reaction forces, i.e., it operates as a function of EMG and joint position, which are measurable via wearable sensors. This is central for wearable robotics applications.

To the best of our knowledge, there is currently no robotic exoskeleton on the market, either in the rehabilitation domain (i.e., Lokomat) or in the assistive domains (i.e., Rewalk, HAL), that operates as a function of a patient's residual muscle force-generating capacity. This may underpin a central element hampering the ability of current robotic exoskeletons to impact neurorehabilitation. Movie 2 shows the possibility for the SCI patient to control multiple DOFs despite minimal residual motor capacity, which is key for promoting recovery even in severely affected individuals. This study was not intended to quantify direct rehabilitation 
outcome, as this requires systematic analysis on a larger patient population, which is subject of future work.

Future work will extend our proposed methodology towards more functional tasks, such as ground- and inclined-level walking, as well as stair ascending and descending. We will investigate the effect of the exoskeleton on the patient with and without the device, as well as the effect of ground reaction force on the exoskeleton combined with our framework. A limitation of this study is that we did not test how noncalibrated models would affect exoskeleton control. Longitudinal tests with different types of models (i.e., calibrated and noncalibrated) will be performed as part of our future work. The overall quality of the optimized parameters of our muscle model (maximal isometric muscle force, optimal fiber length and tendon slack length) should also be validated against in-vivo experimental values in future work. However, it was first necessary to assess whether neurologically impaired patients could achieve voluntary control of robotics knee and ankle rotation.

\subsection{Conclusion}

This study established a new patient-specific model-based HMI that can aid clinicians and physiotherapists in the assessment of patients' motor capacity and progress over time. It can enable exoskeletons to operate symbiotically to the human body by dynamically adapting to the patient's motor capacity across different stages of recovery. This will open new avenues for establishing personalized neurorehabilitation technologies where wearable robots physically interact with the patient to maximize the recovery of compromised neuromuscular targets. 


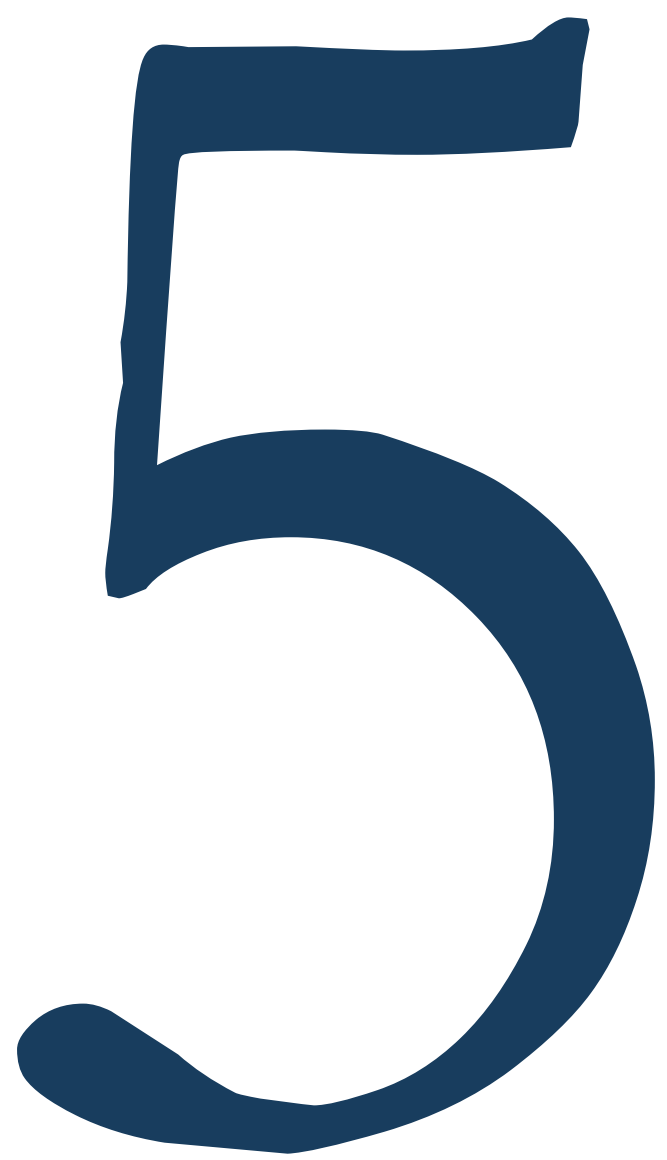




\title{
VOLUNTARY AND \\ CONTINUOUS CONTROL OF \\ ROBOTIC EXOSKELETONS \\ DURING A BROAD \\ REPERTOIRE OF \\ LOCOMOTION CONDITIONS
}

\section{Guillaume Durandau, Wolfgang Rampeltshammer, Herman van der Kooij, Massimo Sartori}

\begin{abstract}
To enable the broad adoption of wearable robotic exoskeletons in both medical and industrial settings, it is central they can effectively support a large repertoire of motor tasks. Current human-machine interfaces for exoskeletons largely rely on joint torque or kinematics patterns that are pre-computed, thereby limiting the range of supported movements. Here, we propose a human-machine interface that generates exoskeleton control commands as a direct function of an individual's neuromuscular activity, with no need to rely on pre-defined torque profiles or state machines. This is based on the use of 1) a personalized musculoskeletal model driven by electromyograms that estimate biological muscle force and joint torques and 2) an efficient torque controller based on a disturbance observer that translates biological torque estimates into exoskeleton control commands. In this study, we demonstrate the ability of the proposed approach via experiments during which three individuals voluntarily control a bi-lateral robotic ankle exoskeleton to walk across a range of gaits, i.e. two walking speeds, three ground elevations as well as the transitions across these. Across all walking conditions, the exoskeleton support enabled a reduction in electromyograms $(6 \%-15 \%)$ as well as in biological joint torques $(18 \%-25 \%)$ when compared against walking with no assistance, i.e. exoskeleton controlled in minimal impedance mode. Reduction of EMG and joint torque were also observed during the transition between tasks (16\% - 23\%). For all subjects, the total human-machine joint torque, computed as the sum of biological torque and exoskeleton torque, was preserved throughout the entire experiment. However, the exoskeleton torque proportion systematically increased while the human joint torque proportion decreased during the assisted condition when compared to the non-assisted condition, thereby making walking more economical for the user.
\end{abstract}

Keywords-locomotion, EMG, exoskeleton, lower-limb, neuromusculoskeletal modelling. 
Section: Voluntary and Continuous Control of Robotic Exoskeletons during a broad Repertoire of Locomotion Conditions

Publication-In preparation for IEEE Transaction in Robotics 


\subsection{Introduction}

Human-machine interfaces (HMIs) are central for connecting exoskeletons to the human's neuromusculoskeletal (NMS) system. Common tasks such as locomotion require complex interplay between neurons, muscles and skeletal segments. Adding an artificial mechanical system in parallel to an already complex biological system makes human locomotion dynamic assistance an open challenge.

Wearable robotic exoskeletons have great potentials for enhancing human mobility, i.e. from improving neurorehabilitation training [128] to preventing musculoskeletal injuries in factory settings [129]. For this, wearable exoskeletons need to operate in concert with the NMS system and aid a broad repertoire of movements in unstructured environments, i.e. dynamically accounting for changes in locomotion speed, direction, ground elevation as well as transitions across these.

Current HMIs do not enable humans to voluntarily control exoskeletons but operate within a priori defined conditions, i.e. they rely on pre-generated torque or angle profiles prescribed to the exoskeleton at pre-determined gait phases [29], [30], [130]-[133]. These profiles can be further optimized via human-in-the-loop methods for reducing metabolic cost [30], [132] or electromyograms (EMG) [134]. State machines are often used for switching across locomotion modes (i.e. speeds, ground elevation, tasks) [133], [135], but cannot provide continuous support across transitions, being prone to misclassification and to inadequate assistance during unknown tasks. This all limits exoskeleton applicability outside of the lab.

Proportional myoelectric controllers [107], [136] have been proposed to aid the user continuously, i.e. not only during pre-defined states. However, these have not shown conclusive results in terms of movement augmentation [137]. Joint angle proportional controller [138] based on the difference between left and right hip joint angle has shown promising experimental results for level ground locomotion and offers the possibility to assist ramp and stairs ascent and descent (metabolic results for these tasks were not shown in [138]). However, this method offers little flexibility as it based on a two-legged inverted pendulum only representing the hip joint limiting assistance to this joint.

In this paper, we propose a new HMI that enables humans to voluntarily and continuously control a bi-lateral robotic ankle exoskeleton across a broad range of locomotion conditions as well as transitions across conditions, with no assumptions on the expected movement modes. The proposed HMI does not rely on a priori defined torque profiles. The HMI highlevel controller is based on a person-specific EMG-driven musculoskeletal model that computes exoskeleton reference torque profiles based on an individual's neuromuscular function estimates.

We first describe the structure of our proposed HMI as well as that of the bilateral ankle exoskeleton employed in this study. Then, we present the experimental procedures for 


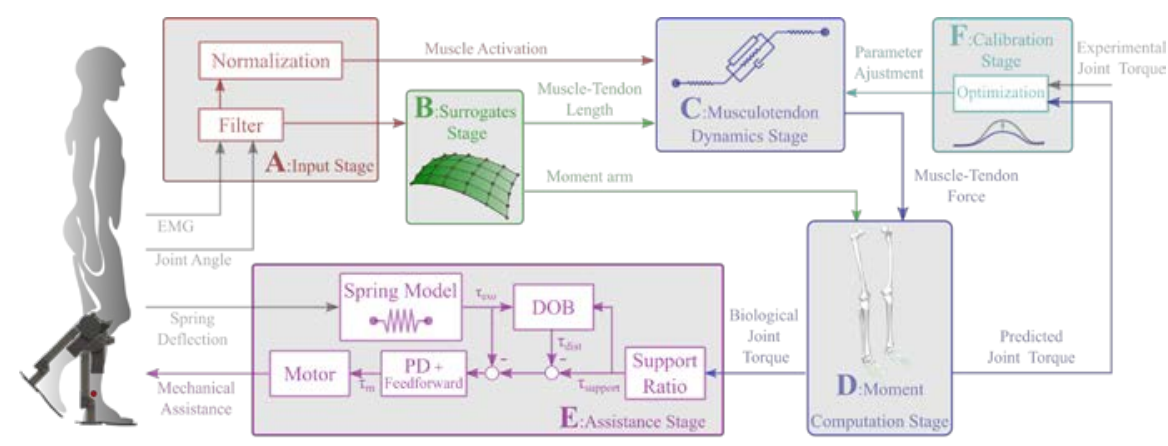

Figure 5-1: Schematic representation of our HMI.

testing the efficacy of our proposed approach as well as the quantitative analyses and results emerging from our experiments. Finally, we discuss our study implications, limitations and future work.

\subsection{Methods}

Fig. 5-1 represents our proposed HMI scheme. Each constituent block is detailed in the remainder of this section.

\subsubsection{High-level control via EMG-driven musculoskeletal modelling}

To assure voluntary and continuous control, exoskeleton commands are computed as a direct function of the subject's estimated joint torque. EMGs were recorded, amplified and filtered directly by the surface electrodes using proprietary signal detection and acquisition system (AxonMaster 13E500, Ottobock, Germany) (Fig. 5-1-A, input Stage). Filtered EMGs are normalized using pre-recorded maximal voluntary contractions to compute muscle excitation. The tasks used for MVC are static co-contraction as well as dynamic calf rise and toe rise.

This signal after being converted into activation is used to drive a set of virtual muscle-tendon units. Activation is computed using the following equation to capture the non-linear twitch response of the muscle fiber:

$$
E(t)=\frac{\left(e^{A \bar{u}(t)}-1\right)}{\left(e^{A}-1\right)}
$$

With A being the EMG shape factor and $\bar{u}(t)$ being the filtered and normalized EMG.

To update the kinematic state of the virtual muscle-tendon units, the muscle-tendon length needs to be known. Muscle-tendon length cannot be experimentally recorded directly but can be computed as a function of joint angles using musculoskeletal geometry models. To speed up computation, we used a B-spline algorithm to compute muscle-tendon length from joint angles in real-time [21]. B-spline coefficients for each muscle-tendon unit are computed 
using values from the muscle analysis tool from OpenSim [25] where the full range of motion of each joint is sparsely explored.

A

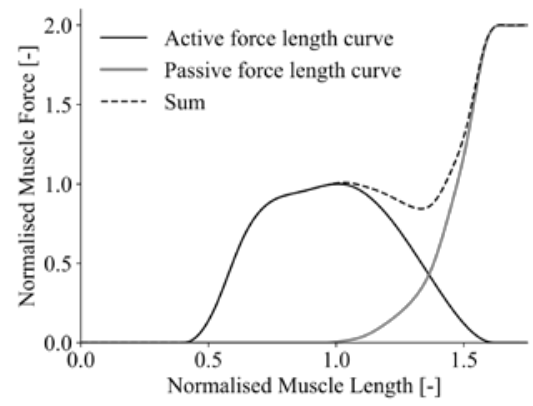

D

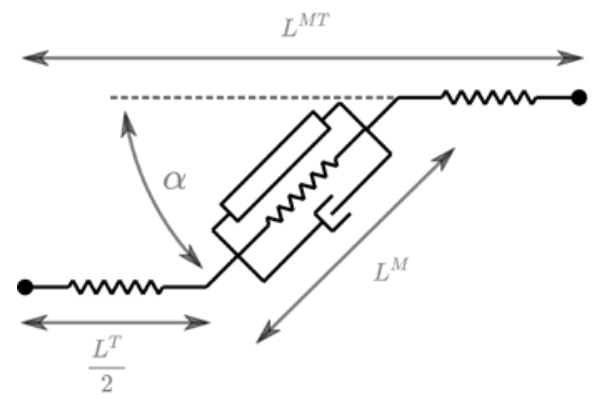

B

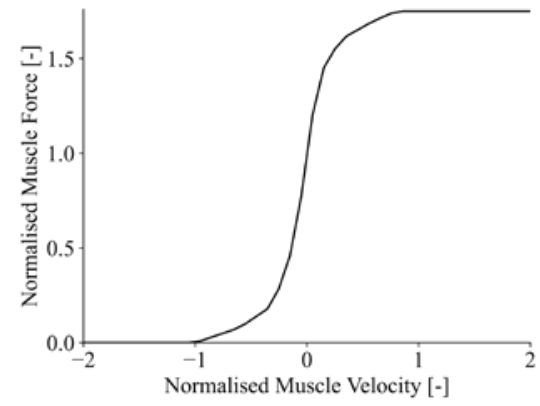

C

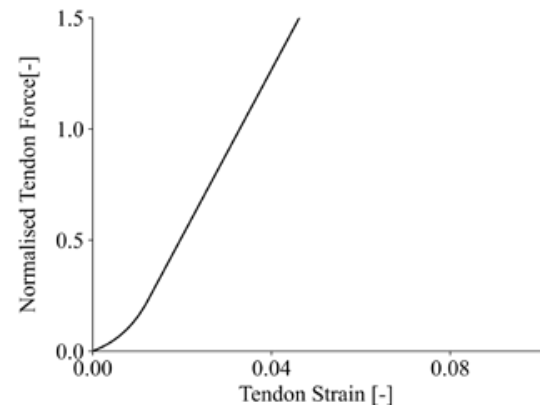

Figure 5-2: Normalized force curves and Hill-type muscle model used in the human-machine interface with the modelled A) muscle fiber active force length and passive force-length relationship, B) muscle fiber force-velocity relationship of the muscle, C) elastic tendon forcestrain relationship D) the Hill-type muscle representation.

From these values, muscle forces are computed using personalized Hill-type muscle models (Fig. 5-1-C, Musculotendon dynamics stage). This muscle model consists of a non-linear spring (tendon) in series with three elements representing the muscle fibers: an active contractile element, in parallel with a non-linear spring and a linear damper (i.e. muscle fiber passive elements).

Muscle-tendon force is computed using the following equation:

$$
F^{M T U}=F^{T}=F^{M} \cos (\alpha)
$$

With $F^{M T}$ representing the muscle-tendon force, $F^{T}$ is the tendon force, $F^{M}$ is the muscle fiber force and $\alpha$ is the muscle fibers pennation angle.

Muscle force is computed using the following equation: 

Repertoire of Locomotion Conditions

$$
F^{M}=F_{I s o}^{M a x}\left(\overline{f_{a, l}}\left(\overline{L^{M}}\right) \overline{f_{a, v}}\left(\overline{V^{M}}\right) E(t)+\overline{f_{p}}\left(\overline{L^{M}}\right)+D^{M} \overline{V^{M}}\right)
$$

With $F_{I s o}^{M a x}$ representing the maximal isometric muscle force, $\overline{f_{a, l}}\left(\overline{L^{M}}\right)$ is the active normalized muscle force-length relationship (Fig. 5-2-A), $\overline{f_{a, v}}\left(\overline{V^{M}}\right)$ is the active normalized muscle force-velocity relationship (Fig. 5-2-B), $\mathrm{E}(\mathrm{t})$ is the muscle activation, $\overline{f_{p}}\left(\overline{L^{M}}\right)$ is the passive normalized muscle force-length relationship (Fig. 5-2-A), $D^{M}$ is a linear damper, $\overline{L^{M}}$ is the muscle length normalized by $L_{O p t}^{M}, L_{O p t}^{M}$ the optimal fiber length represents the muscle length at which the muscle force production is maximal and $\overline{V^{M}}$ is the muscle velocity normalized by $10 * L_{O p t}^{M}$.

As previously stated, from joint angles the whole length of the musculotendon system is obtained but the muscle length is needed to compute muscle force. For this, the BrentDekker root-solver iterative method [139] is used for solving the equilibrium between muscle force and tendon force. Tendon force is obtained using the passive tendon force-strain relationship (fig. 5-2-C). The tendon strain is obtained using the following equation:

$$
S^{T}(t)=\frac{L^{T}(t)-L_{\text {Slack }}^{T}}{L_{\text {Slack }}^{T}}
$$

With $S^{T}(t)$ the tendon strain, $L^{T}$ is the tendon length and $L_{\text {Slack }}^{T}$ is the tendon slack length, which is the length at which the tendon does not produce force.

As dynamic contractions occur in the muscle, change in fiber kinematics results in pennation angle $\alpha$ change, while the overall muscle thickness is kept constant. Pennation angle $\alpha$ is continuously updated at each time instant t using the following equation:

$$
\alpha(t)=\arcsin \left(\frac{L_{O p t}^{M} \sin \left(\alpha_{O p t}\right)}{L^{M}}\right)
$$

Finally, muscle forces are projected onto the ankle joint plantar-dorsiflexion degree of freedom via the moment arms to obtain joint torque. The moment arm is obtained via the partial derivative relative to joint angles using the B-spline algorithm previously introduced.

\subsubsection{Model personalization}

A generic musculoskeletal geometry model is scaled linearly to each individuals using the open-source software OpenSim [25] and 3D motion capture data of body landmark (bony area) recorded during a static pose. During this procedure, muscle insertion points and wrapping points are linearly moved to offer personalized muscle-tendon length and muscle moment arm. This scaled model is the one used to create a multidimensional BSpline function per muscle-tendon unit. After model scaling, the muscle-tendon model needs to be personalized to the subject using non-linear calibration to capture subject properties that are 
varying non-linearly across individuals. Four parameters are calibrated for each muscle in the model, A the EMG shape factor, $L_{\text {Slack }}^{T}$ the tendon slack length, $L_{O p t}^{M}$ the optimal fiber length and $F_{I S O}^{M a x}$ the maximal isometric muscle force. This calibration is based on two part. First a pre-tuning [56], which is identifying initial values for the optimal fiber length $L_{O p t}^{M}$ and tendon slack length $L_{\text {Slack }}^{T}$ using an optimization procedure (interior point optimizer) [140] that minimize the following equation:

$$
\min \sum_{i=1}^{11}\left(L^{M T}(i)-L_{P r e c}^{M T}(i)\right)^{2}
$$

With $L^{M T}(i)$ the muscle-tendon length from the scaled model, i eleven angles equally spaced across the full range of motion of the DOFs crossed by the considered muscle and $L_{\text {Prec }}^{M T}(i)$ the estimated muscle-tendon length computed using the following equation:

$$
L_{\text {Prec }}^{M T}(i)=L_{\text {Slack }}^{T}+L_{\text {Slack }}^{T} \varepsilon_{T}(i)+L_{\text {Opt }}^{M}+\overline{L^{M}}(i) \cos (\alpha(i)
$$

With $\overline{L^{M}}(i)$ the normalized muscle length determined from the unscaled model and $\varepsilon_{T}(i)$ computed using the following equations:

$$
\begin{gathered}
\varepsilon_{T}=\frac{\overline{F^{m}} \cos (\alpha)+0.2375}{37.5} \text { for } \varepsilon_{T}>0.0127 \\
\varepsilon_{T}=\frac{\ln \frac{\overline{F^{m}} \cos \alpha}{0.06142}+1}{124.929} \text { for } \varepsilon_{T} \leq 0.0127
\end{gathered}
$$

The second part is a calibration of all of the four parameters based on optimization using simulated annealing [57] that minimizes the error between the torque estimated by the model and the experimental torque computed for different motor tasks (Fig. 1-F):

$$
\sum \sum \frac{1}{N_{\text {Rows }}} \sum\left(\tau_{\text {Pred }}^{N_{\text {Trials }}}-\tau_{I D}\right)^{2}
$$

With $N_{\text {Rows }}$ the number of data points for the considered trials, $\tau_{\text {Pred }}$ the joint torque computed by our model presented in the previous section and $\tau_{I D}$ the experimental joint torque computed using inverse dynamics using the inverse dynamics tool from OpenSim, which uses experimental joint angle and ground reaction forces.

The parameters boundaries are for the EMG shape factor A varying between -3 and 0 , for the maximal normalized isometric muscle force $F_{I S O}^{M a x}$ varying between 0.5 and 1.5 and for the optimal fiber length $L_{O p t}^{M}$ varying between $\pm 2.5 \%$ and tendon slack length $L_{\text {Slack }}^{T}$ varying between $\pm 5 \%$ from the values found during pre-tuning. 


\subsubsection{Bi-lateral ankle exoskeleton}

In this study, the ankle modules of the Symbitron exoskeleton were used [33] to assist plantar-dorsiflexion during locomotion tasks. Due to the modularity of the exoskeleton, and its possible 10 degree of freedom configuration, the ankles are relatively heavy at $5 \mathrm{~kg}$ each. Each ankle exoskeleton has two degrees of freedom: active plantar flexion and dorsiflexion, and passive inversion and eversion. The active degree of freedom is actuated with a rotary series elastic actuator (SEA), which transmits the desired interaction forces via a push-pull rod from its distal location to the ankle joint. The SEA consists of a motor (Tiger Motor U810(Pro), T-Motor, Nancheng, China) that is connected to a harmonic drive (LCSG20, Leader Drive, Jiangsu, China) with a gear ratio of 1:100. The harmonic drive is connected to the output of the motor with a custom rotary spring with a stiffness of $1534 \mathrm{Nm} / \mathrm{rad}$. The actuator can deliver a controlled peak torque of $100 \mathrm{Nm}$ and has a maximum output speed of $5 \mathrm{rad} / \mathrm{s}$. The motor is controlled via an Everest Net drive (Ingenia, Barcelona, Spain), which communicates with the control computer via EtherCAT. Motor position is measured via a rotational encoder (16 b MHM, IC Haus, Bodenheim, Germany). Additionally, the actuator measures the spring deflection and joint position with two encoders $(20 \mathrm{~b}$ Aksim, RLS (Renishaw), Kemnda, Slovenia) which are transmitted to the control computer via the Everest Net drive. The backpack contains the control computer, a NUC (Intel, Santa Clara, USA) that executes the controller in TwinCAT 3 (Beckhoff Automation, Verl, Germany) in real-time with a sampling frequency of $1 \mathrm{kHz}$. Additionally, the backpack contains two batteries, supplying the computer and actuators with power. The backpack has a weight of $10 \mathrm{~kg}$.

\subsubsection{Low-level torque control via disturbance observers}

The Symbitron ankle exoskeleton interacts with its subject by controlling the interaction torque between subject and exoskeleton, i.e. the spring torque $\tau_{\text {exo }}$. This torque is computed from the measured spring deflection and a linear model of the actuator's spring (Fig. 5-1-E). The interaction torque is controlled via a disturbance observer algorithm previously developed for lower limb exoskeletons [141]. The controller fulfils three purposes: it increases the bandwidth of the actuator to $30 \sim \mathrm{Hz}$, it lowers the actuator's apparent impedance, i.e. it makes the actuator as mechanically transparent as possible, and it guarantees unconditional interaction stability with any environment. The latter is especially important to avoid instabilities during ground contact. The controller consists of an inner loop PD controller with feedforward term, that increases the actuator's torque bandwidth, and an outer loop disturbance observer (DOB) that lowers the actuator's apparent impedance.

The DOB computes the torque caused by disturbances, such as impacts during heel strike, or voluntary motion of the subject, and subtracts that disturbance torque $\tau_{\text {dist }}$ from the desired reference torque $\tau_{\text {support }}$, which is the estimated joint torque from the neuromusculoskeletal model multiplied by a support ratio, to eliminate the effect of the 
disturbance on the interaction torque $\tau_{k}$. This disturbance rejection makes the actuator as transparent as possible while keeping its interaction with the environment stable. The resulting torque $\tau_{m}$ is sent to the motor as a reference.

\subsubsection{Assistance}

To assure timely and voluntary assistance a tight integration between high and low-level control is required. For this, the estimated joint torque is sent from the model to the lowlevel controller via the Ethercat real-time communication protocol where it then multiplied by a support ratio. This support ratio varies between 0 and 1 with 0 meaning that the exoskeleton acts in minimal impedance and 1 signifying 100\% of assistance given (i.e. the exoskeleton assists with the same amount of torque as the subject's biological joint). The Assistance is then thresholded to a maximum of 30 N.m to assure the security of the subject and the integrity of the actuator.

\subsection{Experiment}

Experiments were conducted on 3 subjects $(31 \pm 5$ years, $177 \pm 7.5 \mathrm{~cm}, 70.3 \pm 7.3 \mathrm{Kg}$ ). Participants had no instance of musculoskeletal injury or motor-control impairment. All experimental procedures were carried out in accordance with the Declaration of Helsinki on research involving human subjects. All subjects provided their explicit written consent to participate in the study.

The experiment started with the personalization phase. Motion capture's 3D markers data (Oqus, Qualisys, Sweden), ground reaction force (M-Gait, MotekForce Link, The Netherlands) and EMG (AxonMaster 13E500, Ottobock, Germany) were recorded on the subject. EMG signals for eight muscles were recorded, left and right Soleus, Tibialis Anterior and Gastrocnemius Medialis and Lateralis. Marker data and ground reaction forces were used to compute joint angles and joint torques using the inverse kinematics and inverse dynamics tool from OpenSim [25]. This data was further used to personalize the model using the methods described in section 5.2.2 (Model personalization). The model used during this experiment was based on [25] and contains the following joints: left and right plantar dorsiflexion and knee flexion-extension and the following 14 muscles tendon unit: left and right Soleus, Tibia Anterior, Gastrocnemius Medial and Lateral and Peroneus Longus, Brevis and Tertius. The following tasks were used for the calibration of the model: static, treadmill walking at $1.8 \mathrm{~km} / \mathrm{h}$ and $2.8 \mathrm{~km} / \mathrm{h}$, calf rise and toe rise.

The second phase of the experiment, after model personalization, consisted in defining the experimental parameters and acclimation of the subject to walking with the exoskeleton. Two experimental parameters were set during this phase, the support ratio (see Table 5-1) and the steps frequency for the two tested speeds. Step frequency was controlled during the experiment to make sure that assistance is integrated by the subject and reduce his own torque generation instead of increased speed or step length. The support ratio was selected by the subjects to be a trade-off between comfort and high assistance. Furthermore, since 


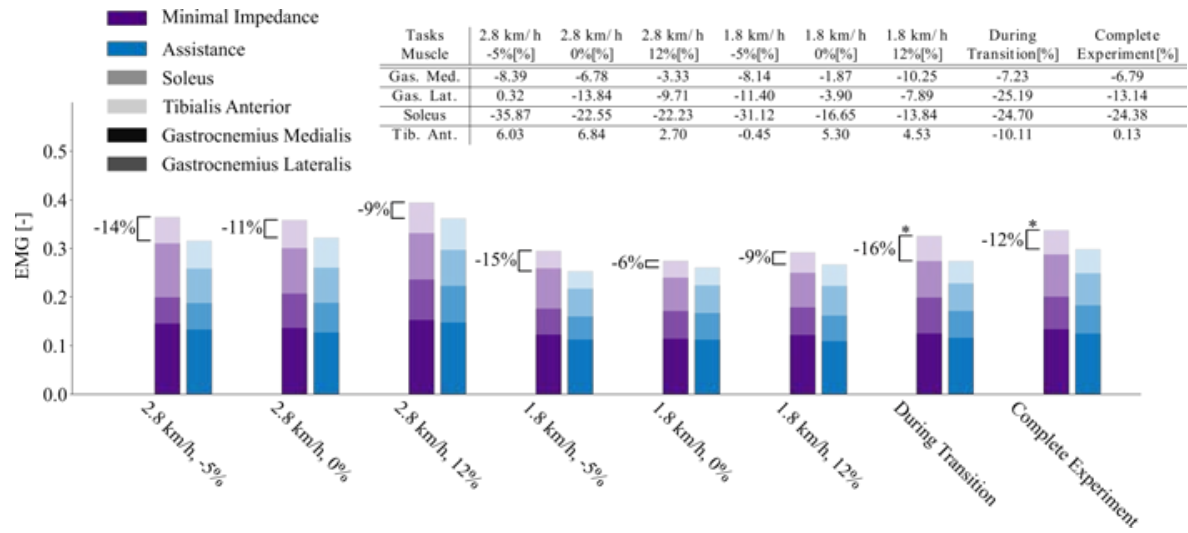

Figure 5-3: Mean EMG reduction for all subjects within each locomotion condition as well as during transitions and during the entire experiment (i.e. all conditions and transitions altogether). Data are reported for the assisted and non-assisted condition. The $*$ represents statistical significance $(\mathrm{P}<0.05)$. Individual EMG reduction results for each muscle for all tasks, transition and complete experiments presented in the table.

most of the participants were naive subjects, training was necessary to eliminate learning effects during the experiments and get acclimated to walk with the exoskeleton. Previous work has shown that at least 45 min of walking is needed to obtain full metabolic benefice of the assistance and normal joint kinematics [142]. For this, the subjects walked on a treadmill (Thera-Treadpro, Sportplus) with the exoskeleton with and without assistance until

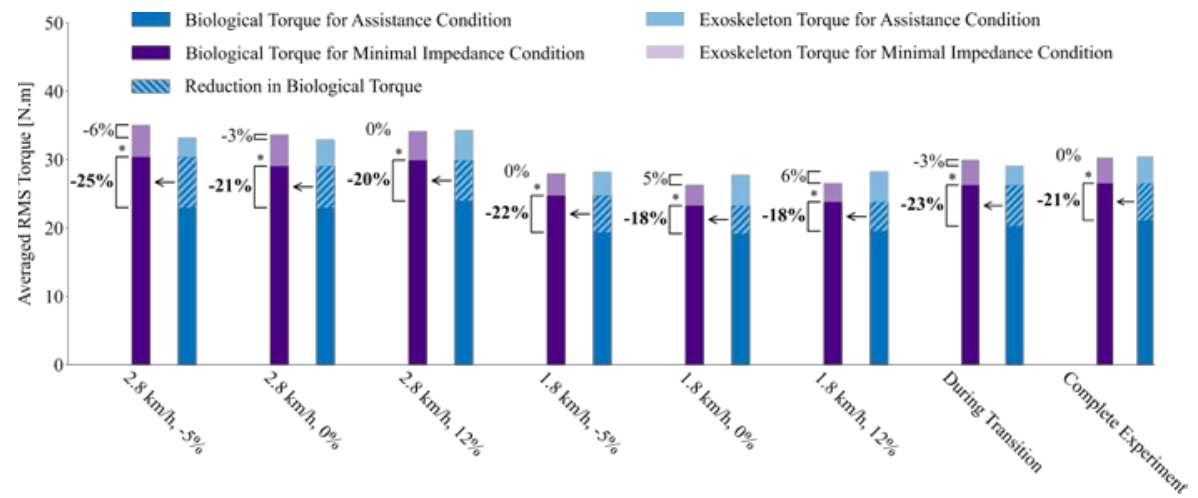

Figure 5-5: Ankle joint torque root mean squared sum averaged across all subjects within each tested condition, i.e. assisted (in blue) and non-assisted (minimal impedance, in purple). The darker color represents the human biological torque estimated via the proposed HMI. The lighter color represents the exoskeleton torque recorded by the spring deflection of the actuator. The dashed area represents the portion of human biological ankle torque that is taken over by the exoskeleton. The number on top of each bar represents the difference in percentage of total ankle torque (exoskeleton and biological) between conditions. The lower number in bold represents the reduction in biological torque between the conditions with * representing statistical significance $(\mathrm{P}<0.05)$. 
they presented a natural looking gait and were feeling confident (10 to 20 minutes). To access knee joint angles, an IMU suit was used (Link, Xsens, the Netherlands), the ankle joint angle was directly available from the joint encoder of the exoskeleton. The knee angles are required for the gastrocnemius muscles that span the ankle and knee joint.

The last phase of the experiment was the recorded experiment. Two conditions were tested, minimal impedance and proportional estimated torque assistance provided by the model. Each condition was tested with six different locomotion tasks that were randomly presented to the subject. The data was collected in a single uninterrupted session including the six randomly combined conditions and the transitions within. The tested locomotion tasks were $1.8 \mathrm{~km} / \mathrm{h}, 0 \% ; 1.8 \mathrm{~km} / \mathrm{h},-5 \% ; 1.8 \mathrm{~km} / \mathrm{h}, 12 \% ; 2.8 \mathrm{~km} / \mathrm{h} \mathrm{0} \%, 2.8 \mathrm{~km} / \mathrm{h},-5 \% ; 2.8 \mathrm{~km} / \mathrm{h} \mathrm{12 \%}$. Each task had a duration of $3 \mathrm{~min}$, the transition between tasks was of variable length due to the time needed by the treadmill to change between speed and/or inclination. A fall prevention system was used every time the subjects were walking with the exoskeleton (ZeroG, Aretech LLC, USA) which provided a body-weight support of $5 \mathrm{Kg}$.

\subsection{Results}

In most figures, we first present each individual tested walking tasks followed by "during transitions", which is the average of all transition happening between two walking tasks (i.e. change in speed, elevation or both) and finally, the "complete experiment", which represents the full recorded experiment with walking task and tasks transition.

\subsubsection{Data processing}

All data were segmented automatically using a peak detector on the knee joint angle. Each segment was re-sampled as percentages of the gait cycles (from 0 to $100 \%$ ). The root mean
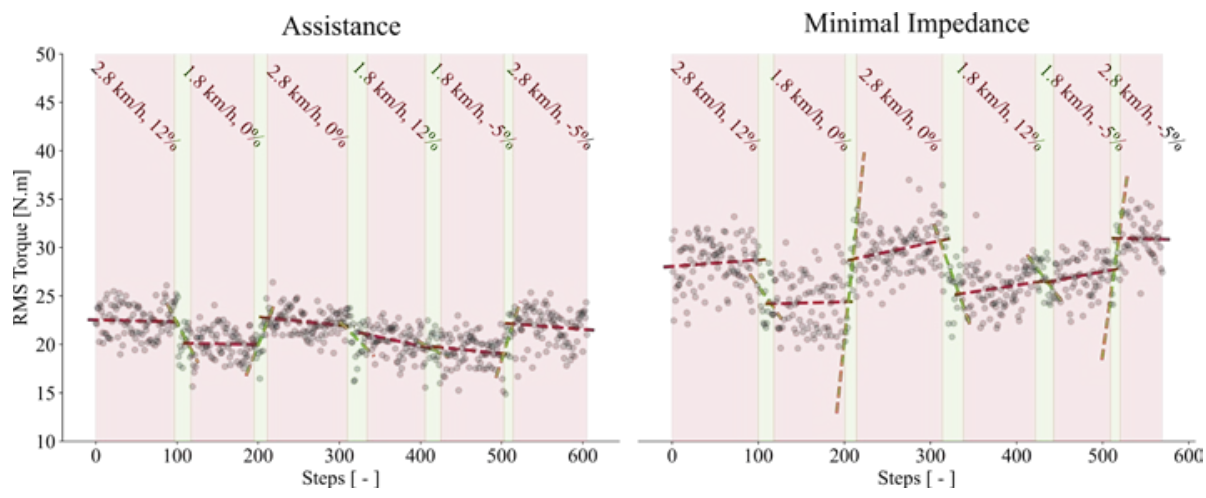

Figure 5-4: Estimated joint ankle torque for a representative subject for two tested conditions, i.e. assisted and non-assisted (minimal impedance). Each grey dot represents the torque root mean squared sum (RMS) for each gait cycle. The red dotted line represents torque RMS trend within each locomotion condition. The green dotted line represents torque RMS trend during transitions across conditions.

squared value was identified for each segment, values that were superior to three times the 
interquartile values were removed. Finally, percentages of change between conditions (assistance and minimal impedance) were calculated using the mean of all steps. Significance was computed using linear mixed modelling (mixedlm from the python statsmodels library).

\subsubsection{EMG reduction}

For the EMG, Fig. 5-3 shows that reduction could be achieved for all tasks as well as during the transition between tasks (16\%, significant) and for the complete experiment $(12 \%$, significant). Fig. 5-3 presents detailed results at the muscle level. It can be observed that reduction was obtained for all muscles and all tasks, during transitions and for the complete experiments (maximum reduction of $35 \%$ for the soleus at $2.8 \mathrm{~km} / \mathrm{h}$ with an inclination of $-5 \%$ ) except for the Tibialis Anterior that did not undergo reduction (except during task transition $(10 \%)$ and walking at $1.8 \mathrm{~km} / \mathrm{h}$ with $-5 \%$ inclination $(0 \%))$ and for the Gastrocnemius lateralis at $2.8 \mathrm{~km} / \mathrm{h}$ with an inclination of $-5 \%(0 \%)$. Table $5-1$ presents the EMG reduction obtained for each subject independently. It can be observed that the Soleus muscle always presented reduction in EMG superior to 5\% (blue cell color) for all subjects (maximum $40.6 \%$ (subject 1 for $2.8 \mathrm{~km} / \mathrm{h},-5 \%$ ) and minimum $9.5 \%$ (subject 1 for $1.8 \mathrm{~km} / \mathrm{h}$, $12 \%)$ ). The tibialis anterior is the muscle presenting the least reduce EMG level with increase always superior to $5 \%$ (red cell colour) for subject 1 .

\subsubsection{Biological Torque Reduction}

Fig. 5-4 shows the estimated biological ankle torque averaged for each gait of a representative recording for the two tested conditions (i.e. minimal impedance and assistance from the model based HMI) as well as the trends (dashed line) for the tested tasks (red) and during the transition (green).

Fig. 5-5 presents the estimated biological ankle torque for all the tested conditions as well as during the transition and in the complete experiment between the two conditions. Reductions were achieved for biological ankle torque for all tested condition (significant) (ranging from $18 \%$ to $25 \%$ ) and reduction of $23 \%$ (significant) was obtained also during the transition between tasks. The final reduction in ankles for the complete experiment was $21 \%$ (significant).

Table 5-1 presents ankle torque reduction obtained for each subject independently. It can be seen that for all subject and all condition the reduction in biological torque was always greater than $5 \%$ (blue cell color) (maximum 36.2\% (subject 1 for $2.8 \mathrm{~km} / \mathrm{h},-5 \%$ ) minimum $8.6 \%$ (subject 2 for $1.8 \mathrm{~km} / \mathrm{h}, 12 \%)$ ). Table 5-1 also shows the averaged assistance in N.m. received by subjects and the individual support ratio. 
Table 5-1: Subject specific support ratio, EMG and torque reduction and averaged received assistance for all tasks, transition and complete experiments. Red cell represents increase greater than $5 \%$, blue cell represents reduction greater than $5 \%$ and grey cell represents reduction between $-5 \%$ to $5 \%$.

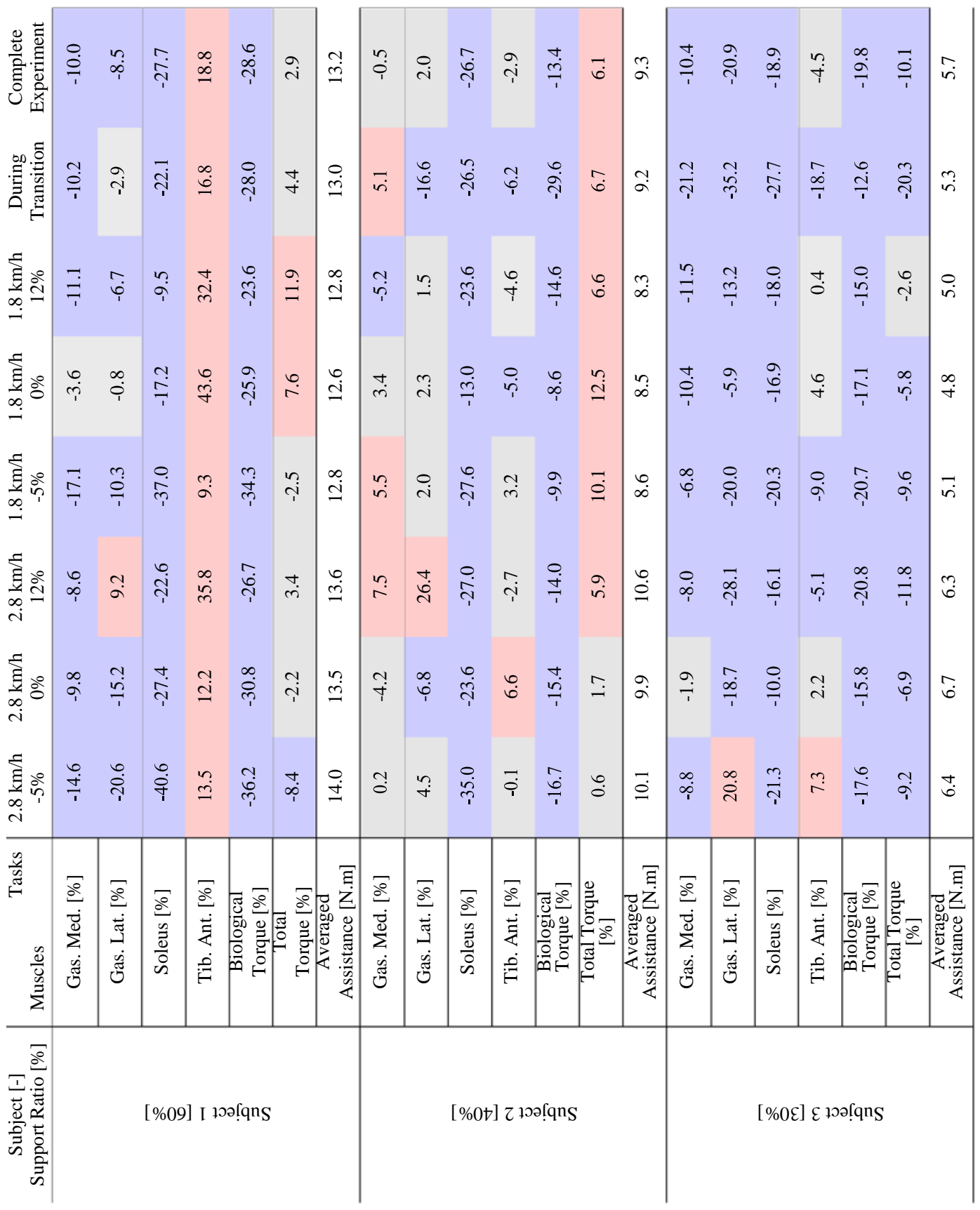




\subsubsection{Human-exoskeleton Torque invariance across conditions}

The total human-exoskeleton torque (summation of the estimated biological ankle joint torque and exoskeleton torque) was preserved across tasks (between $-6 \%$ for the $2.8 \mathrm{~km} / \mathrm{h}$ at $-5 \%$ inclination and $6 \%$ for $1.8 \mathrm{~km} / \mathrm{h}$ at $12 \%$ inclination) as well as for the complete experiment $(0 \%)$. Table 5-1 shows the human-exoskeleton torque per subjects.

\subsection{Discussion}

In this paper, we presented a novel HMI to assist during a wide range of locomotion tasks. We based our HMI on a high-level neuromusculoskeletal model controller driven by EMGs and joint angles. The low-level control was based on an efficient torque controller based on a disturbance observer connected to a bilateral ankle exoskeleton to give continuous and volition assistance. Results from Fig. 5-5 and 5-3 show that the developed HMI can positively assist for all tested locomotion tasks.

More importantly, the developed HMI can assist positively during transitions between tasks without having to change control parameters or having to switch across assistance profiles. This is visualized in Fig. 5-4, where different torque profiles are dynamically generated by our proposed HMI across different locomotion tasks, prescribing more torque to the exoskeleton for more demanding tasks (higher speed and inclination). Exoskeleton dynamic adaptation to different locomotion condition is central for enabling wearable exoskeleton to be employed outside of the lab in rough and unstructured terrains. The presented HMI allows capturing how an individual's musculoskeletal function varies across movements, thereby generating exoskeleton control commands, something that state of the art exoskeleton HMIs cannot do. Human-in-the-loop optimization of assistive torque profiles needs up to $45 \mathrm{~min}$ [30], during which the human subject is exposed to sub-optimal torque patterns. In contract, the optimization-based HMI calibration employed in our proposed approach is done once per subject and requires only a few minutes of locomotion data, i.e. 2 minutes on averages per subject.

Our proposed approach showed the ability to extrapolate outside of the calibration condition (i.e. over-bound locomotion at $1.8 \mathrm{~km} / \mathrm{h}$ and $2.8 \mathrm{~km} / \mathrm{h}$ ), thereby enabling positive assistance during unknown locomotion speeds, ground elevations and transitions. Importantly, no exoskeleton was worn during the recording of calibration data, showing the approach could compensate for the added exoskeleton during the real-time control tests. This shows that, contrary to current controllers based on state machines, the presented HMI can achieve tasks-independence that enables exoskeleton voluntary control, which offers positive assistance (i.e. reduction of biological torque and EMG).

These advantages were previously demonstrated by our previous works where the HMI can estimate joint torques in real-time for a wide range of tasks such as walking, backward locomotion, calf rise, jumping, sidestepping and calf rising [79]. Furthermore, it offers further advantage than the ability to predict joint torques on a wide range of tasks, it also offers great 
flexibility as it can easily be adapted to other body parts and wearable robotic devices such as upper-limbs, which we demonstrated when the proposed HMI was used to control a hand prosthesis in an amputee [18] and assisted via a soft exosuit, elbow's dynamic movement [143]. This method was also be applied successfully to post-stroke and spinal cord injury patients to voluntary control their knee and ankle joint simultaneously [144].

Another interesting result is that the total amount of joint torques needed to walk (humangenerated + exoskeleton-generated torque) were preserved throughout the entire experiment. This can be seen in Fig. 5-5 in the summation of estimated joint torque and interaction torque. This shows that a synergistic transfer of torque happened between the robotic device and the subject (hashed area in Fig. 5-5) where the torque provided by the exoskeleton results in the same amount of biological torque being reduced in both left and right ankle joints across subjects. In this way, the human and the exoskeleton converged towards a condition where locomotion was more economical in terms of EMG and biological torque.

This is due to the voluntary and natural assistance provided that is directly proportional to the subject's torque generation, which allows a precise and timely torque delivery by the exoskeleton. This could be achieved because the real-time EMG-driven model can predict joint torque before the subject's muscle contraction due to the electromechanical delay as shown in our previous publication [79]. Reduction of torque and EMG achieved in our experiments during a complex task such as locomotion could only be attained with an underlying torque delivered by the exoskeleton in sync with muscle contraction. Timely torque delivery has been shown to be crucial for metabolic reduction notably during the push-off phase [145]. Metabolic comparison via respiratory systems will be conducted in future work to compare net metabolic reduction. Future work will also test our proposed HMI to control a more lightweight exoskeleton in an unconstrained environment to fully explore the benefit of this HMI out of the lab.

One of the limitations of the current method observed during the experiments was occasional instability during swing phase. As stated previously, the assistance provided is proportional to the subject's joint torque and thus assistance is provided continuously. Assisting only the push-off phase would have required implementation of a classification algorithm, limiting the continuous and task-independent aspect of our HMI. This came with limitation, notably, assistance during swing where the ankle joint is less stiff than during pushoff and thus can easily be perturbed by an external force applied to it (i.e. assistance provided by the exoskeleton).

Future work will try to remove ankle instability during swing phase by modulating the support ratio based on joint stiffness estimation, to provide more assistance when the joint is stiff (i.e. push-off) and less assistance when the joint is lax (i.e. swing) [46], [146]. 
Future work will also investigate high-density EMG and blind-source separation to directly establish a connection between the muscle model and spinal motor neurons [71], [121], [147]. This will add robustness for the placement of EMG due to the high density of spatial distribution of EMG sensors. This will also remove the need for maximal voluntary contraction for the normalization of EMG signal since the motor unit transmits information in the frequency domain and not in the amplitude domain.

We will also investigate learning effects and time needed for the subject to fully adapt to the assistance and compare them with other states of the art HMI like human in the loop controller.

\subsection{Conclusion}

We presented an HMI that enabled different individuals to control a robotic bi-lateral exoskeleton voluntarily during a broad range of locomotion conditions and transitions. The proposed approach enabled exoskeleton automatic adaptation to unknown locomotion conditions that were not considered during the HMI design. Moreover, the controlled exoskeleton dynamically adapted to different conditions with no need to use locomotion mode classification or state machines. This is an important step to enable the use of wearable robots outside of the lab during real-life situations. 


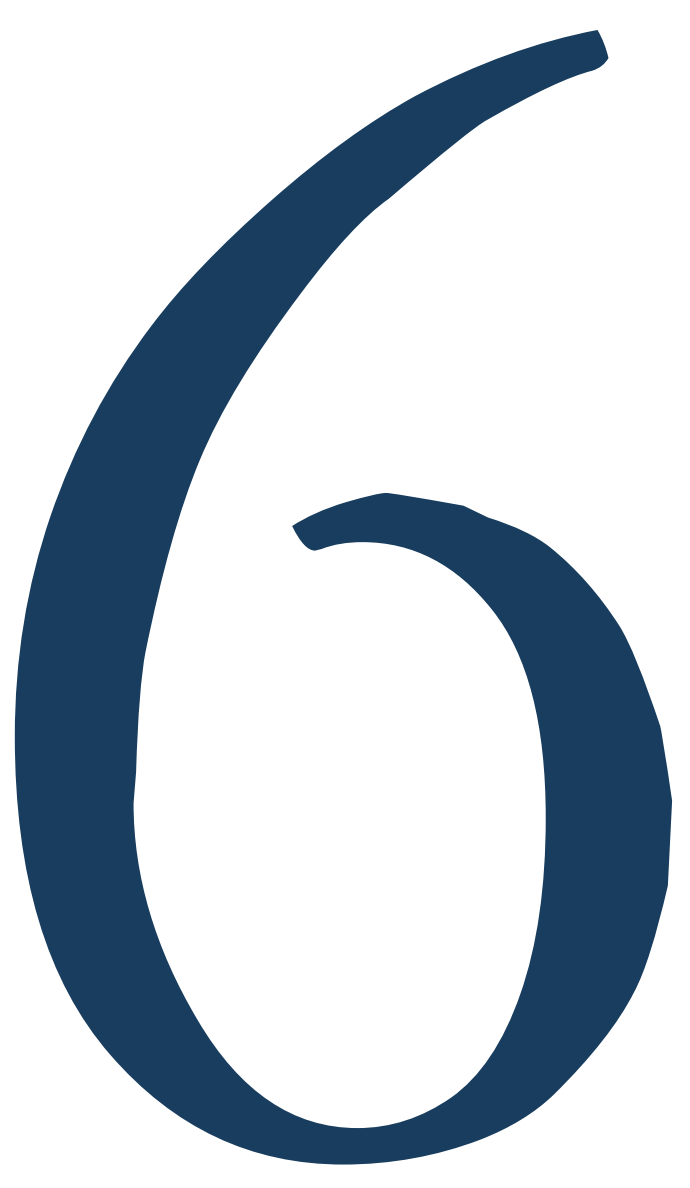




\section{CONCLUSION}

\subsection{Summary of Key Findings}

We created a new tool that allows the computation of musculoskeletal variables such as muscle forces and kinematics as well as joint torques in real-time while moving with a wearable robotic device. We also showed that we can use this information to provide robotic assistance to healthy users as well as stroke and spinal cord injury patients.

We first showed that EMG-driven modelling can be used in real-time and gave results (i.e., joint torques) close to the gold-standard (inverse dynamics). In the second chapter, results indicated a root mean squared error (RMSE) between estimated joint torques from our HMI and experimental joint torques from inverse dynamics of $0.17 \mathrm{Nm} / \mathrm{kg}$ and a Person coefficient of $r=0.65$ for the knee flexion-extension, an RMSE of $0.17 \mathrm{Nm} / \mathrm{kg}$ and $\mathrm{r}=0.74$ at the ankle plantar-dorsiflexion and $0.05 \mathrm{Nm} / \mathrm{kg}$ and $\mathrm{r}=0.61$ at the ankle subtalar flexion. These results show that we can answer positively to our first research question:

1) Can real-time EMG-driven modelling achieve accuracy in joint torques close to the golden standard (Inverse dynamics)?

In Chapter 2, we also confirmed that internal body variables such as joint torques, muscle forces, and muscle kinematics can be calculated within the electromechanical delay (EMD) ( $<50 \mathrm{~ms}$ ). Results showed computation from EMG to joint torques under $35 \pm 11 \mathrm{~ms}$. This is essential for controlling exoskeletons where the assistance timing is crucial [30]. This result shows that we can answer yes to our second research question:

2) Can real-time EMG-driven modelling achieve computation time within EMD?

For improving the mobility of patients, it is important to enable them to perform many different tasks independently. Thus it is fundamental to be able to assist with a robotic device for a large repertoire of tasks. In the second and fifth chapters, the extrapolation capabilities of our framework, namely, computing biomechanical variables on conditions not used for calibration, were presented. In the second chapter, the results showed that accurate joint torques were computed with respect to inverse dynamics on unknown tasks (fast walking, backward walking, sidestep, single-leg squat, and single-leg calf rise) and on uncalibrated degrees of freedom (DOF) (subtalar joint). These results demonstrated the extrapolation capabilities of our HMI. Furthermore, in the fifth chapter, computation of joint torques on unknown tasks (elevation) and when the users were assisted by an exoskeleton, were demonstrated. These results were not validated against inverse dynamics. The controlled exoskeleton reduced the users' EMG and biological joint torque levels, which may not happen with non-physiological assistance. When assisted by non-physiological assistance, users may consider it as perturbations and thus co-contract muscles. These results on 
exoskeleton demonstrated, once more, the extrapolation capabilities of our HMI. These results indicate that positive answers can be addressed to our second research question:

3) Can EMG-driven modelling extrapolate results outside of its calibration data?

Providing assistance during locomotion task needs good timing and efficient force transmission. This was the main focus of chapter 5. Here, we demonstrated that for healthy users we can deliver variable assistance for different locomotion modalities (change in speeds and/or inclinations), which produced reductions in EMG levels. This was particularly challenging as the assistance had to be properly timed with properly estimated joint torques. Our study showed a reduction in EMGs ranging from $6 \%$ to $18 \%$ for the tested tasks as well as a reduction of $16 \%$ for the transition periods in between tasks and $12 \%$ for the total. We also found a reduction in biological ankle torques ranging from $19 \%$ to $25 \%$ for the tested tasks as well as a reduction of $23 \%$ for the transition periods in between tasks and $21 \%$ for the total. Importantly, the total user + exoskeleton ankle joint torques between conditions (minimal impedance and assistance) were identical. This means that, between the user and the exoskeleton, a force transfer happened proving the natural and voluntary assisting components of the HMI based on EMG-driven modelling. These results show that we can answer positively to our third research question:

4) Can predicted joint torques from EMG-driven modelling offer reduction in EMG and joint torques levels when used to assist via an exoskeleton healthy users during diverse locomotion modalities?

Although most of the experiments in this dissertation were done on healthy users to validate the HMI's safety and feasibility, it was also tested on patients in Chapter 4. In general, paretic patients are a more difficult group to test with, but it is meaningful to do so because the assistance of paretic patients is the main aim of assistive robotics for rehabilitation. In this chapter, assistance was given to a group of patients (two strokes patients and one spinal cord injury (SCI) patient) through the H2 exoskeleton [144] with our HMI. We investigated seated tasks close to the ones used during rehabilitation exercises. Patients' neuromusculoskeletal (NMS) models were calibrated directly using the exoskeleton. This was needed as most patients cannot accomplish the tasks needed for dynamic calibration (i.e., walking, calf rise and so on.. ). Results showed that patients assisted by our HMI and the exoskeleton can track reference trajectories with good accuracy (tracking error always $<5.5 \pm 3.1$ degrees and with correlation coefficients always $>0.6$ ). These results show that we can answer positively to our fourth research question:

5) Can the developed HMI allow paretic patients to voluntarily control a robotic device? 
An easy way to increase mobility in patients is to render locomotion more economical. This can be validated by checking EMG reductions with the developed HMI. From chapter 4, results on patients showed that we can reduce the EMG level between different levels of assistance. For stroke patient 2, we obtained a reduction in EMG from (Low Gain) 0.69 \pm 0.14 to (High Gain) $0.36 \pm 0.14$ and a decrease in EMG variability from 1.24 to 0.67 (normalized from 26 to 21). For the SCI patient, we obtained a reduction in EMG from $0.90 \pm 0.18$ to $0.89 \pm 0.005$ and a decrease in EMG variability from 0.83 to 0.58 (normalized from 6 to 4.2)). These results provided an encouraging answer to our last research question:

6) Can assistance via a robotic device based on EMG-driven modelling provide EMG amplitude and variability reductions in paretic subjects?

\subsection{Impact and Relevance of the Scientific Contributions}

The developed HMI in this dissertation, for the first time, predicted joint torques of multiDOFs in real-time and with good accuracy. The developed HMI is compatible with fully wearable sensors (EMG and IMU-suit) (chapters 3 and 5) and reduces the time between data recording and data analysis (chapter 2). These features allow the developed HMI to also make an impact outside of the research lab.

Achieving a computation time less than the EMD is important as this allows estimation of the internal neuromechanical variables of the subject before their physical realizations (chapter 2). This can be a game-changer for assistive robotics where, with the help of robotic devices, we can steer the neuromechanical properties toward a more favourable area (i.e. plateau of the muscle force-length relationship) and reduce the muscular efforts for users. This can also help to prevent musculoskeletal injury. Furthermore, this real-time HMI can assist rehabilitation even without robotic help as the musculoskeletal properties can be given as bio-feedback to the patient directly or to the physiotherapist to evaluate the effects of different exercises. For instance, the HMI can capture the patient's impaired muscular forcegenerating capacity (chapter 4), thus, helping clinicians to refine personalized neurorehabilitation solutions.

The possibility of our HMI to extrapolate beyond the calibration data is also valuable for assistive devices (chapters 2 and 5). There are currently no robotic controllers that can continuously accurately predict the state of the users. Most controllers require the use of a state machine type of controller [29] or do not accurately predict users' state (i.e., uncalibrated model and without the use of experimental EMG). The assistance computed from our HMI can continuously assist the users in different conditions (speeds, inclinations) as well as in between tasks where the locomotion's modality changes. To our knowledge, this has never been reported before. Moreover, since our assistance is directly proportional to the users' joint torques, exoskeletons can provide natural assisting torques which automatically change along with users' actions. This is vital for the neurorehabilitation of patients where the goal is to improve the patients' independent mobility. 


\subsection{Limitations and Directions for Future Research}

There exist several limitations in this work that can lead to interesting future works.

One important limitation lies in the current calibration process, which may include too much constraint for patients. The current gold-standard for model calibration requires calibration on dynamic tasks such as walking, calf rise or squat. Unfortunately, these may not be always feasible for some paretic patients and isometrics calibration (as used in Chapter 4) may not be valid for locomotion tasks. One possible way to solve this issue is to use on-line calibration where the patient will be assisted by an exoskeleton. Using real-time inverse dynamics, the patient's experimental joint torques could be extracted and used for calibration. One of the problems of this solution is that inverse dynamics require ground reaction forces, which, as explained in the introduction, cannot be recorded via wearable sensors yet. In another solution direction, it will be interesting to fully explore the interpolation possibility of isometrics based calibration on dynamics tasks.

Another difficulty is to assess the validity of the calibrated parameters such as maximal isometric force, optimal fiber length, and tendon slack length. The calibration results are currently only validated against joint torques from inverse dynamics. These values could be deducted (for the maximal muscle force using the physiological cross-sectional area (PCSA) [148] for example) or better constrained (optimal fiber length and tendon slack length) from magnetic resonance imaging (MRI) [149] or ultrasound. Research is being conducted toward this goal but MRI processing is still largely done manually, which could be difficult to generalize for all calibrations of the neuromusculoskeletal models and thus automatic methods should be developed. Statistical methods and population-derived modelling could also be interesting solutions [150], [151].

The calibrated neuromusculoskeletal model suffers also from limitations, not by the model itself but by the missing experimental validation of the predicted muscle forces. Experimental muscle force measurements are extremely difficult and constraining as the gold-standard method consists of surgical procedures to implement sensors [152]. Lately, a new device with less constraint has appeared that can inform about the shape of tendon forces [153]. This device can estimate muscle forces by tapping the tendon and measuring the speed of the shear wave propagation. This may be an easier way to validate muscle forces during dynamic tasks. This new tool could be a nice addition to the validation of the model and also for its calibration as these experimental values could add further information for calibrating of muscle parameters.

Furthermore, since our HMI is based on an EMG-driven modelling approach, accessing healthy EMGs is mandatory. However, this can be difficult when working with patients, for example, complete SCI patients may not have EMG signals anymore or in stroke patients some muscles do not have EMG signals (i.e., patients with drop foot syndrome for example) 
or are perturbed by non-voluntary EMG signals (i.e., spasticity). This could be solved by using synergies between muscles [44] instead of experimentally recorded EMGs. With muscle synergies, muscle activations are represented by recombination of a small set of basic signals or primitives. These primitives are timed using the phase of the gait. One limitation with this solution is that the primitives are task-dependent and thus would reduce the usability of the system to only certain tasks. Even more problematic, when EMGs are recorded from stroke patients, spastic activities can appear. Part of them can be filtered out but a better solution would be to directly remove groups of unhealthy motor neurons from the signal. That could be done by using high density (HD-)EMG recording with real-time decomposition at the moto-neuron level [154] and to then removing (or filtering out) pathological moto-neuron. A notable benefit of this approach is that decomposition can record neural activities from SCI patients with complete lesion [155].

Another limitation of this method is its current dependency on the technical limitations of surface EMG. This can be seen in the difficulty to have the same level of EMG between recording sessions due to placement and replacement of EMG sensors, change in skin condition, movement artefacts and so one. This problem could also be solved by using textile HD-EMG with automatic muscle detection and real-time decomposition where the neural drive from the spinal cord could be used to drive our HMI [71].

We showed that we can assist users using wearable robotic devices by giving back a percentage of the users' joint torques. This can be useful for an industrial or military setting but this assistance has never been shown to deliver better outcomes over classic rehabilitations [83], e.g., stroke [84], [85], SCI patients [86]. Long term study on the effects of the assistance provided by our HMI for rehabilitations would be an interesting future work. Furthermore, our HMI can deliver information not only about joint mechanical properties but also about muscle mechanical properties. Putting this information into a rehabilitation controller with a clear goal (targeting at the muscle level) could also be beneficial for patients. Additionally, other joint mechanical variables such as joint stiffnesses [16] and joint compressive loads [15], could be added to the model and computed in realtime. Being able to reduce these parameters could have a clear impact on some groups of patients such as Duchenne patients or osteoarthritis patients. Due to the difficulty to obtain these parameters (i.e. stiffnesses and joint compressive loads) little research has been done to use these parameters in wearable robotic device controllers. Besides, by offering access to all of these internal parameters to clinicians, more informed decisions on which rehabilitation method to use can be made.

Finally, exoskeletons are still bulky with heavy components and low ergonomics which can be difficult for users, especially patients, to use for a long period. Soft exosuits [6] transfer some of the load of actuators from the user's limb to the user's back and are generally lighter. Soft exosuits still have issues with ergonomics as forces meant for only one joint can be 
distributed to the whole body. Currently, they can only offer low torque assistance when fully wearable due to friction and shear forces.

\subsection{Final Conclusion}

In this dissertation, an HMI was developed that, for the first time, could accurately compute multi-DOF joint torques from experimental EMGs and joint positions in real-time. This HMI was used to control lower-limb exoskeletons to assist healthy users during walking tasks, as well as paretic patients during seated tasks. Reductions of EMG amplitudes and joint torque levels were seen in both scenarios, which proved the positive effects of this new interface. We believe that this new tool can provide a higher quality of rehabilitation care for patients through wearable robotic devices. The next steps along realizing this include making the HMI easier to use by working on aspects such as EMG recording, creating a wearable robot's controller focused on personalized neurorehabilitation, and further validating the model estimated neuromuscular variables. 


\section{REFERENCES}

[1] H. Wang et al., "Global, regional, and national life expectancy, all-cause mortality, and cause-specific mortality for 249 causes of death, 1980-2015: a systematic analysis for the Global Burden of Disease Study 2015," Lancet, vol. 388, no. 10053, pp. 1459-1544, 2016.

[2] G. M. Rommers, L. D. W. Vos, J. W. Groothoff, C. H. Schuiling, and W. H. Eisma, "Epidemiology of lower limb amputees in the north of The Netherlands: aetiology, discharge destination and prosthetic use," Prosthet. Orthot. Int., vol. 21, no. 2, pp. 92 99, 1997.

[3] H. Burger, "Return to work after amputation," in Amputation, prosthesis use, and phantom limb pain, Springer, 2009, pp. 101-114.

[4] B. M. A. Huisstede, S. M. A. Bierma-Zeinstra, B. W. Koes, and J. A. N. Verhaar, "Incidence and prevalence of upper-extremity musculoskeletal disorders. A systematic appraisal of the literature," BMC Musculoskelet. Disord., vol. 7, no. 1, p. 7, 2006.

[5] D. Farina and O. Aszmann, "Bionic Limbs: Clinical Reality and Academic Promises.," Sci. Transl. Med., vol. 6, no. 257, p. 257ps12, Oct. 2014.

[6] F. A. Panizzolo et al., "A biologically-inspired multi-joint soft exosuit that can reduce the energy cost of loaded walking," J. Neuroeng. Rehabil., vol. 13, no. 1, p. 43, 2016.

[7] C. Meijneke, W. van Dijk, and H. van der Kooij, “Achilles: An autonomous lightweight ankle exoskeleton to provide push-off power," 5th IEEE RAS/EMBS Int. Conf. Biomed. Robot. Biomechatronics, pp. 918-923, 2014.

[8] B. G. Lapatki, J. P. Van Dijk, I. E. Jonas, M. J. Zwarts, and D. F. Stegeman, “A thin, flexible multielectrode grid for high-density surface EMG," J. Appl. Physiol., vol. 96, no. 1, pp. 327-36, 2004.

[9] T. A. Kuiken et al., "Targeted muscle reinnervation for real-time myoelectric control of multifunction artificial arms," Jama, vol. 301, no. 6, pp. 619-628, 2009.

[10] T. A. Kuiken, P. D. Marasco, B. A. Lock, R. N. Harden, and J. P. A. Dewald, "Redirection of cutaneous sensation from the hand to the chest skin of human amputees with targeted reinnervation," Proc. Natl. Acad. Sci., vol. 104, no. 50, pp. 20061-20066, 2007.

[11] H. Kazerooni, J.-L. J.-L. Racine, R. Steger, L. Huang, and R. Steger, "On the control of the berkeley lower extremity exoskeleton (BLEEX)," in Robotics and automation, 2005. ICRA 2005. Proceedings of the 2005 IEEE international conference on, 2005, no. April, pp. 4353-4360.

[12] C. G. A. Juanjuan Zhang, Pieter Fiers, Kirby A. Witte, Rachel W. Jackson, Katherine L. Poggensee and S. H. Collins, "Human-in-the-loop optimization of exoskeleton 
assistance during walking," Sci. Robot., 2017.

[13] N. Jiang et al., "Myoelectric Control of Artificial Limbs\&\#8212;Is There a Need to Change Focus? [In the Spotlight]," IEEE Signal Process. Mag., vol. 29, no. 5, pp. 152 150, 2012.

[14] M. Sartori, M. Reggiani, D. Farina, and D. G. Lloyd, "EMG-driven forward-dynamic estimation of muscle force and joint moment about multiple degrees of freedom in the human lower extremity.," PLoS One, vol. 7, no. 12, 2011.

[15] P. Gerus et al., "Subject-specific knee joint geometry improves predictions of medial tibiofemoral contact forces," J. Biomech., vol. 46, no. 16, pp. 2778-2786, 2013.

[16] M. Sartori et al., "Modeling and simulating the neuromuscular mechanisms regulating ankle and knee joint stiffness during human locomotion," J. Neurophysiol., vol. 114, no. 4, pp. 2509-2527, 2015.

[17] M. Sartori, D. G. Llyod, and D. Farina, "Neural Data-Driven Musculoskeletal Modeling for Personalized Neurorehabilitation Technologies," IEEE Trans. Biomed. Eng., vol. 63, no. 5, pp. 879-893, 2016.

[18] M. Sartori, G. Durandau, S. Došen, and D. Farina, "Robust simultaneous myoelectric control of multiple degrees of freedom in wrist-hand prostheses by realtime neuromusculoskeletal modeling," J. Neural Eng., vol. 15, no. 6, p. 66026, 2018.

[19] N. Lotti et al., "Adaptive Model-Based Myoelectric Control for a Soft Wearable Arm Exosuit: A New Generation of Wearable Robot Control," IEEE Robot. Autom. Mag., vol. 27, no. 1, pp. 43-53, Mar. 2020.

[20] A. V Hill, "The heat of shortening and the dynamic constants of muscle," Proc. R. Soc. London B Biol. Sci., vol. 126, no. 843, pp. 136-195, 1938.

[21] M. Sartori, M. Reggiani, A. J. van den Bogert, and D. G. Lloyd, "Estimation of musculotendon kinematics in large musculoskeletal models using multidimensional B-splines," J. Biomech., vol. 45, no. 3, pp. 595-601, 2012.

[22] D. G. Lloyd and T. F. Besier, "An EMG-driven musculoskeletal model to estimate muscle forces and knee joint moments in vivo," J. Biomech., vol. 36, no. 6, pp. 765776, 2003.

[23] E. E. Cavallaro, J. Rosen, J. C. Perry, and S. Burns, "Real-time myoprocessors for a neural controlled powered exoskeleton arm.," IEEE Trans. Biomed. Eng., vol. 53, no. 11, pp. 2387-2396, 2006.

[24] C. Fleischer and G. Hommel, "A human-exoskeleton interface utilizing electromyography," IEEE Trans. Robot., vol. 24, no. 4, pp. 872-882, 2008.

[25] S. L. Delp et al., "OpenSim: open-source software to create and analyze dynamic simulations of movement," Biomed. Eng. IEEE Trans., vol. 54, no. 11, pp. 1940-1950, 2007.

[26] M. E. Hahn, "Feasibility of estimating isokinetic knee torque using a neural network model," J. Biomech., vol. 40, no. 5, pp. 1107-1114, 2007. 
[27] K. Manal, R. V Gonzalez, D. G. Lloyd, and T. S. Buchanan, "A real-time EMGdriven virtual arm," Comput. Biol. Med., vol. 32, no. 1, pp. 25-36, 2002.

[28] K. Manal, K. Gravare-Silbernagel, and T. S. Buchanan, "A real-time EMG-driven musculoskeletal model of the ankle," Multibody Syst. Dyn., vol. 28, no. 1-2, pp. 169180, 2012.

[29] J. Kim et al., "Reducing the metabolic rate of walking and running with a versatile, portable exosuit," Science (80-. )., vol. 672, no. August, pp. 668-672, 2019.

[30] J. Zhang et al., "Human-in-the-loop optimization of exoskeleton assistance during walking," Science (80-. )., vol. 356, no. 6344, pp. 1280-1283, 2017.

[31] W. Van Dijk, C. Meijneke, H. Van Der Kooij, W. van Dijk, C. Meijneke, and H. van der Kooij, "Evaluation of the Achilles Ankle Exoskeleton.," IEEE Trans. Neural Syst. Rehabil. Eng., vol. 25, no. 2, pp. 151-160, 2017.

[32] M. Bortole et al., "The $\mathrm{H} 2$ robotic exoskeleton for gait rehabilitation after stroke: early findings from a clinical study," J. Neuroeng. Rehabil., vol. 12, no. 1, p. 54, 2015.

[33] C. Meijneke, S. Wang, V. Sluiter, and H. van der Kooij, "Introducing a modular, personalized exoskeleton for ankle and knee support of individuals with a spinal cord injury," in Wearable robotics: challenges and trends, Springer, 2017, pp. 169-173.

[34] M. G. Pandy, "Computer modeling and simulation of human movement," Annu. Rev. Biomed. Eng., vol. 3, no. 1, pp. 245-273, 2001.

[35] M. G. Hoy, F. E. Zajac, and M. E. Gordon, “A musculoskeletal model of the human lower extremity: the effect of muscle, tendon, and moment arm on the momentangle relationship of musculotendon actuators at the hip, knee, and ankle," $J$. Biomech., vol. 23, no. 2, pp. 157-169, 1990.

[36] S. S. M. Lee, A. S. Arnold, M. de Boef Miara, A. A. Biewener, and J. M. Wakeling, "Accuracy of gastrocnemius muscles forces in walking and running goats predicted by one-element and two-element Hill-type models," J. Biomech., vol. 46, no. 13, pp. 2288-2295, 2013.

[37] M. Damsgaard, J. Rasmussen, S. T. Christensen, E. Surma, and M. De Zee, “Analysis of musculoskeletal systems in the AnyBody Modeling System," Simul. Model. Pract. Theory, vol. 14, no. 8, pp. 1100-1111, 2006.

[38] A. J. den Bogert, T. Geijtenbeek, O. Even-Zohar, F. Steenbrink, and E. C. Hardin, "A real-time system for biomechanical analysis of human movement and muscle function," Med. Biol. Eng. Comput., vol. 51, no. 10, pp. 1069-1077, 2013.

[39] A. Murai, K. Kurosaki, K. Yamane, and Y. Nakamura, "Musculoskeletal-seethrough mirror: Computational modeling and algorithm for whole-body muscle activity visualization in real time," Prog. Biophys. Mol. Biol., vol. 103, no. 2, pp. 310 $317,2010$.

[40] R. D. Crowninshield and R. A. Brand, “A physiologically based criterion of muscle 
force prediction in locomotion,” J. Biomech., vol. 14, no. 11, pp. 793-801, 1981.

[41] W. Herzog and T. R. Leonard, "Validation of optimization models that estimate the forces exerted by synergistic muscles," J. Biomech., vol. 24, pp. 31-39, 1991.

[42] L. L. Menegaldo, L. F. de Oliveira, and K. K. Minato, "EMGD-FE: an open source graphical user interface for estimating isometric muscle forces in the lower limb using an EMG-driven model," Biomed. Eng. Online, vol. 13, no. 1, p. 1, 2014.

[43] Q. Shao, D. N. Bassett, K. Manal, and T. S. Buchanan, “An EMG-driven model to estimate muscle forces and joint moments in stroke patients," Comput. Biol. Med., vol. 39, no. 12, pp. 1083-1088, 2009.

[44] M. Sartori, L. Gizzi, D. G. D. G. Lloyd, and D. Farina, “A musculoskeletal model of human locomotion driven by a low dimensional set of impulsive excitation primitives," Front. Comput. Neurosci., vol. 7, no. June, pp. 1-22, 2013.

[45] A. L. Kinney, T. F. Besier, D. D. D’Lima, and B. J. Fregly, "Update on grand challenge competition to predict in vivo knee loads," J. Biomech. Eng., vol. 135, no. 2, p. 21012, 2013.

[46] M. Sartori, M. Maculan, C. Pizzolato, M. Reggiani, and D. Farina, "Modeling and simulating the neuromuscular mechanisms regulating ankle and knee joint stiffness during human locomotion," J. Neurophysiol., vol. 114, no. 4, pp. 2509-2527, 2015.

[47] E. J. Perreault, R. F. Kirsch, and P. E. Crago, "Voluntary control of static endpoint stiffness during force regulation tasks," J. Neurophysiol., vol. 87, no. 6, pp. 2808-2816, 2002.

[48] S. Heintz and E. M. Gutierrez-Farewik, "Static optimization of muscle forces during gait in comparison to EMG-to-force processing approach," Gait Posture, vol. 26, no. 2, pp. 279-288, 2007.

[49] J. P. Walter et al., "Muscle synergies may improve optimization prediction of knee contact forces during walking," J. Biomech. Eng., vol. 136, no. 2, p. 21031, 2014.

[50] E. K. Chadwick, D. Blana, A. J. van den Bogert, R. F. Kirsch, and others, "A realtime, 3-D musculoskeletal model for dynamic simulation of arm movements," IEEE Trans. Biomed. Eng., vol. 56, no. 4, pp. 941-948, 2009.

[51] C. Pizzolato et al., "CEINMS: A toolbox to investigate the influence of different neural control solutions on the prediction of muscle excitation and joint moments during dynamic motor tasks," J. Biomech., vol. 48, no. 14, pp. 3929-3936, 2015.

[52] S. L. Delp, J. P. Loan, M. G. Hoy, F. E. Zajac, E. L. Topp, and J. M. Rosen, “An interactive graphics-based model of the lower extremity to study orthopaedic surgical procedures," IEEE Trans. Biomed. Eng., vol. 37, no. 8, pp. 757-767, 1990.

[53] F. De Groote, T. De Laet, I. Jonkers, and J. De Schutter, "Kalman smoothing improves the estimation of joint kinematics and kinetics in marker-based human gait analysis," vol. 41, pp. 3390-3398, 2008.

[54] K. Manal and T. S. Buchanan, "A one-parameter neural activation to muscle 
activation model: estimating isometric joint moments from electromyograms," J. Biomech., vol. 36, no. 8, pp. 1197-1202, 2003.

[55] M. Sartori, M. Reggiani, E. Pagello, and D. G. Lloyd, "Modeling the human knee for assistive technologies," IEEE Trans. Biomed. Eng., vol. 59, no. 9, pp. 2642-2649, 2012.

[56] C. R. Winby, D. G. Lloyd, and T. B. Kirk, "Evaluation of different analytical methods for subject-specific scaling of musculotendon parameters," J. Biomech., vol. 41, no. 8, pp. 1682-1688, 2008.

[57] W. L. Goffe, G. D. Ferrier, and J. Rogers, "Global optimization of statistical functions with simulated annealing," J. Econom., vol. 60, no. 1-2, pp. 65-99, 1994.

[58] R. Grasso, L. Bianchi, and F. Lacquaniti, "Motor patterns for human gait: backward versus forward locomotion,” J. Neurophysiol., vol. 80, no. 4, pp. 1868-1885, 1998.

[59] S. A. Horan, S. L. Watson, C. P. Carty, M. Sartori, and B. K. Weeks, "Lower-limb kinematics of single-leg squat performance in young adults," Physiother. Canada, vol. 66, no. 3, pp. 228-233, 2014.

[60] M. Q. Liu, F. C. Anderson, M. H. Schwartz, and S. L. Delp, "Muscle contributions to support and progression over a range of walking speeds," J. Biomech., vol. 41, no. 15, pp. 3243-3252, 2008.

[61] M. Błażkiewicz, "Muscle force distribution during forward and backward locomotion," Acta Bioeng. Biomech., vol. 15, no. 3, pp. 3-9, 2013.

[62] J. R. Pope, "Comparison of Scaled vs. Ultrasound Based Musculoskeletal Models on Knee Muscle Moments During Single-Leg Squatting," 2012.

[63] R. Grasso, Y. P. Ivanenko, M. Zago, M. Molinari, G. Scivoletto, and F. Lacquaniti, "Recovery of forward stepping in spinal cord injured patients does not transfer to untrained backward stepping," Exp. brain Res., vol. 157, no. 3, pp. 377-382, 2004.

[64] R. W. M. van Deursen, T. W. Flynn, J. L. McCrory, and E. Morag, "Does a single control mechanism exist for both forward and backward walking?," Gait Posture, vol. 7, no. 3, pp. 214-224, 1998.

[65] T. S. Buchanan, D. G. Lloyd, K. Manal, T. F. Besier, and others, "Estimation of muscle forces and joint moments using a forward-inverse dynamics model," Med. Sci. Sports Exerc., vol. 37, no. 11, p. 1911, 2005.

[66] M. M. van der Krogt, L. Bar-On, T. Kindt, K. Desloovere, and J. Harlaar, "Neuromusculoskeletal simulation of instrumented contracture and spasticity assessment in children with cerebral palsy," J. Neuroeng. Rehabil., vol. 13, no. 1, p. 64, 2016.

[67] M. Sartori et al., "Toward modeling locomotion using electromyography-informed 3D models: application to cerebral palsy,” WIREs Syst. Biol. Med., p. e1368, 2017.

[68] P. Malcolm, W. Derave, S. Galle, and D. De Clercq, "A Simple Exoskeleton That Assists Plantarflexion Can Reduce the Metabolic Cost of Human Walking," PLoS 
One, vol. 8, no. 2, pp. 1-7, 2013.

[69] L. M. Mooney, E. J. Rouse, and H. M. Herr, "Autonomous exoskeleton reduces metabolic cost of human walking Autonomous exoskeleton reduces metabolic cost of human walking," vol. 11, no. 1, pp. 1-5, 2014.

[70] S. H. Collins, M. B. Wiggin, and G. S. Sawicki, "Reducing the energy cost of human walking using an unpowered exoskeleton," Nature, vol. 522, no. 7555, pp. 212-215, 2015.

[71] M. Sartori, U. S. Yavuz, and D. Farina, "In Vivo Neuromechanics: Decoding Causal Motor Neuron Behavior with Resulting Musculoskeletal Function," Sci. Rep., vol. 7, p. 13465, 2017.

[72] B. R. Griffin et al., "Stepping Forward with Exoskeletons," no. November, pp. 6674, 2017.

[73] J. Jang, K. Kim, J. Lee, B. Lim, and Y. Shim, "Online gait task recognition algorithm for hip exoskeleton,” IEEE Int. Conf. Intell. Robot. Syst., vol. 2015-Decem, pp. 53275332, 2015.

[74] A. Úbeda, J. M. Azorín, D. Farina, and M. Sartori, "Estimation of Neuromuscular Primitives from EEG Slow Cortical Potentials in Incomplete Spinal Cord Injury Individuals for a New Class of Brain-Machine Interfaces," Front. Comput. Neurosci., vol. 12, p. 3, Jan. 2018.

[75] J. F. Veneman, R. Kruidhof, E. E. G. Hekman, R. Ekkelenkamp, E. H. F. Van Asseldonk, and H. Van Der Kooij, "Design and evaluation of the LOPES exoskeleton robot for interactive gait rehabilitation," IEEE Trans. Neural Syst. Rehabil. Eng., vol. 15, no. 3, pp. 379-386, 2007.

[76] H. Kazerooni, J.-L. Racine, and R. Steger, "On the Control of the Berkeley Lower Extremity Exoskeleton (BLEEX)," Proc. 2005 IEEE Int. Conf. Robot. Autom., no. April, pp. 4353-4360, 2005.

[77] D. P. Ferris and C. L. Lewis, "Robotic Lower Limb Exoskeletons Using Proportional Myoelectric Control,” pp. 2119-2124, 2013.

[78] V. R. Garate, A. Parri, T. Yan, M. Munih, R. Molino, and R. Ronsse, "Experimental Validation of Motor Primitive-Based Control for Leg Exoskeletons during Continuous Multi-Locomotion Tasks," vol. 11, no. March, 2017.

[79] G. Durandau, D. Farina, and M. Sartori, "Robust Real-Time Musculoskeletal Modeling driven by Electromyograms," IEEE Trans. Biomed. Eng., 2017.

[80] M. Sartori, D. Farina, and D. G. D. G. Lloyd, "Hybrid neuromusculoskeletal modeling to best track joint moments using a balance between muscle excitations derived from electromyograms and optimization," J. Biomech., vol. 47, no. 15, pp. 3613-3621, Oct. 2014.

[81] Y. Ding et al., "Biomechanical and physiological evaluation of multi-joint assistance with soft exosuits," IEEE Trans. Neural Syst. Rehabil. Eng., vol. 25, no. 2, pp. 119- 
$130,2017$.

[82] B. R. Umberger, K. G. M. Gerritsen, and P. E. Martin, "A model of human muscle energy expenditure.," Comput. Methods Biomech. Biomed. Engin., vol. 6, no. 2, pp. 99 $111,2003$.

[83] D. Reinkensmeyer and V. Dietz, Neurorebabilitation technology: Second edition. 2016.

[84] T. G. Hornby, D. D. Campbell, J. H. Kahn, T. Demott, J. L. Moore, and H. R. Roth, "Enhanced gait-related improvements after therapist- versus robotic-assisted locomotor training in subjects with chronic stroke: A randomized controlled study,' Stroke, vol. 39, no. 6, pp. 1786-1792, 2008.

[85] J. Hidler et al., "Multicenter randomized clinical trial evaluating the effectiveness of the Lokomat in subacute stroke," Neurorehabil. Neural Repair, vol. 23, no. 1, pp. 5-13, 2009.

[86] E. Swinnen, S. Duerinck, J. Baeyens, R. Meeusen, and E. Kerckhofs, "Effectiveness of robot-assisted gait training in persons with spinal cord injury: A systematic review," J. Rehabil. Med., vol. 42, no. 6, pp. 520-526, 2010.

[87] M. A. Dimyan and L. G. Cohen, "Neuroplasticity in the context of motor rehabilitation after stroke Michael," vol. 7, no. 2, pp. 76-85, 2016.

[88] S. C. Cramer et al., "Harnessing neuroplasticity for clinical applications," Brain, vol. 134, no. 6. pp. 1591-1609, 2011.

[89] V. Dietz, "Proprioception and locomotor disorders," Nat. Rev. Neurosci., vol. 3, no. 10, pp. 781-790, Oct. 2002.

[90] V. Dietz and T. Sinkjaer, "Spastic movement disorder: impaired reflex function and altered muscle mechanics," Lancet Neurol., vol. 6, no. 8, pp. 725-733, 2007.

[91] A. Esquenazi, M. Talaty, A. Packel, and M. Saulino, "The ReWalk powered exoskeleton to restore ambulatory function to individuals with thoracic-level motorcomplete spinal cord injury," Am. J. Phys. Med. Rehabil., vol. 91, no. 11, pp. 911-921, 2012.

[92] S. Jezernik, G. Colombo, T. Keller, H. Frueh, and M. Morari, "Robotic orthosis lokomat: A rehabilitation and research tool," Neuromodulation Technol. neural interface, vol. 6, no. 2, pp. 108-115, 2003.

[93] K. P. Westlake and C. Patten, "Pilot study of Lokomat versus manual-assisted treadmill training for locomotor recovery post-stroke," J. Neuroeng. Rehabil., vol. 6 , no. 1, p. 18, 2009.

[94] T. Yan, M. Cempini, C. M. Oddo, and N. Vitiello, "Review of assistive strategies in powered lower-limb orthoses and exoskeletons," Rob. Auton. Syst., vol. 64, pp. 120 136, 2015.

[95] W. Huo, S. Mohammed, J. C. Moreno, and Y. Amirat, "Lower Limb Wearable Robots for Assistance and Rehabilitation: A State of the Art," IEEE Syst. J. 
[96] F. Attneave, M. B., and D. O. Hebb, "The Organization of Behavior; A Neuropsychological Theory," Am. J. Psychol., vol. 63, no. 4, p. 633, 1950.

[97] Z. F. Lerner, D. L. Damiano, and T. C. Bulea, "A lower-extremity exoskeleton improves knee extension in children with crouch gait from cerebral palsy," Sci. Transl. Med., vol. 9, no. 404, 2017.

[98] I. Cajigas, A. Koenig, G. Severini, M. Smith, and P. Bonato, "Robot-Induced Perturbations of Human Walking Reveal a Selective Generation of Motor Adaptation," Sci. Robot., vol. 2, no. 6, pp. 1-10, 2017.

[99] L. N. Awad et al., "A soft robotic exosuit improves walking in patients after stroke," Sci. Transl. Med., vol. 9, no. 400, 2017.

[100] J. Kang, D. Martelli, V. Vashista, I. Martinez-Hernandez, H. Kim, and S. K. Agrawal, "Robot-driven downward pelvic pull to improve crouch gait in children with cerebral palsy," Sci. Robot., vol. 2, no. 8, p. eaan2634, 2017.

[101] R. Huang, H. Cheng, Q. M. Chen, H. T. Tran, X. C. Lin, and Ieee, "Interactive Learning for Sensitivity Factors of a Human-Powered Augmentation Lower Exoskeleton," in 2015 Ieee/Rsj International Conference on Intelligent Robots and Systems, 2015, pp. 6409-6415.

[102] Z. Yang, W. Gu, J. Zhang, and L. Gui, "Sensitivity Amplification Control of Exoskeleton Suit," in Force Control Theory and Method of Human Load Carrying Exoskeleton Suit, Berlin, Heidelberg: Springer Berlin Heidelberg, 2017, pp. 73-101.

[103] H. Kawamoto and Y. Sankai, "Power assist method based on Phase Sequence and muscle force condition for HAL," Adv. Robot., vol. 19, no. 7, pp. 717-734, 2005.

[104] K. Kiguchi, T. Tanaka, and T. Fukuda, "Neuro-Fuzzy Control of a Robotic Exoskeleton With EMG Signals,” Informatica, vol. 12, no. 4, pp. 481-490, 2004.

[105] S. M. Cain, K. E. Gordon, and D. P. Ferris, "Locomotor adaptation to a powered ankle-foot orthosis depends on control method," J. Neuroeng. Rebabil., vol. 4, no. 1, p. 48, 2007.

[106] K. Z. Takahashi, M. D. Lewek, and G. S. Sawicki, "A neuromechanics-based powered ankle exoskeleton to assist walking post-stroke : a feasibility study," pp. 113, 2015.

[107] E. M. McCain et al., "Mechanics and energetics of post-stroke walking aided by a powered ankle exoskeleton with speed-adaptive myoelectric control," J. Neuroeng. Rehabil., vol. 16, no. 1, pp. 1-12, 2019.

[108] A. Tang and W. Z. Rymer, "Abnormal force--EMG relations in paretic limbs of hemiparetic human subjects.," J. Neurol. Neurosurg. Psychiatry, vol. 44, no. 8, pp. 690 8, 1981.

[109] P. Zhou, N. L. Suresh, and W. Z. Rymer, "Model based sensitivity analysis of EMGforce relation with respect to motor unit properties: Applications to muscle paresis in stroke," Ann. Biomed. Eng., vol. 35, no. 9, pp. 1521-1531, 2007. 
[110] K. Kiguchi, Y. Imada, and M. Liyanage, "EMG-based neuro-fuzzy control of a 4DOF upper-limb power-assist exoskeleton," in Annual International Conference of the IEEE Engineering in Medicine and Biology - Proceedings, 2007, pp. 3040-3043.

[111] E. Martinez Valdes, F. Negro, D. Falla, A. M. De Nunzio, and D. Farina, "Surface EMG amplitude does not identify differences in neural drive to synergistic muscles," J. Appl. Physiol., vol. 16, p. japplphysiol.01115.2017, Feb. 2018.

[112] M. Jochumsen, I. K. Niazi, N. Mrachacz-Kersting, D. Farina, and K. Dremstrup, "Detection and classification of movement-related cortical potentials associated with task force and speed.," J. Neural Eng., vol. 10, no. 5, p. 056015, 2013.

[113] T. Noda, N. Sugimoto, J. Furukawa, M. A. Sato, S. H. Hyon, and J. Morimoto, "Brain-controlled exoskeleton robot for BMI rehabilitation," IEEE-RAS Int. Conf. Humanoid Robot., pp. 21-27, 2012.

[114] N.-S. Kwak, K.-R. Müller, and S.-W. Lee, “A lower limb exoskeleton control system based on steady state visual evoked potentials," J. Neural Eng., vol. 12, no. 5, p. 056009, 2015.

[115] F. Zhu, M. Bortole, A. Venkatakrishnan, Hernandez, G. Francisco, and J. Pons, "Human-machine system for the H2 lower limb exoskeleton: Neural decoding of robot-assisted walking from scalp EEG for stroke rehabilitation," Neuromodulation, vol. 17, no. 5, p. e75, Jul. 2014.

[116] M. Sartori, D. Farina, and D. G. Lloyd, "Hybrid neuromusculoskeletal modeling to best track joint moments using a balance between muscle excitations derived from electromyograms and optimization," J. Biomech., vol. 47, no. 15, 2014.

[117] C. R. Winby, D. G. Lloyd, T. F. Besier, and T. B. Kirk, "Muscle and external load contribution to knee joint contact loads during normal gait.," J. Biomech., vol. 42, no. 14, pp. 2294-300, Oct. 2009.

[118] J. Adamson, A. Beswick, and S. Ebrahim, "Is stroke the most common cause of disability?,” J. Stroke Cerebrovasc. Dis., vol. 13, no. 4, pp. 171-177, 2004.

[119] T. E. Oliphant, "Python for scientific computing," Comput. Sci. Eng., vol. 9, no. 3, 2007.

[120] A. Nordez, T. Gallot, S. Catheline, A. Guével, C. Cornu, and F. Hug, "Electromechanical delay revisited using very high frame rate ultrasound.," J. Appl. Physiol., vol. 106, no. 6, pp. 1970-5, Jun. 2009.

[121] D. Farina and M. Sartori, "Surface Electromyography for Man-Machine Interfacing in Rehabilitation Technologies," in Surface Electromyography: Physiology, Engineering and Applications, 2nd ed., D. Farina and R. Merletti, Eds. IEEE/Wiley, 2016, pp. 540560.

[122] P. Parker, K. Englehart, and B. Hudgins, "Myoelectric signal processing for control of powered limb prostheses," J. Electromyogr. Kinesiol., vol. 16, pp. 541-548, 2006. 
[123] W. Z. Shuo-Hsiu Chang, Gerard E. Francisco, Ping Zhou and and S. L. Rymer, "Spasticity, weakness, force variability and sustained spontaneous motor unit discharges of resting spastic-paretic biceps brachii muscles in chronic stroke," vol. 48, no. 1, pp. 85-92, 2013.

[124] B. T. Volpe et al., "Intensive Sensorimotor Arm Training Mediated by Therapist or Robot Improves Hemiparesis in Patients With Chronic Stroke," Neurorebabil. Neural Repair, vol. 22, no. 3, pp. 305-310, 2008.

[125] J. W. Cohen, T. D. Ivanova, B. Brouwer, K. J. Miller, D. Bryant, and S. J. Garland, "Do Performance Measures of Strength, Balance, and Mobility Predict Quality of Life and Community Reintegration After Stroke?," Arch. Phys. Med. Rehabil., vol. 99, no. 4, pp. 713-719, 2018.

[126] I. L. Katzan, N. R. Thompson, K. Uchino, and B. Lapin, "The most affected health domains after ischemic stroke,” Neurology, vol. 90, no. 16, pp. e1364--e1371, 2018.

[127] N. Mahendran, S. S. Kuys, and S. G. Brauer, "Recovery of ambulation activity across the first six months post-stroke," Gait Posture, vol. 49, pp. 271-276, 2016.

[128] J. Mehrholz, S. Thomas, C. Werner, J. Kugler, M. Pohl, and B. Elsner, "Electromechanical-assisted training for walking after stroke," Cochrane Database Syst. Rev., no. 5, 2017.

[129] A. Ajoudani et al., "Smart Collaborative Systems for Enabling Flexible and Ergonomic Work Practices,” 2020.

[130] A. T. Asbeck, S. M. M. De Rossi, K. G. Holt, and C. J. Walsh, "A biologically inspired soft exosuit for walking assistance," Int. J. Rob. Res., vol. 34, no. 6, pp. 744 762, 2015.

[131] S. Galle, P. Malcolm, S. H. Collins, and D. De Clercq, "Reducing the metabolic cost of walking with an ankle exoskeleton: interaction between actuation timing and power," J. Neuroeng. Rehabil., vol. 14, no. 1, pp. 1-16, 2017.

[132] Y. Ding, M. Kim, S. Kuindersma, and C. J. Walsh, "Human-in-the-loop optimization of hip assistance with a soft exosuit during walking," Sci. Robot., vol. 3, no. 15, p. eaar5438, Feb. 2018.

[133] C. Khazoom, C. Veronneau, J. P. L. Bigue, J. Grenier, A. Girard, and J. S. Plante, "Design and control of a multifunctional ankle exoskeleton powered by magnetorheological actuators to assist walking, jumping, and landing," IEEE Robot. Autom. Lett., vol. 4, no. 3, pp. 3083-3090, 2019.

[134] R. W. Jackson and S. H. Collins, "Heuristic-Based Ankle Exoskeleton Control for Co-Adaptive Assistance of Human locomotion," IEEE Trans. Neural Syst. Rehabil. Eng., vol. PP, no. c, pp. 1-10, 2019.

[135] L. S. et al., "Autonomous multi-joint soft exosuit with augmentation-power-based control parameter tuning reduces energy cost of loaded walking," J. Neuroeng. Rebabil., vol. 15, no. 1, pp. 1-9, 2018. 
[136] J. R. Koller, C. D. Remy, and D. P. Ferris, "Biomechanics and energetics of walking in powered ankle exoskeletons using myoelectric control versus mechanically intrinsic control," J. Neuroeng. Rebabil., vol. 15, no. 1, pp. 1-14, 2018.

[137] G. S. Sawicki, O. N. Beck, I. Kang, and A. J. Young, "The exoskeleton expansion: Improving walking and running economy," J. Neuroeng. Rehabil., vol. 17, no. 1, pp. $1-9,2020$.

[138] B. Lim, J. Jang, J. Lee, B. Choi, Y. Lee, and Y. Shim, "Delayed Output Feedback Control for Gait Assistance and Resistance Using a Robotic Exoskeleton," IEEE Robot. Autom. Lett., vol. 4, no. 4, pp. 3521-3528, 2019.

[139] R. P. Brent, "Some efficient algorithms for solving systems of nonlinear equations," SLAM J. Numer. Anal., vol. 10, no. 2, pp. 327-344, 1973.

[140] A. Wächter and L. T. Biegler, "On the implementation of an interior-point filter linesearch algorithm for large-scale nonlinear programming," Math. Program., vol. 106, no. 1 , pp. $25-57,2006$.

[141] H. van der K. W.F. Rampeltshammer, A.Q.L. Keemink, "An improved force controller with low and passive apparent impedance for series elastic actuators," IEEE/ASME Trans. on Mechatronics.

[142] G. S. Sawicki and D. P. Ferris, "Mechanics and energetics of level walking with powered ankle exoskeletons," J. Exp. Biol., vol. 211, no. 9, pp. 1402-1413, 2008.

[143] N. Lotti et al., "Adaptive model-based myoelectric control for a soft wearable arm exosuit: A new generation of wearable robot control," IEEE Robot. Autom. Mag., p. $0,2020$.

[144] G. Durandau et al., "Voluntary control of wearable robotic exoskeletons by patients with paresis via neuromechanical modeling," J. Neuroeng. Rehabil., vol. 16, no. 1, p. $91,2019$.

[145] J. M. Caputo and S. H. Collins, "A universal ankle--foot prosthesis emulator for human locomotion experiments," J. Biomech. Eng., vol. 136, no. 3, 2014.

[146] C. P. Cop, G. Durandau, A. M. Esteban, R. C. van't Veld, A. C. Schouten, and M. Sartori, "Model-based estimation of ankle joint stiffness during dynamic tasks: a validation-based approach," in 201941 st Annual International Conference of the IEEE Engineering in Medicine and Biology Society (EMBC), 2019, pp. 4104-4107.

[147] C. Chen, S. Ma, X. Sheng, D. Farina, and X. Zhu, “Adaptive real-time identification of motor unit discharges from non-stationary high-density surface electromyographic signals," IEEE Trans. Biomed. Eng., 2020.

[148] R. M. Alexander, "The dimensions of knee and ankle muscles and the forces they exert," J. Hum. Move. Stud., vol. 1, pp. 115-123, 1975.

[149] T. Fukunaga et al., "Physiological cross-sectional area of human leg muscles based on magnetic resonance imaging," J. Orthop. Res., vol. 10, no. 6, pp. 926-934, 1992. 
[150] J. Fernandez et al., "Multiscale musculoskeletal modelling, data--model fusion and electromyography-informed modelling," Interface Focus, vol. 6, no. 2, p. 20150084, 2016.

[151] J. Zhang, J. Hislop-Jambrich, and T. F. Besier, "Predictive statistical models of baseline variations in 3-D femoral cortex morphology," Med. Eng. Phys., vol. 38, no. 5, pp. 450-457, 2016.

[152] P. Roriz, L. Carvalho, O. Frazão, J. L. Santos, and J. A. Simões, "From conventional sensors to fibre optic sensors for strain and force measurements in biomechanics applications: A review," J. Biomech., vol. 47, no. 6, pp. 1251-1261, 2014.

[153] J. A. Martin et al., "Gauging force by tapping tendons," Nat. Commun., vol. 9, no. 1, p. 1592, 2018.

[154] V. Glaser, A. Holobar, and D. Zazula, "Real-time motor unit identification from high-density surface EMG," IEEE Trans. neural Syst. Rehabil. Eng., vol. 21, no. 6, pp. 949-958, 2013.

[155] J. Ting et al., "A wearable neural interface for detecting and decoding attempted hand movements in a person with tetraplegia," in 2019 41st Annual International Conference of the IEEE Engineering in Medicine and Biology Society (EMBC), 2019, pp. 1930-1933.

[156] D. Farina, K. Yoshida, T. Stieglitz, and K. P. Koch, "Multichannel thin-film electrode for intramuscular electromyographic recordings," J. Appl. Physiol., vol. 104, no. 3, pp. 821-827, 2008.

[157] D. Farina et al., "Man/machine interface based on the discharge timings of spinal motor neurons after targeted muscle reinnervation," Nat. Biomed. Eng., vol. 1, no. 2, pp. 1-12, Feb. 2017.

[158] R. Merletti, A. Holobar, and D. Farina, "Analysis of motor units with high-density surface electromyography,” J. Electromyogr. Kinesiol., vol. 18, no. 6, pp. 879-890, 2008.

[159] D. G. Thelen and others, "Adjustment of muscle mechanics model parameters to simulate dynamic contractions in older adults," Trans. Soc. Mech. Eng. J. Biomech. Eng., vol. 125, no. 1, pp. 70-77, 2003.

[160] F. E. Zajac, "Muscle and tendon: properties, models, scaling, and application to biomechanics and motor control.," Critical reviews in biomedical engineering. 1989.

[161] M. A. Sherman, A. Seth, and S. L. Delp, "What is a moment arm? Calculating muscle effectiveness in biomechanical models using generalized coordinates," Proc. ASME Des. Eng. Tech. Conf., vol. 7 B, pp. 1-9, 2013. 


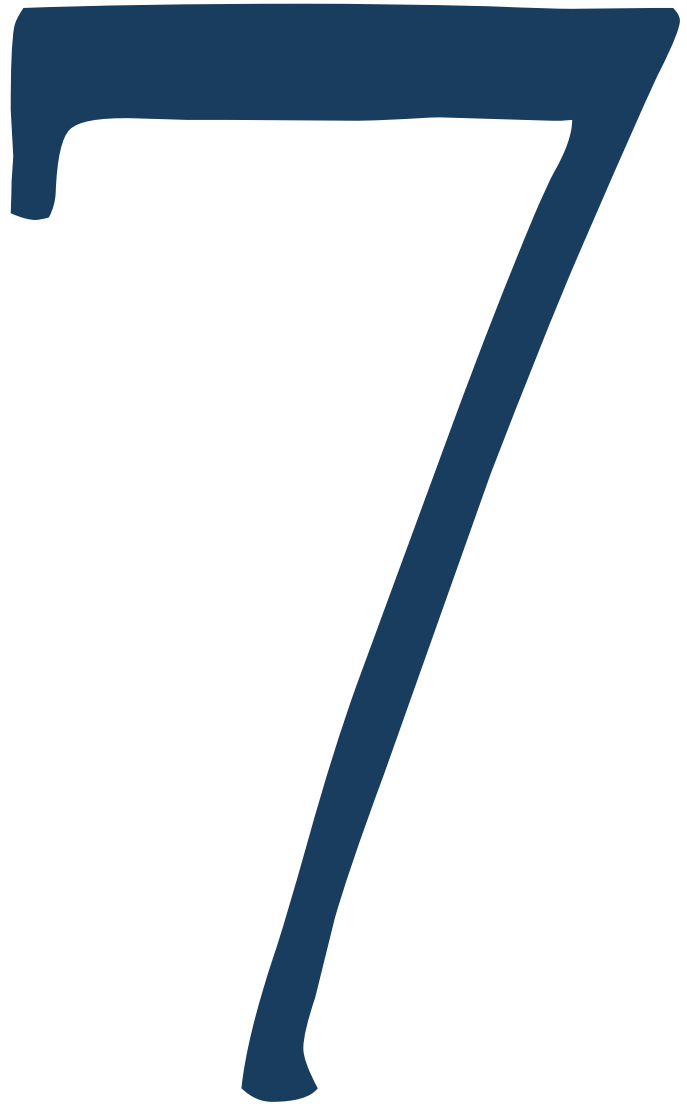




\subsection{Appendix to Introduction}

\subsubsection{Wearable Robotics}

\subsubsection{The different type of Wearable Robot}

In this thesis, we were interested in mainly two types of wearable robots: exoskeletons and prosthesis. An exoskeleton is a robotic device placed in parallel to the musculoskeletal system of the user and provides assistance to the joint. Exoskeletons can be rigid (i.e. all actuators are rigidly connected to the user and in-between them) or soft (i.e. soft interfaces are used with the user or in-between actuator). A prosthesis is placed on series to the musculoskeletal system of the user to replace a missing limb most of the time. The following devices were used during this PhD's thesis:

\subsection{Acbilles}

The Achilles exoskeleton is an autonomous rigid ankle exoskeleton [31] (Fig. 7-1-1) consisting of one backpack with computer and battery and two ankle modules (right and left). The ankle module consists of a series-elastic actuator (SEA) composed of a leaf spring, motor and spindle. The ankle modules also possess diverse sensors like joint encoder, motor
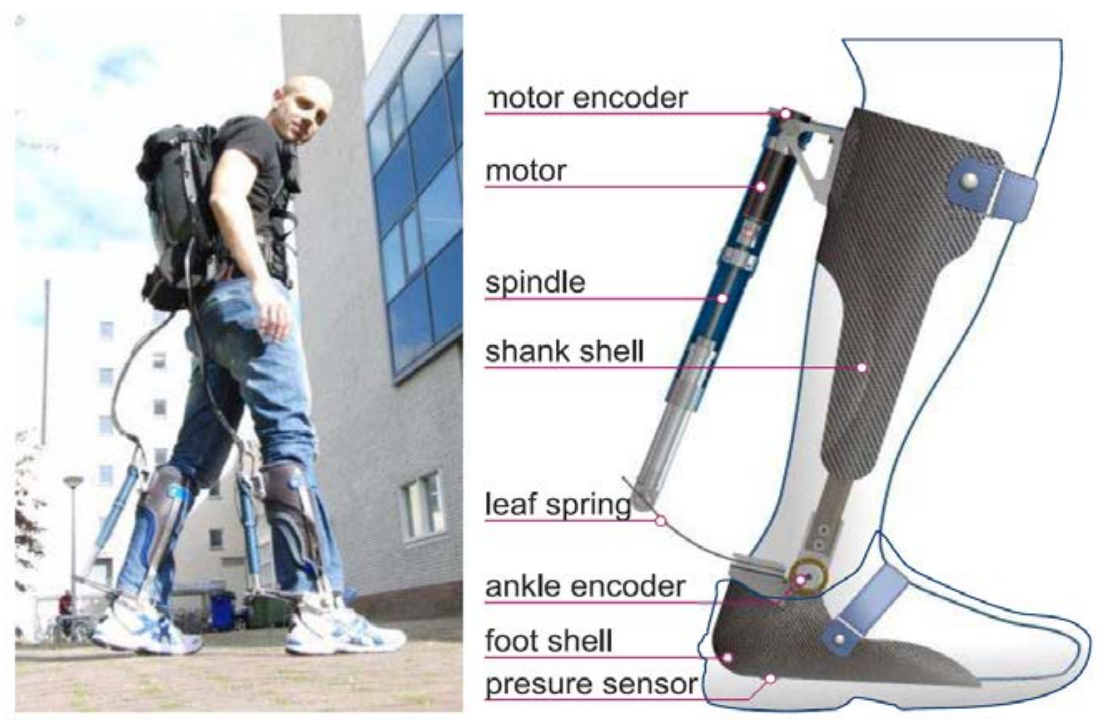

Figure 7-1-1: Achilles ankle exoskeleton (Image from [31]). 


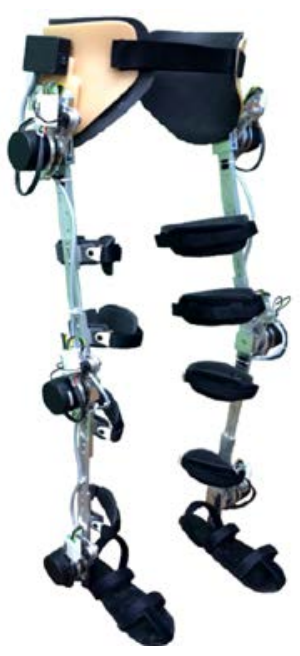

Figure 7-1-2: H2 exoskeleton (Image from [32]).

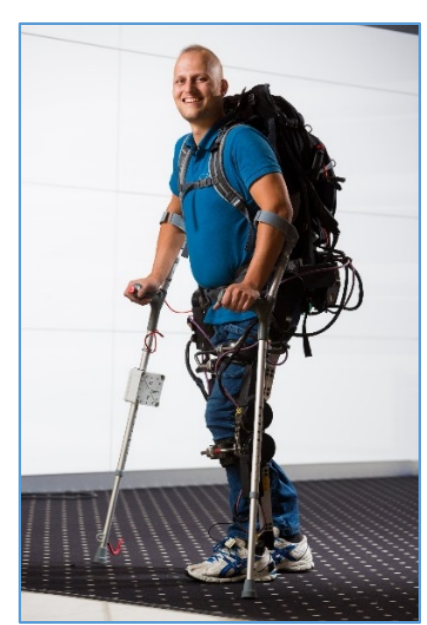

Figure 7-1-3: the WR2 exoskeleton.

encoder and pressure sensor. Communication between ankle modules and backpack is done via the real-time Ethercat network. The Achilles was developed by the group of Herman van der Kooij at the University of Twente and the University of Delft, the Netherlands. This exoskeleton was used for conducting experiments in Chapter 2.

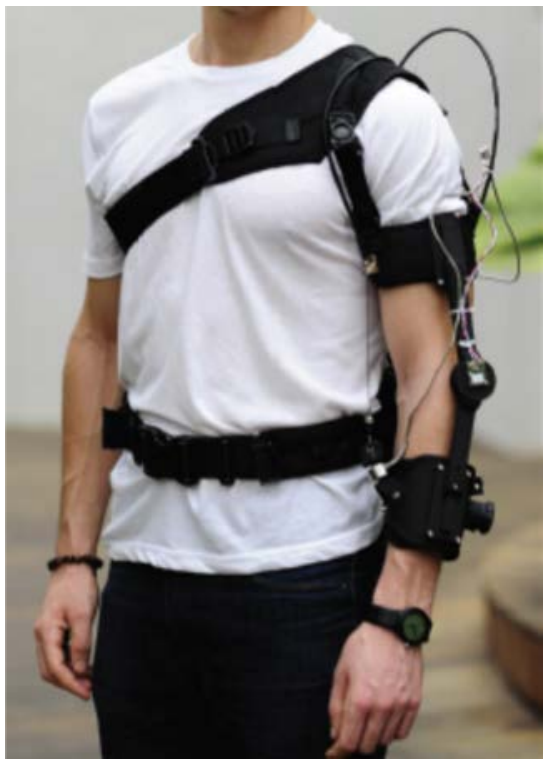

Figure 7-1-4: soft exosuit for the elbow (Image from [143]).

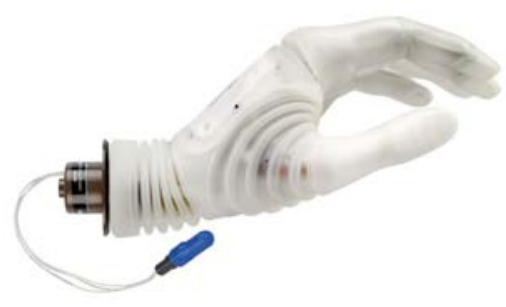

Figure 7-1-5: Michelangelo hand prosthesis from OttoBock (Image from ottobockus.com) 
7.1.1.1.2 H2

The $\mathrm{H} 2$ exoskeleton is a rigid lower-limb exoskeleton composed of 6 degrees of freedom (DOFs) (hip flexion-extension, knee flexion-extension and ankle dorsi-plantarflexion) [32] (Fig. 7-1-2). Each joint module has a motor with harmonic drive, motor sensor, position sensor, sole sensor and strain gauge. The $\mathrm{H} 2$ uses the real-time CAN network for communicating between modules. This exoskeleton was developed by the group of Jose L. Pons at the CSIC, Spain. The H2 was used for realizing experiments in Chapter 3.

\subsection{WR2}

The WR2 exoskeleton [33] (Fig. 7-1-3) was developed for the Symbitron European project lead by Herman van der Kooij. The WR2 has 12 DOFS (ten actives and two passives) and is a rigid exoskeleton. Each joint has a SEA with a motor, spring and harmonic drive. The exoskeleton also has a position sensor, a motor sensor for each active joint and sole switch sensor for each foot. Communication between DOF module and backpack is done via the EtherCAT network. This exoskeleton was used for conducting experiments in Chapter 4.

\subsection{Soft Exosuit}

The soft exosuit (Fig. 7-1-4) used during this $\mathrm{PhD}$, is a cable-driven exoskeleton for the elbow. It was developed by the group of Lorenzo Masia at Nanyang Technological University, Singapore. It is composed of a motor mounted on the upper arm driving a cable pulling the forearm. It also has the following sensors: motor encoder, load cell and position encoder at the elbow. This exoskeleton was used in [143].

\subsection{Michelangelo Hand}

The Michelangelo is a hand prosthesis with 3 DOFs (Hand open and close, wrist flexionextension and wrist rotation) (Fig. 7-1-5). This prosthesis is a commercial device developed by OttoBock, Germany. Connection to the device is done via Bluetooth. This device was used in [18].

\subsubsection{Torque Control of Wearable Robot}

The most common approach for the control of robotic devices in torque is a proportional derivative integrator (PID) controller, which tries to reduce the error between the torque command and experimental torque using the following equation:

$$
\omega=P . e(t)+D(e(t)-e(t-1))+I\left(e(t)+0.1 \sum_{0}^{t-1} e\right)
$$

With $\omega$ the velocity command for the motor, $\mathrm{P}$ the proportional gain, $e(t)$ error in torque between the command and the experimental torque at instant t, D the derivative gain and I the integrator gain.

This approach is used for the control of the $\mathrm{H} 2$ exoskeleton and the soft exosuit. 
For the Achilles exoskeleton, the torque control is done by controlling the spring deflection. A curve, calibrated beforehand is used to compute the desired spring deflection for the desired torque then a PD controller computes the desired motor command to reduce the error between spring deflection command and actual spring deflection. This can be summarized with the following equation:

$$
\omega=P . e(t)+D(e(t)-e(t-1))
$$

With $\omega$ the velocity command for the motor, $\mathrm{P}$ the proportional gain, $e(t)$ error at instant $\mathrm{t}$ and $\mathrm{D}$ derivative gain. $e(t)$ can be computed with the following equation:

$$
e(t)=f\left(\tau, \theta_{\text {exp }}\right)-p_{\text {exp }}
$$

eq. 1.6

With $f\left(\tau, \theta_{\text {exp }}\right)$ the torque spring deflection relationship, $\tau$ the torque command, $\theta_{\text {exp }}$ the experimental joint position and $p_{\text {exp }}$ the current spring deflection.

Finally, the latest development in torque control of exoskeleton can be found in the work of Rampeltshammer et al [141]. This controller was implemented in the WR2 exoskeleton. This controller is based on disturbance observer (DOB) and is composed of four elements, a PD controller, feedforward term, DOB and a model of the SEA actuator. More information can be found in [141].

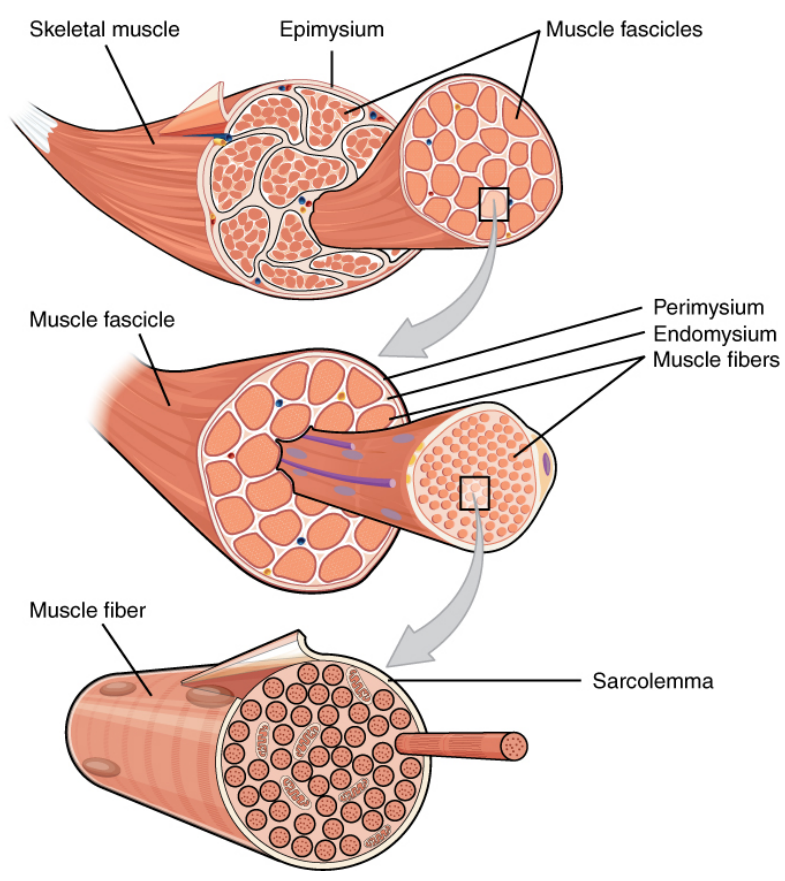

Figure 7-1-6: Muscle to fascicle to fiber (image from openstax.org). 


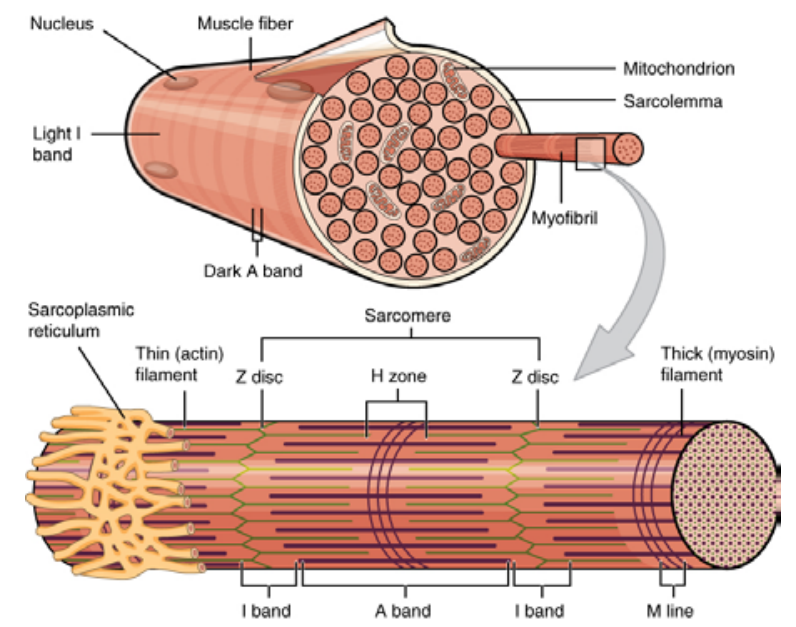

Figure 7-1-7: Muscle fiber to myofibrils (image from openstax.org).

\subsubsection{Neurophysiology of the Muscle}

\subsubsection{Muscle-Tendon unit physiology}

Muscle-tendon units (MTU) are the main force generator in our body. It is composed of a tendon that is attached to the skeletal system on one side and the muscle on the other side. Tendons are mainly made of collagen and act as a spring in the muscle-tendon system. Muscle, on the contrary, can actively produce force by twitching under the effect of motor unit action potential (MUAP) that is the main command signal of the muscle-tendon system. The muscle is mainly comprised of muscle fiber bundled together in fascicle (Fig. 7-1-6), which are activated together by the same MUAP.

Each muscle fibers are also known as muscle cells are composed of myofibrils (Fig. 7-1-7). Myofibrils are built of proteins (actin, myosin and titin) that are organized into myofilaments (Fig 7-1-8) that can slide along each other under the effect of MUAP creating muscle contraction.

For the central nervous system (CNS) to be aware of the MTU state, the MTU is composed of multiple proprioception mechanisms. For example, the muscle spindle type II will activate (or fire) depending on the stretch level of the muscle fiber giving information about the length of the muscle. Muscle spindle type I will activate depending on the rate of change in contraction giving information about the muscle velocity. Golgi tendon organ (GTO) named after Italian physician Camillo Golgi, is situated as the intersection between the muscle and the tendon. The GTO will activate when compressed by the muscle fiber thus encoding muscle force. 


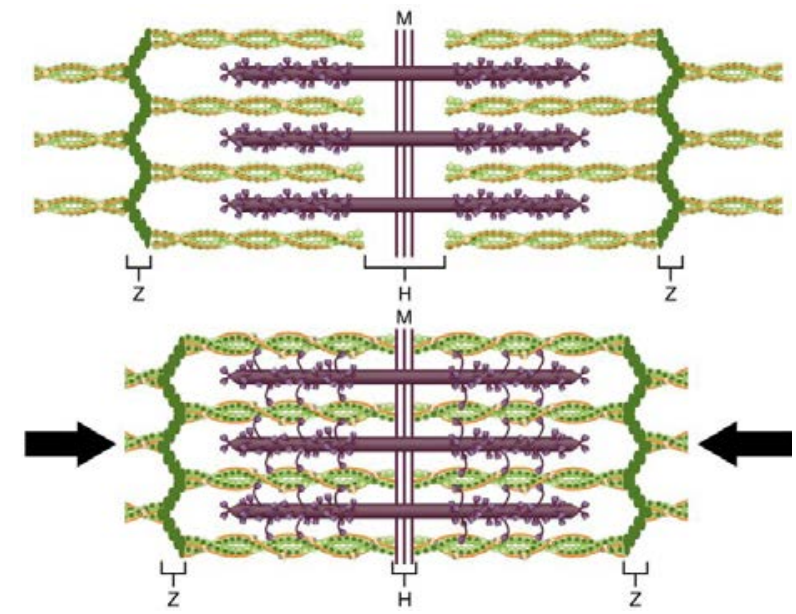

Figure 7-1-8: Myofilaments sliding to create force (image from openstax.org).

\subsubsection{Electromyography}

Electromyography (EMG) is a technique to record the electrical signal of the muscles. EMG represents the summation of all MUAP. It can be recorded using surface electrodes placed directly on the skin or via intramuscular electrodes using needles electrodes introduced in the muscle [156]. Surface electrodes are currently the most popular form of recording for EMG as other methods like needle electrodes are invasive method. Processing of EMG for use in neuromusculoskeletal modelling use the following methods:

1. High pass filtering;

2. Normalization;

3. Low pass filtering;

4. Normalization against maximal voluntary contraction.

A neuromusculoskeletal model can also directly be driven by MUAP from high density (HD) EMG recording [157]. HD EMG recording uses multiple electrodes in a matrix configuration to record EMG on the whole muscle. A decomposition algorithm can then be used to go back to the MUAP [158].

Information about surface electrode placement, shape, size and so on can be found on the SENIAM project website ${ }^{17}$.

\footnotetext{
${ }^{17}$ seniam.org/
} 


\subsubsection{Neuromusculoskeletal Modelling}

\subsubsection{Hill-type muscle model}

Hill's muscle model is a numerical representation of the muscle composed of three elements. It was first formulated by English physiologist Archibald Vivian Hill [20], [22], [159], [160] for which he received the 1922 Nobel prize in Physiology or Medicine.

The model is comprised of a contractile element (CE), parallel element (PE) and a series element (SE) (see Fig 7-1-9).

The muscle-tendon unit (MTU) can be approximate by the following equation:

$$
F^{M T U}(t)=F^{T}=F^{M a x}\left(f\left(L^{M}\right) f\left(V^{M}\right) E(t)+f_{p}\left(L^{M}\right)\right) \cos \left(\alpha^{M}(t)\right) \quad \text { eq. } 1.1
$$

With $F^{M T U}(t)$ the MTU force at instant $t, F^{T}$ the tendon force (SE), $F^{M a x}$ the maximum isometric force, $f\left(L^{M}\right)$ the force-length relationship (Fig 7-1-10), $f\left(V^{M}\right)$ the force-velocity relationship, $E(t)$ the normalized muscle activation at instant t. These values represent the

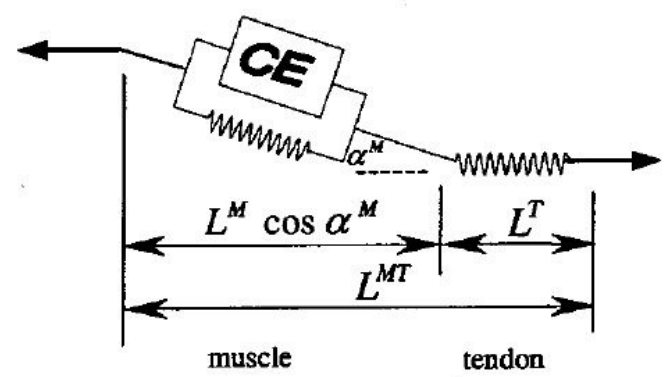

Figure 7-1-9: Hill's muscle model (Image from [159])

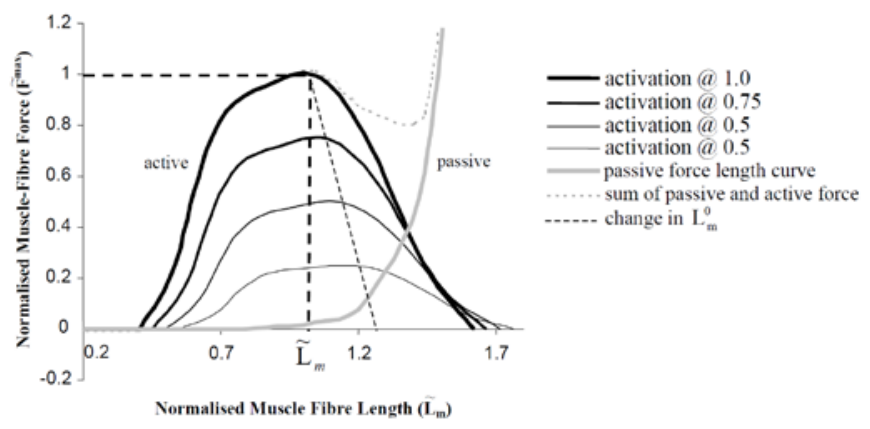

Figure 7-1-10: Active $f\left(L^{M}\right)$ and passive $f_{p}\left(L^{M}\right)$ force length curve (image from [22]). 
contractile elements (CE). We also have $f_{p}\left(L^{M}\right)$ the passive force-length relationship (PE) (Fig 1-2) and $\alpha^{M}(t)$ the pennation angle.

\subsubsection{From Muscles Force to Joints Torque}

Once all muscles' force acting on the joint of interest have been computed, these forces have to be projected on the joint using the moment arm (Fig 7-1-11). The moment arm is often computed numerically using the 'tendon excursion method' [161] using the following equation:

$$
r_{\theta}=\frac{d l}{d \theta}
$$

With $r_{\theta}$ the moment arm, $d l$ the MTU length displacement for $d \theta$ the displacement of the joint.

Finally, the joint torques are computed using the following formula:

$$
\tau=\sum_{m} r_{\theta, m} F_{m}^{M T U}
$$

With $\tau$ the joint torque, $r_{\theta, m}$ the moment arm for muscle $\mathrm{m}$ and $F_{m}^{M T U}$ the muscle force for muscle $\mathrm{m}$.

More information about the muscle model and joint torque computation used in the developed HMI can be found in chapter 2 .

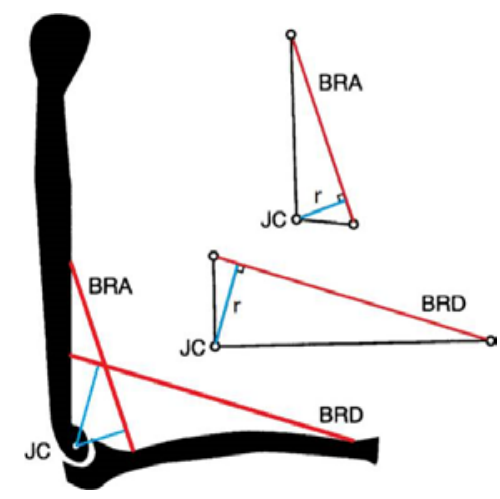

Figure 7-1-11: Moment arm computation for the elbow joint (Image from [161]). 


\subsection{Appendix to Chapter 2}

\subsubsection{Supplementary Tables}

Table 7-2-1:root mean squared error, standard deviation and Pearson correlation coefficients.

\begin{tabular}{|c|c|c|c|}
\hline Trial & DOF & $\begin{array}{c}\text { Mean Error }( \pm s t d) \\
(\mathrm{N} \cdot \mathrm{m} / \mathrm{Kg})\end{array}$ & $r( \pm s t d)$ \\
\hline \multirow{3}{*}{ Gait } & Knee & $0.13 \pm 0.11$ & $0.43 \pm 0.36$ \\
\hline & Ankle & $0.18 \pm 0.17$ & $0.90 \pm 0.07$ \\
\hline & Subtalar & $0.04 \pm 0.04$ & $0.85 \pm 0.24$ \\
\hline \multirow{3}{*}{ Squat } & Knee & $0.07 \pm 0.07$ & $0.88 \pm 0.10$ \\
\hline & Ankle & $0.10 \pm 0.07$ & $0.67 \pm 0.49$ \\
\hline & Subtalar & $0.07 \pm 0.02$ & $0.60 \pm 0.60$ \\
\hline \multirow{3}{*}{ Calf Rise } & Knee & $0.03 \pm 0.03$ & $0.77 \pm 0.19$ \\
\hline & Ankle & $0.13 \pm 0.09$ & $0.84 \pm 0.08$ \\
\hline & Subtalar & $0.05 \pm 0.03$ & $0.65 \pm 0.15$ \\
\hline \multirow{3}{*}{ Single Leg Squat } & Knee & $0.34 \pm 0.21$ & $0.89 \pm 0.08$ \\
\hline & Ankle & $0.25 \pm 0.14$ & $0.48 \pm 0.48$ \\
\hline & Subtalar & $0.01 \pm 0.01$ & $0.46 \pm 0.44$ \\
\hline \multirow{3}{*}{ Single Leg Calf Rise } & Knee & $0.37 \pm 0.12$ & $0.55 \pm 0.27$ \\
\hline & Ankle & $0.15 \pm 0.10$ & $0.86 \pm 0.05$ \\
\hline & Subtalar & $0.06 \pm 0.03$ & $0.63 \pm 0.21$ \\
\hline \multirow{3}{*}{ Fast Gait } & Knee & $0.20 \pm 0.15$ & $0.48 \pm 0.45$ \\
\hline & Ankle & $0.24 \pm 0.23$ & $0.74 \pm 0.35$ \\
\hline & Subtalar & $0.07 \pm 0.07$ & $0.67 \pm 0.33$ \\
\hline \multirow{3}{*}{ Backward Gait } & Knee & $0.12 \pm 0.12$ & $0.66 \pm 0.27$ \\
\hline & Ankle & $0.18 \pm 0.17$ & $0.78 \pm 0.17$ \\
\hline & Subtalar & $0.05 \pm 0.03$ & $0.66 \pm 0.25$ \\
\hline \multirow{3}{*}{ Jump } & Knee & $0.16 \pm 0.12$ & $0.58 \pm 0.22$ \\
\hline & Ankle & $0.17 \pm 0.16$ & $0.64 \pm 0.22$ \\
\hline & Subtalar & $0.05 \pm 0.04$ & $0.48 \pm 0.38$ \\
\hline \multirow{3}{*}{ Side Step } & Knee & $0.12 \pm 0.12$ & $0.68 \pm 0.27$ \\
\hline & Ankle & $0.18 \pm 0.14$ & $0.82 \pm 0.12$ \\
\hline & Subtalar & $0.05 \pm 0.05$ & $0.56 \pm 0.46$ \\
\hline
\end{tabular}




\subsubsection{Video}

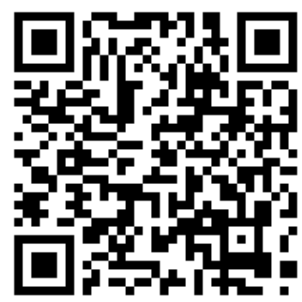

Real-time EMG-driven modelling: Jump Task. Video demonstrating the real-time EMG-driven framework working in a gait lab. The framework connect via TCP/IP to the motion capture system and the EMG amplifier. The resulting torque and muscles force are then show in real-time in a user graphical interface (GUI).

https://www.youtube.com/watch?time continue $=1 \& v=y X A T F 7 P 216 E \& f e a t u r e$ $\equiv$ emb $\log \mathrm{O}$

\subsection{Appendix to Chapter 3}

\subsubsection{Video}

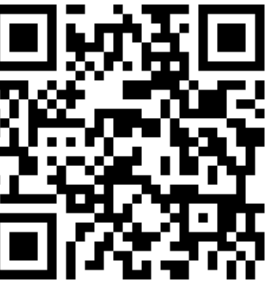

A real-time HMI for the estimation of neuromuscular states during human-exoskeleton. This video presents a realtime EMG-driven musculoskeletal model for the control of a bilateral ankle exoskeleton. Experiments were realized on one subject wearing the Achilles ankle exoskeleton with Minimal impedance and with motor off. Motor tasks included treadmill walking at $2 \mathrm{~km} / \mathrm{h}$ and $3 \mathrm{~km} / \mathrm{h}$ and transition between walking and stair ascending. We show that this HMI can process and give information on joint torque and internal muscle parameter on multiple motor tasks involving hums-exoskeleton interaction.

\section{https://www.youtube.com/watch?v=IVHFi9ui72U}




\subsection{Appendix to Chapter 4}

\subsubsection{Supplementary Figures}
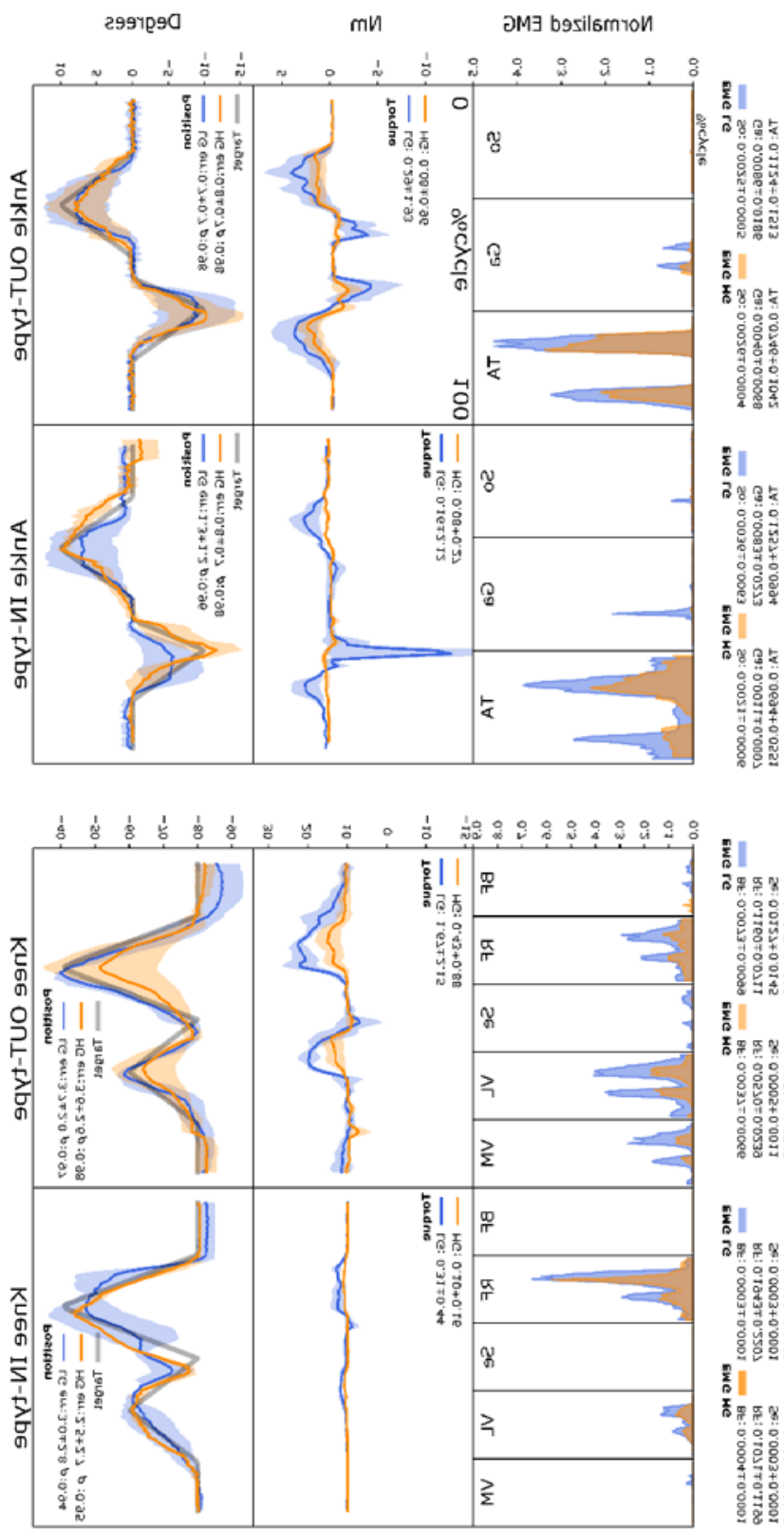

fิ घี

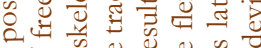
峲

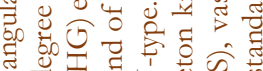
过它造的

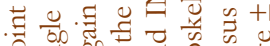
응

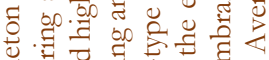

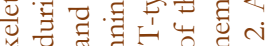

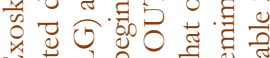

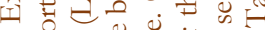
ल पे बे 0

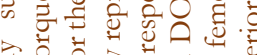

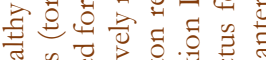

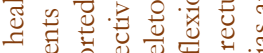

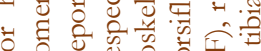

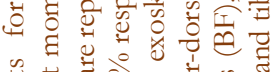

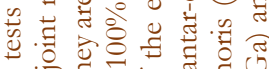

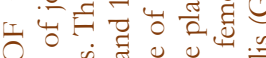

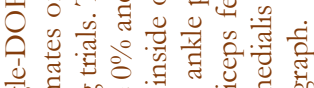
ह1

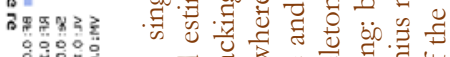

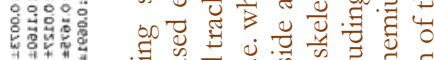

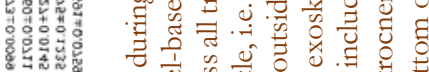

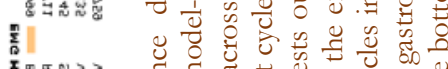

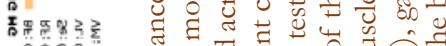

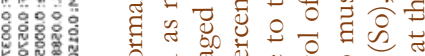

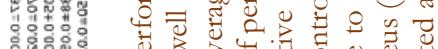

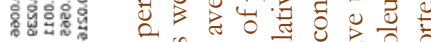

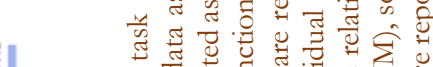

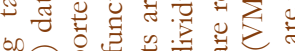

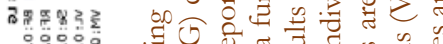

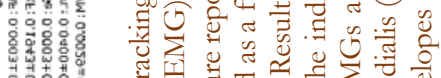

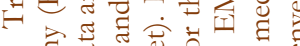

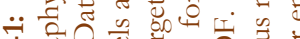
$\frac{n}{\frac{n}{2}}$

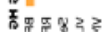

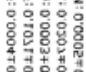

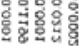

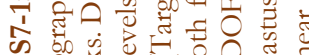
ง ป

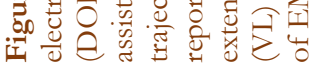




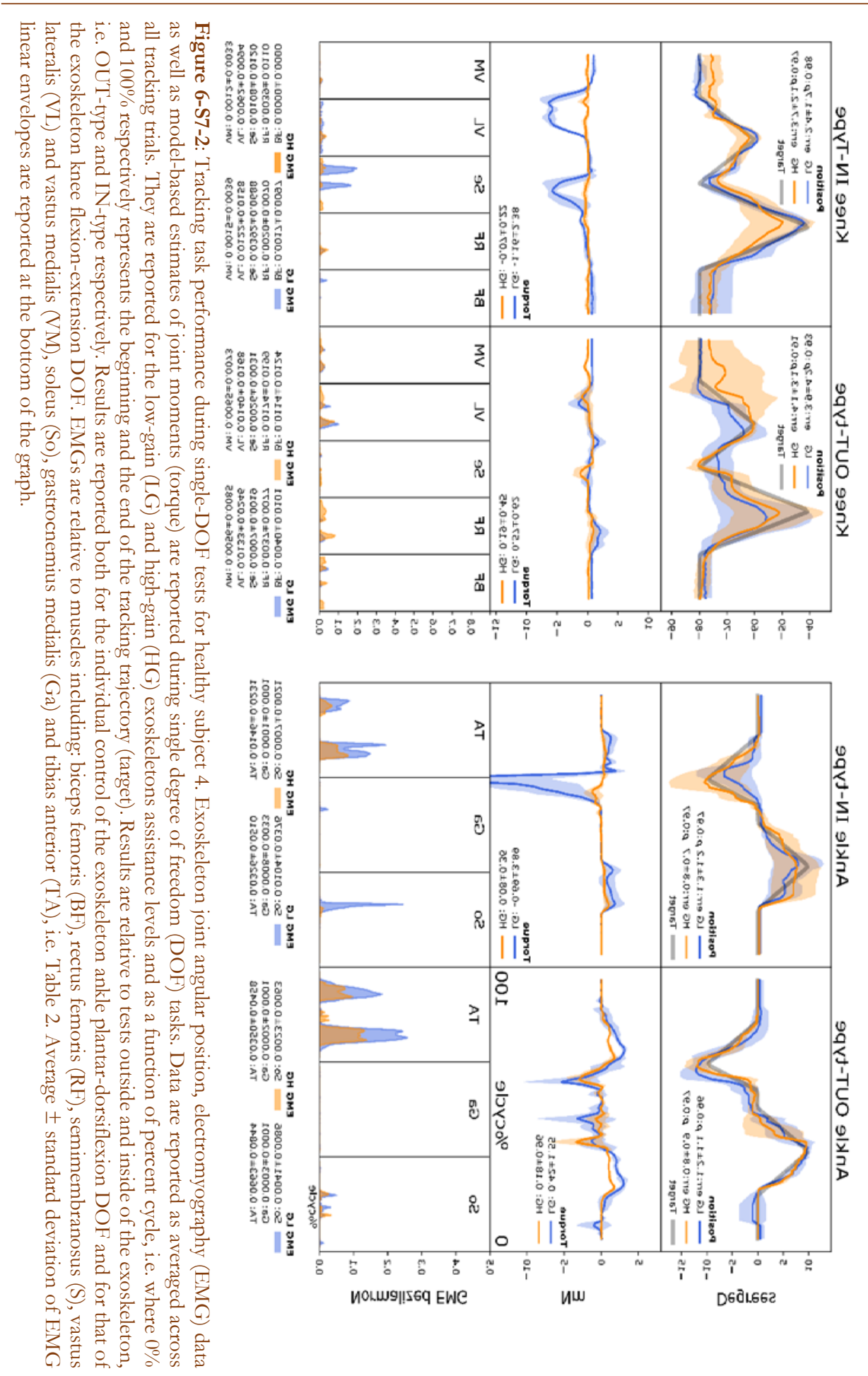




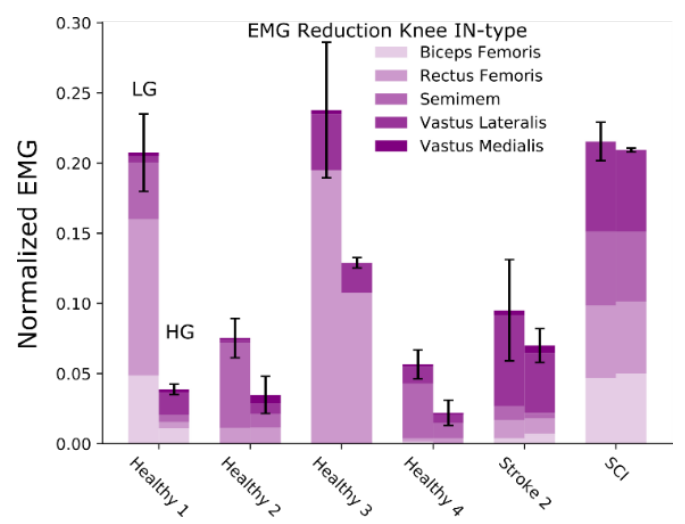

Figure 6-S7-3: EMG amplitude modulation between exoskeleton low- and high-assistance levels during single knee flexion-extension, IN-type experiments. Electromyography (EMG) amplitude is consistently reduced when transitioning from low-gain (LG, left-hand vertical bar) to high-gain (HG, right-hand vertical bar) exoskeleton support levels. Experiments were performed while wearing the robotic exoskeleton, i.e. IN-type tests. For each subject (Healthy 1-4) as well as for stroke patient 2 (Stroke) and the spinal cord injury (SCI) patient (Table 1) the vertical bars report mean normalized EMG amplitude stacked vertically for each muscles along with standard deviation (i.e. see black vertical lines).

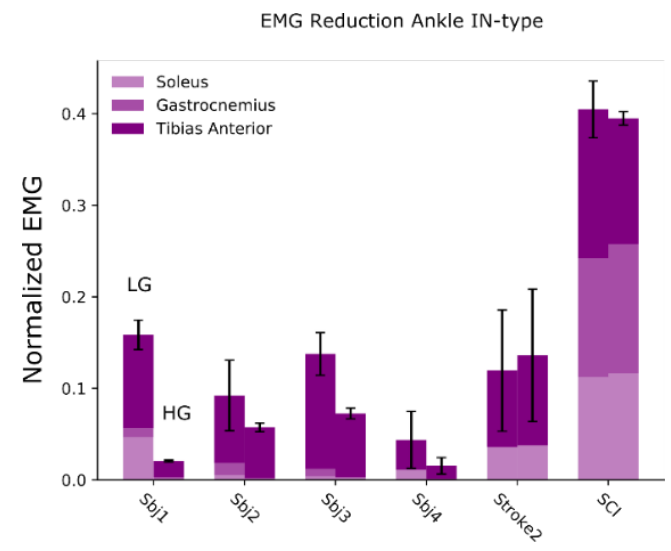

Figure 6-S7-4: EMG amplitude modulation between exoskeleton low- and high-assistance levels during single ankle plantar-dorsi flexion, IN-type experiments. Electromyography (EMG) amplitude is consistently reduced when transitioning from low-gain (LG, left-hand vertical bar) to high-gain (HG, right-hand vertical bar) exoskeleton support levels. Experiments were performed while wearing the robotic exoskeleton, i.e. IN-type tests. For each subject (Healthy 1-4) as well as for stroke patient 2 (Stroke) and the spinal cord injury (SCI) patient (Table 1) the vertical bars report mean normalised EMG amplitude stacked vertically for each muscles along with standard deviation (i.e. see black vertical lines). 


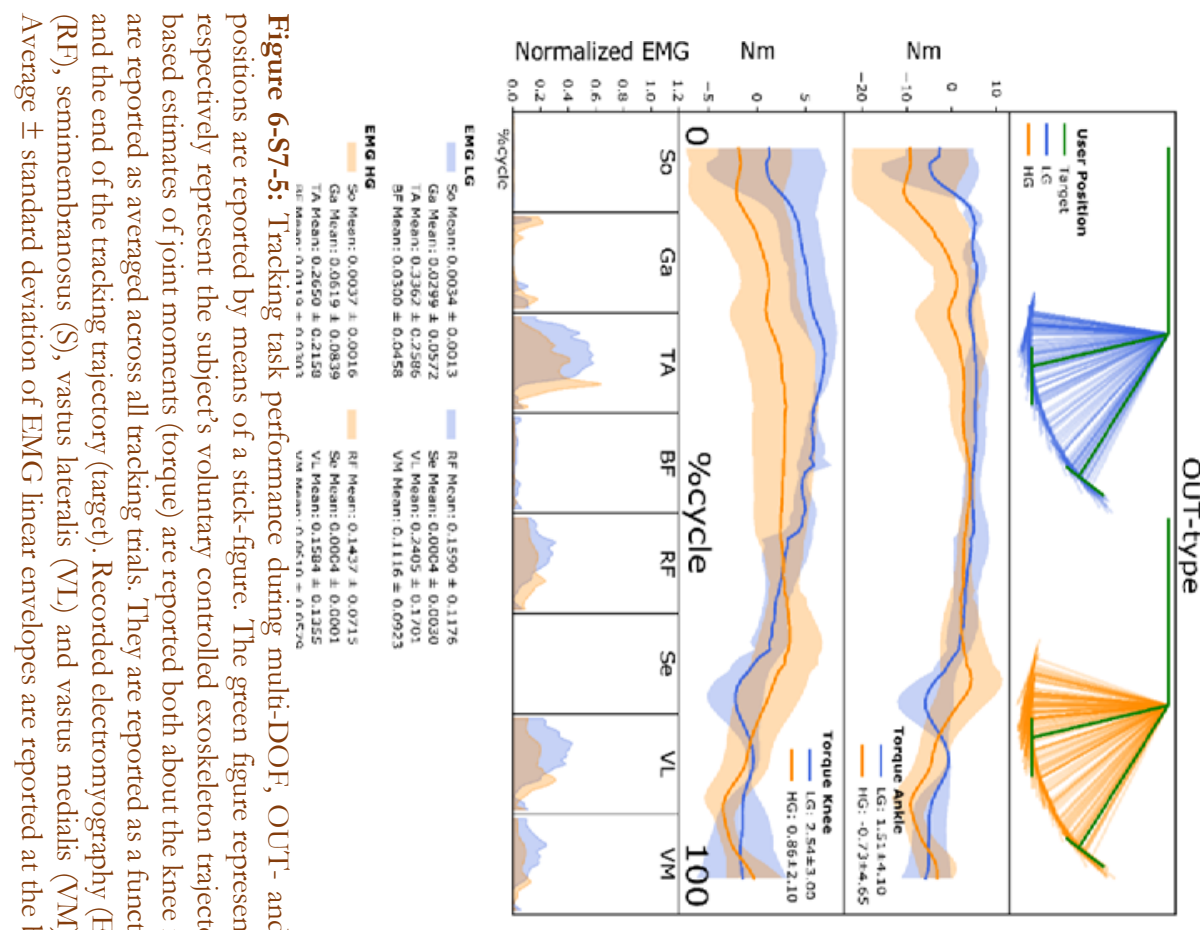



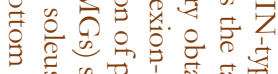

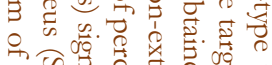

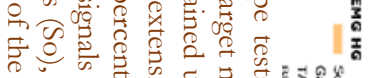

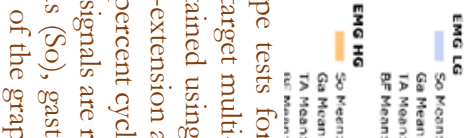

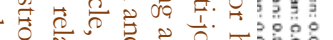

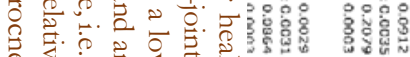

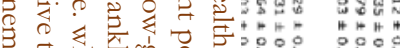

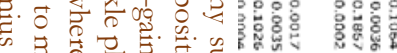

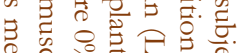

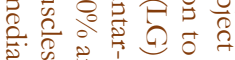

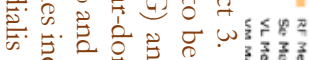

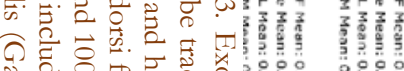

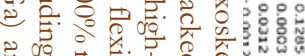

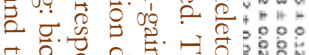

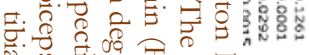

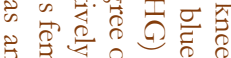

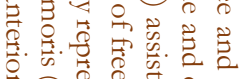

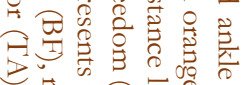

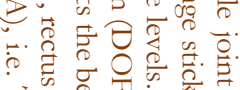

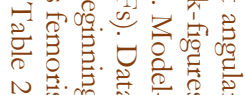

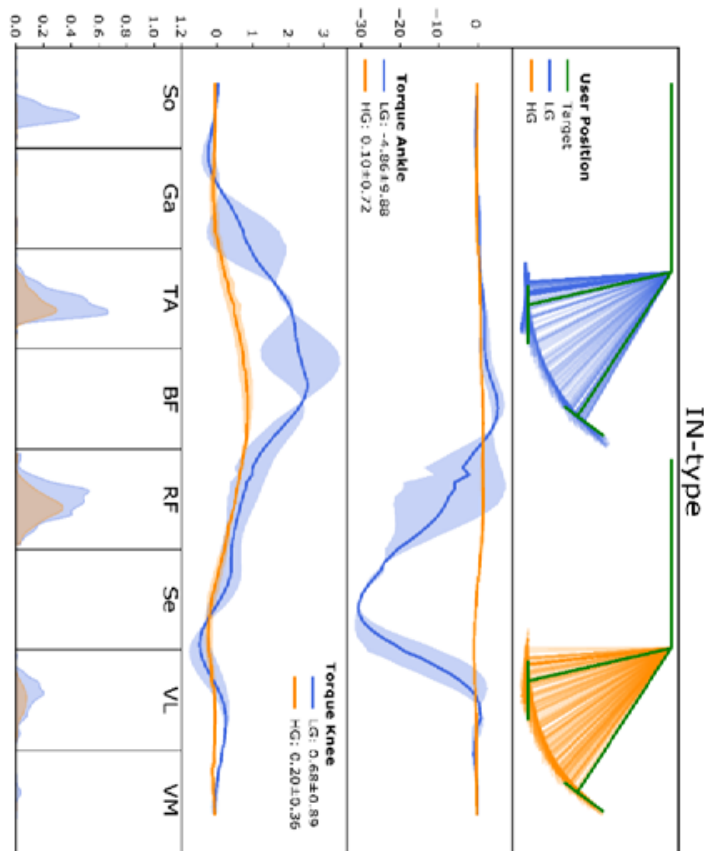



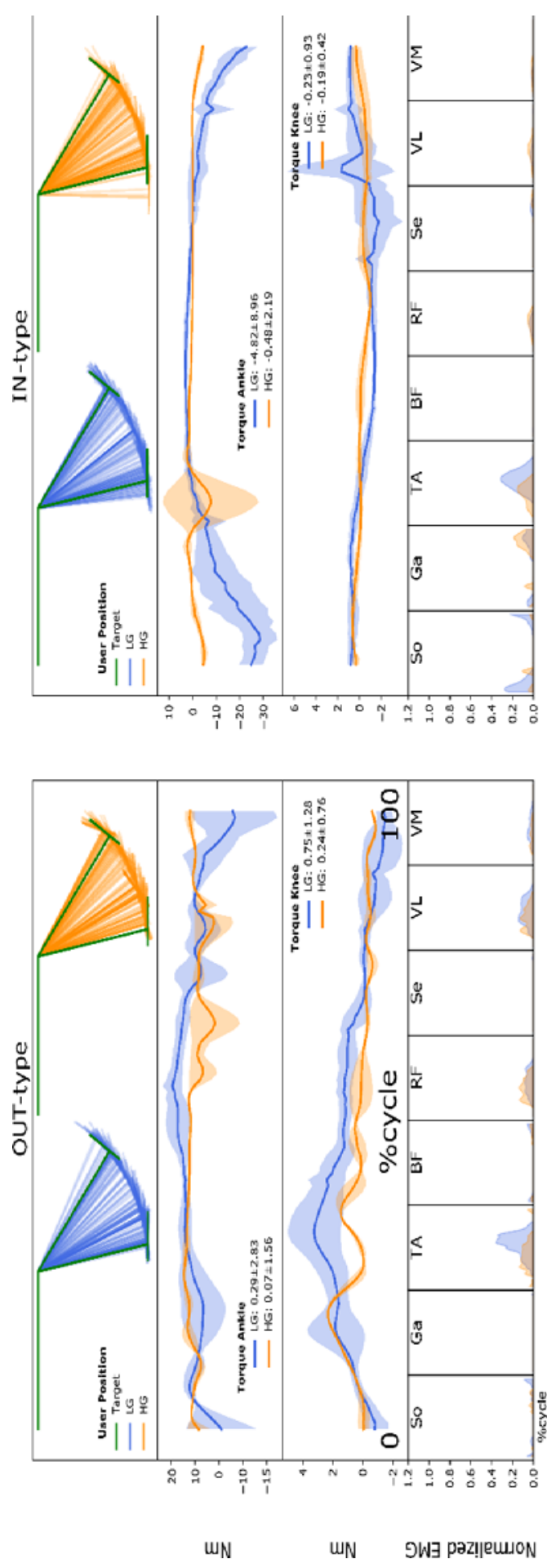

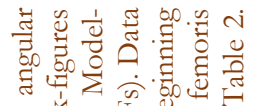
. o

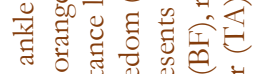
च्च च्च

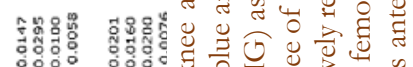

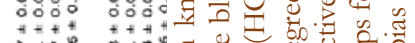

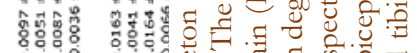

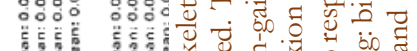

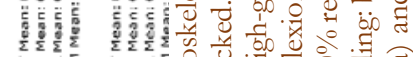

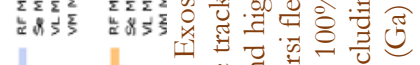

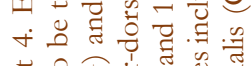

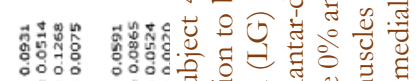

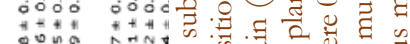

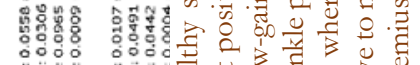

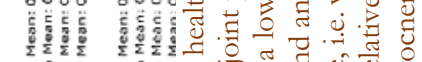

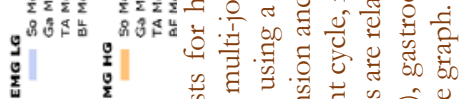

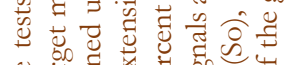

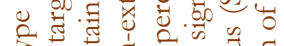

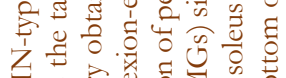

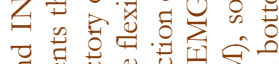

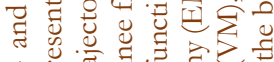

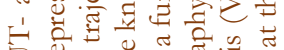

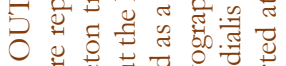

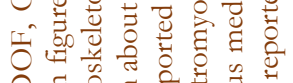
I

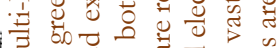

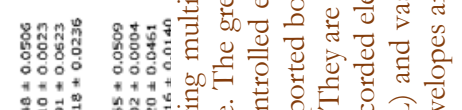

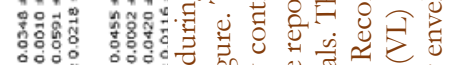

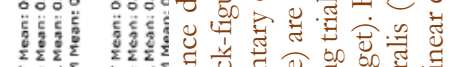

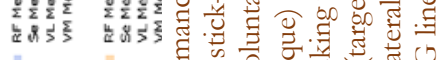

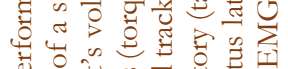

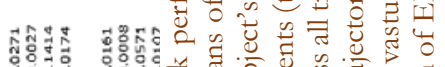

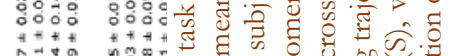

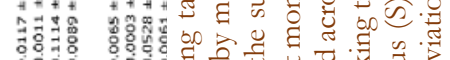
管

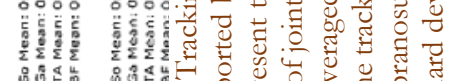

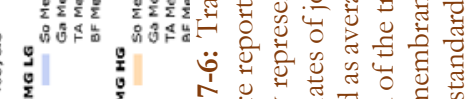

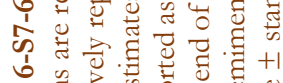

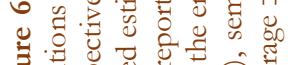

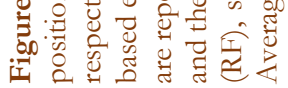




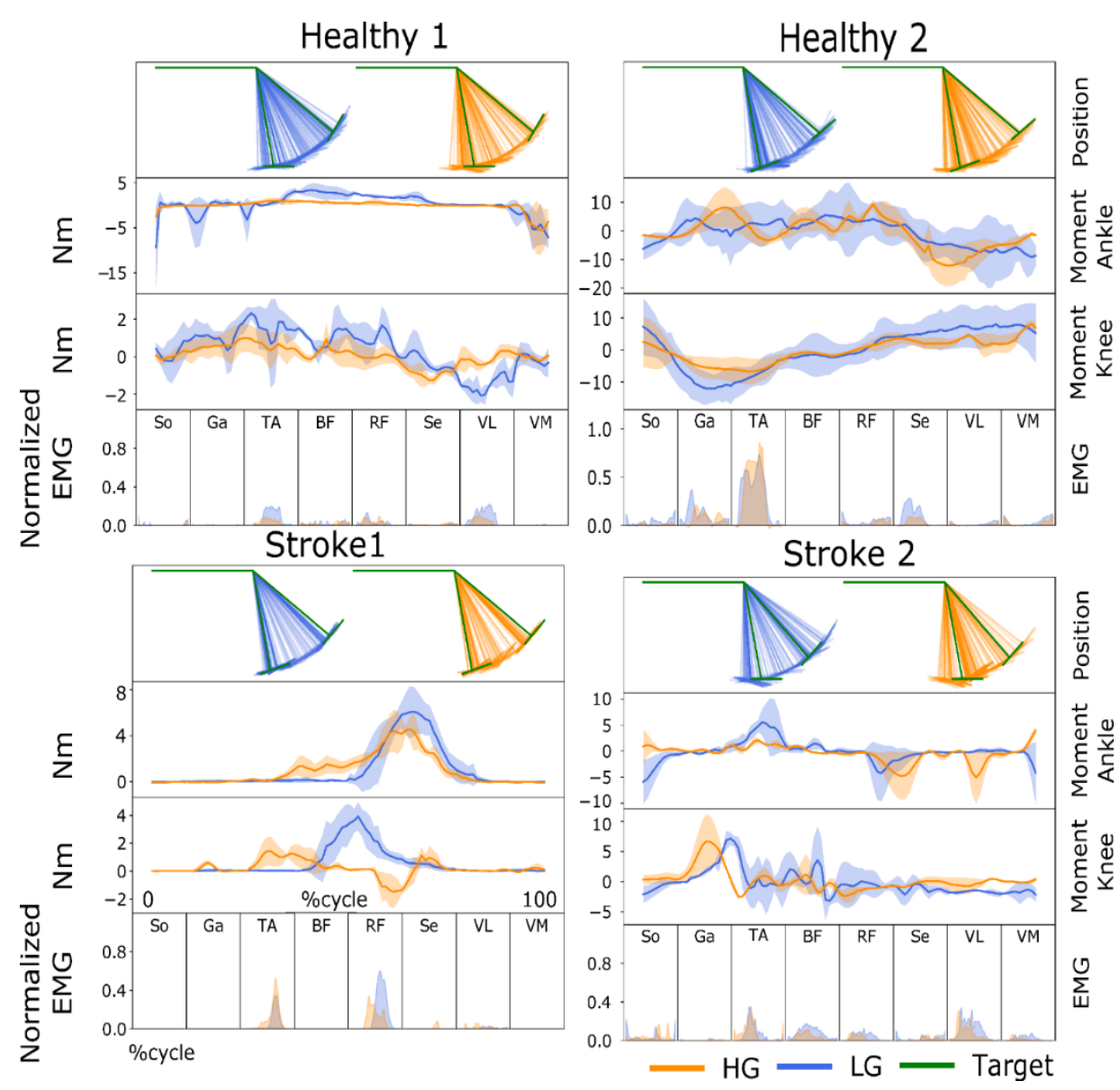

Figure 6-S7-7: Tracking task performance during multi-DOF, OUT-type tests. Exoskeleton knee and ankle joint angular positions are reported by means of a stick-figure. The green figure represents the target multi-joint position to be tracked. The blue and orange stick-figures respectively represent the subject's voluntarily controlled exoskeleton trajectory obtained using a low-gain (LG) and high-gain (HG) assistance levels. Model-based estimates of joint moments are reported both about the knee flexion-extension and ankle plantar-dorsi flexion degree of freedom (DOFs). Data are reported as averaged across all tracking trials. They are reported as a function of percent cycle, i.e. where $0 \%$ and $100 \%$ respectively represents the beginning and the end of the tracking trajectory (Target). Results are relative to tests inside of the exoskeleton, i.e. OUT-type. Data are reported for two representative healthy subjects (Healthy 1-2) and two stroke patients (Stroke 1-2), i.e. Table 1. Recorded electromyography (EMGs) signals are relative to muscles including: biceps femoris $(\mathrm{BF})$, rectus femoris $(\mathrm{RF})$, semimembranosus $(\mathrm{S})$, vastus lateralis $(\mathrm{VL})$ and vastus medialis $(\mathrm{VM})$, soleus (So), gastrocnemius medialis $(\mathrm{Ga})$ and tibialis anterior (TA), i.e. Table 2 . 


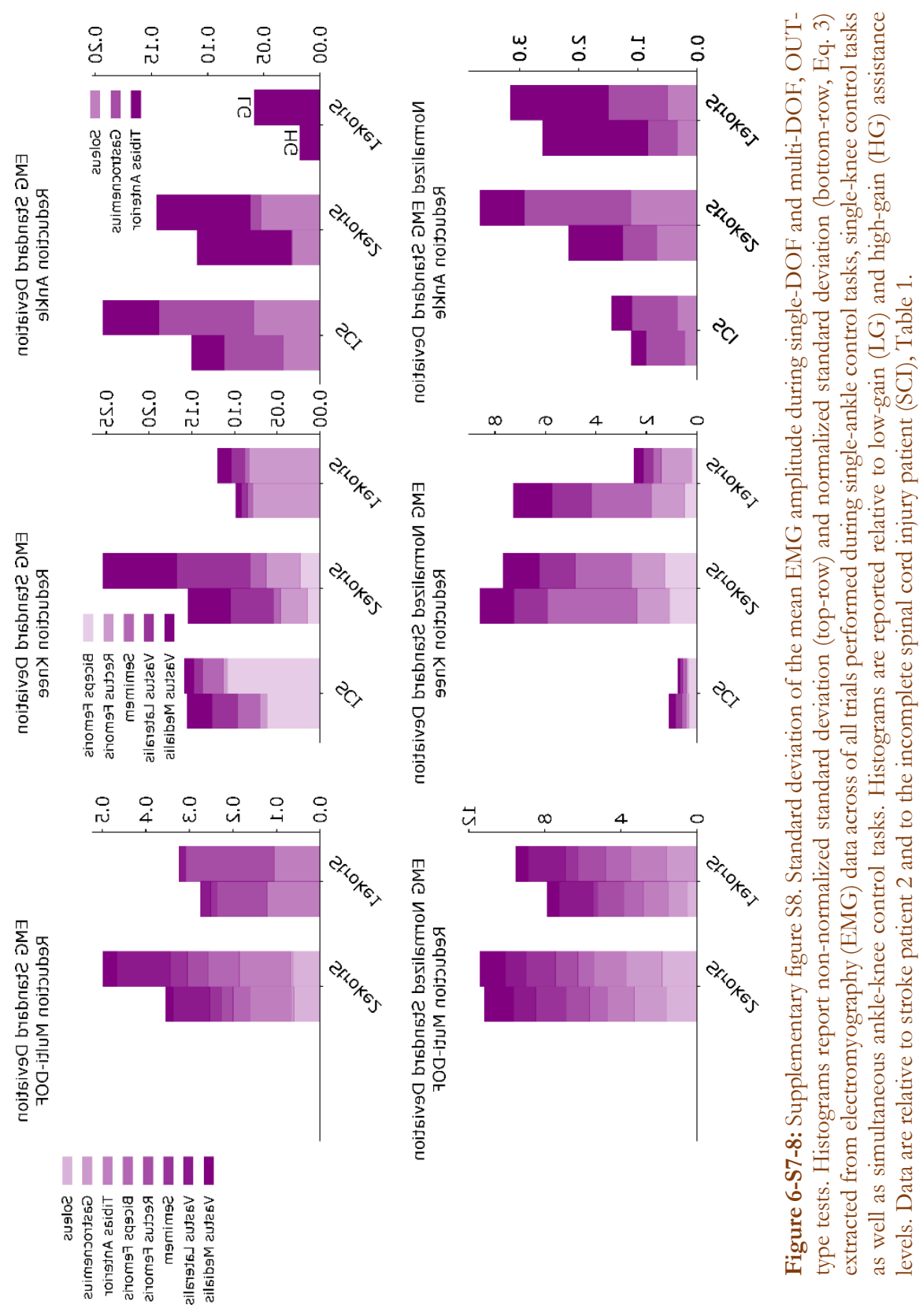



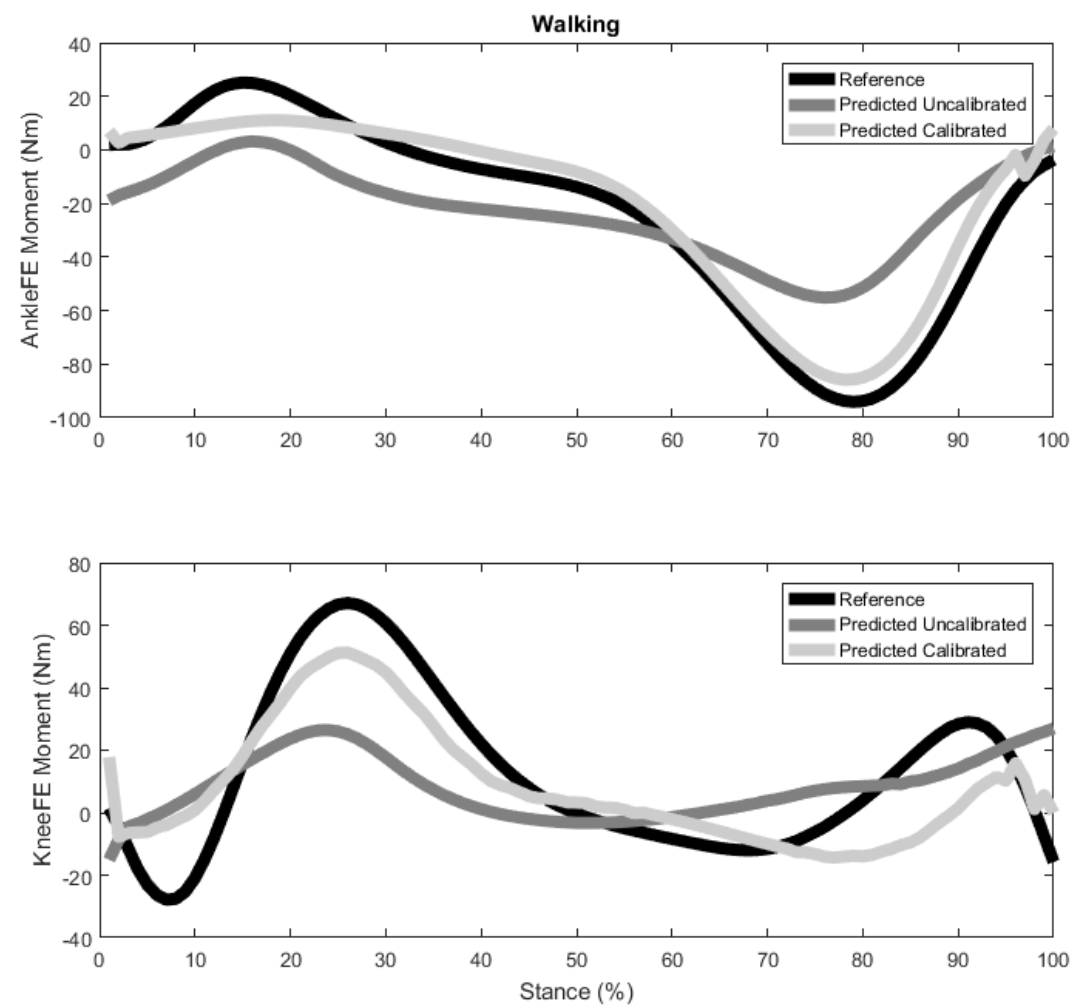

Figure 6-S7-9: Predicted moment for the Ankle plantar-dorsiflexion and the Knee flexion-extension using an uncalibrated model. Average of 5 subjects for a locomotion task (fast walking), with in grey line the predicted moment using an uncalibrated model, in light grey the predicted moment using a calibrated model and in black line the experimental moment from inverse dynamics using the OpenSim Software. Data were taken from (1). 


\subsubsection{Supplementary Tables}

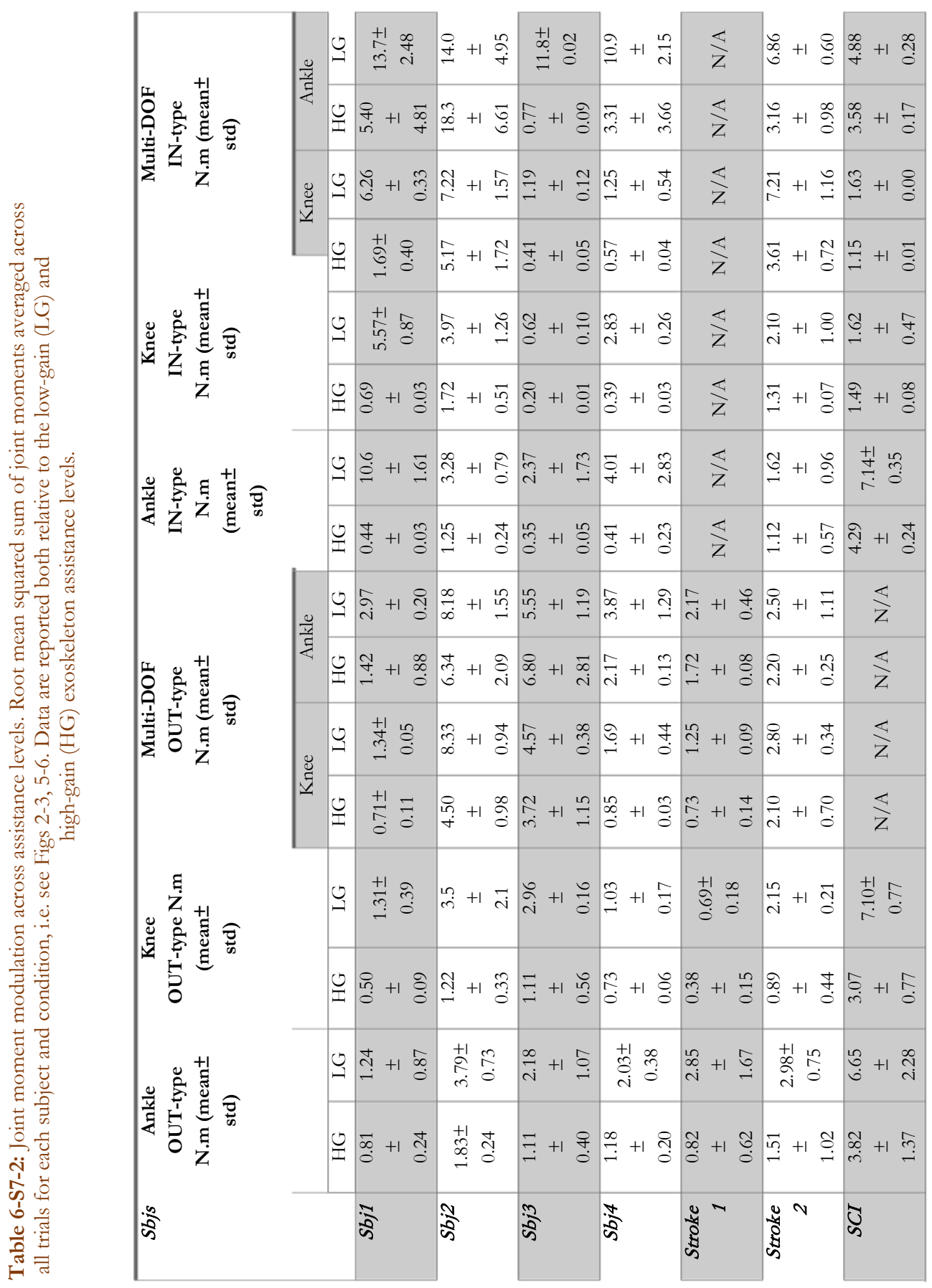




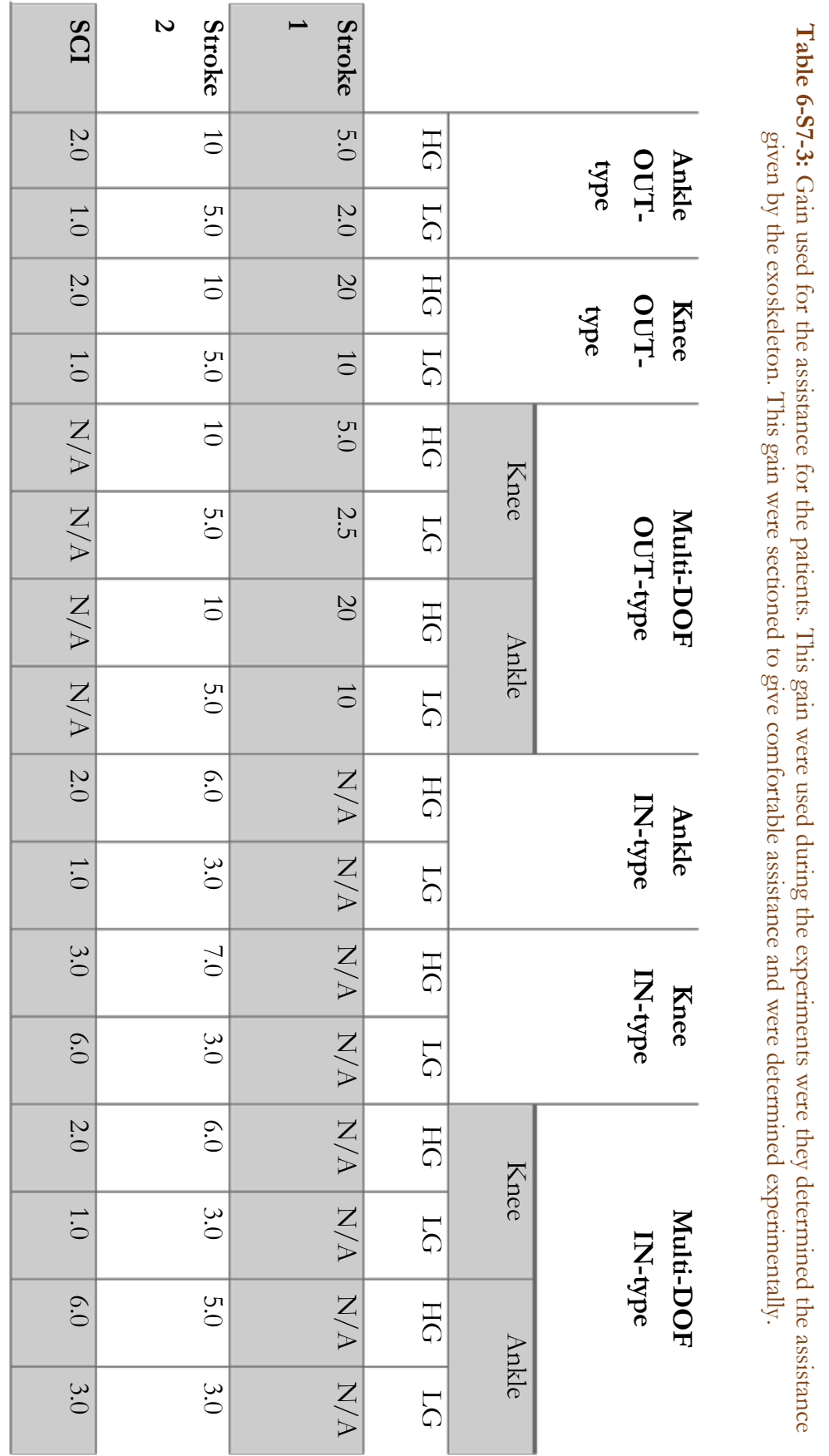




\subsubsection{Video}

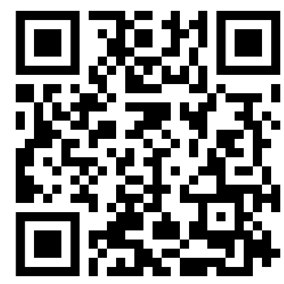

Voluntary control of wearable robotic exoskeletons by patients with paresis. Video presenting the real-time control of the $\mathrm{H} 2$ exoskeleton by a stroke patient. The video also presents the GUI showed to the user and the voluntary control of the exoskeleton with the patient inside and outside of the exoskeleton.

\section{https://www.youtube.com/watch?v=5 vsu1pHoNs}

\subsection{Appendix to chapter 5}

\subsubsection{Video}

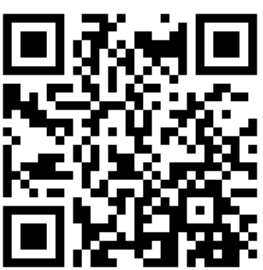

Voluntary and Continuous Control of Robotic Exoskeletons during Locomotion. In this video, we present our experiments conducted with the WR2 exoskeleton on locomotion. We show the exoskeleton giving assistance based on the neuromusculoskeletal model's computed ankle joint torque for locomotion at $1.8 \mathrm{~km} / \mathrm{h}$ and with no inclination.

https:/ www.youtube.com/watch?v=JlzlpvC1xzo 
Section: Appendix 


\section{ACKNOWLEDGEMENTS}

I would like first to acknowledge my family, in particular my parents for their moral and financial support over the whole span of my study. I would like to also especially thanks to my grandma for her delicious cooking and positive attitude. Merci Maman, Papa et Mamie. I would like to also thank my wife, Lupita (Maria Guadalupe) for her love and taking care of me at home. Her Mexican cooking helped me a lot to go through the last year of my $\mathrm{PhD}$. Gracias Lupita.

I want to also thank Massimo to offer me the possibility to pursue a $\mathrm{PhD}$ and for his support, scientific and moral. His guidance during my $\mathrm{PhD}$ and before it helped me to be a better scientist and start my career on the right track. I am also very thankful to him for offering me to follow him at the University of Twente. Grazie Massimo.

I would like to also thank my current and previous promotor Herman and Dario to have accepted me in their lab in Enschede and Göttingen.

I would also like to thank my friends and colleagues from Enschede. First, I want to thanks my officemate Ronald (the stat guy), Simone (the dutch translator), Huawei and René (the bike guy).

I also want to thank the Spanish/Mexican cartel Cristina (la Jeffa), Alejandro, Pablo, Rafael, Antonio, and Ander. As well as the one adopted by the cartel Edwin, Donatella and Michelle. $\mathrm{My} \mathrm{PhD}$ will have been less fun without them. I want to thank also the Vici boy Wolfgang, Kyrian, Quint and Allan. Special thanks to the WR2 reparation crew Quint, Wolfgang, Gijs and Michiel. I also want to thanks our secretary trio: Lianne, Jeanine and Yolanda as well as my friends from Enschede Utku, Fusun, Roos and Aisen. Finally, I want to thanks the ET-BE crew: Nikolai, Kostas, Arvid, Wouter, Marco, Ali, Bart, Nauzef, Manav and the Robotics surgical lab members. I want to give a special thanks to Allan and Huawei for checking my dissertation.

I would like to thank also my friends and colleagues from Göttingen: The Serbian team Marko, Ivan and Strahinja, the

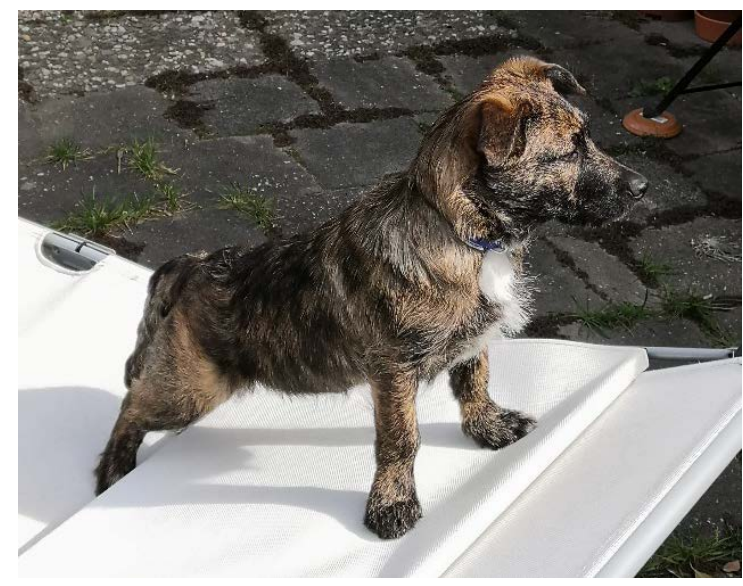


Italian team Silvia, Margherita, Alessandro, Leonardo, Giacomo, Antonietta and Francesco and the lone wolf Spanish Andres.

I would also like to give a special thanks to the cutest dog Caxcan (see picture for cuteness). 